\title{
Solar Stereoscopy and Tomography
}

\author{
Markus J. Aschwanden \\ Solar and Astrophysics Laboratory, \\ Lockheed Martin, Advanced Technology Center, \\ Bldg. 252, Org. ADBS, 3251 Hanover St., \\ Palo Alto, CA 94304, USA \\ email: aschwanden@lmsal.com \\ http://www.lmsal.com/ aschwand/
}

Accepted on 30 August 2011

Published on 10 October 2011

\begin{abstract}
We review stereoscopic and tomographic methods used in the solar corona, including ground-based and space-based measurements, using solar rotation or multiple spacecraft vantage points, in particular from the STEREO mission during 2007-2010. Stereoscopic and tomographic observations in the solar corona include large-scale structures, streamers, active regions, coronal loops, loop oscillations, acoustic waves in loops, erupting filaments and prominences, bright points, jets, plumes, flares, CME source regions, and CME-triggered global coronal waves. Applications in the solar interior (helioseismic tomography) and reconstruction and tracking of CMEs from the outer corona and into the heliosphere (interplanetary CMEs) are not included.
\end{abstract}

This review is licensed under a Creative Commons Attribution-Non-Commercial-NoDerivs 3.0 Germany License. http://creativecommons.org/licenses/by-nc-nd/3.0/de/ 


\section{Imprint / Terms of Use}

Living Reviews in Solar Physics is a peer reviewed open access journal published by the Max Planck Institute for Solar System Research, Max-Planck-Str. 2, 37191 Katlenburg-Lindau, Germany. ISSN 1614-4961.

This review is licensed under a Creative Commons Attribution-Non-Commercial-NoDerivs 3.0 Germany License: http://creativecommons.org/licenses/by-nc-nd/3.0/de/

Because a Living Reviews article can evolve over time, we recommend to cite the article as follows:

Markus J. Aschwanden, "Solar Stereoscopy and Tomography",

Living Rev. Solar Phys., 8, (2011), 5. [Online Article]: cited [<date $>$ ], http://www.livingreviews.org/lrsp-2011-5

The date given as $<$ date $>$ then uniquely identifies the version of the article you are referring to.

\section{Article Revisions}

Living Reviews supports two ways of keeping its articles up-to-date:

Fast-track revision A fast-track revision provides the author with the opportunity to add short notices of current research results, trends and developments, or important publications to the article. A fast-track revision is refereed by the responsible subject editor. If an article has undergone a fast-track revision, a summary of changes will be listed here.

Major update A major update will include substantial changes and additions and is subject to full external refereeing. It is published with a new publication number.

For detailed documentation of an article's evolution, please refer to the history document of the article's online version at http: //www. livingreviews.org/lrsp-2011-5. 


\section{Contents}

1 Introduction $\quad 5$

2 History $\quad 6$

2.1 Ground-based stereoscopy and tomography . . . . . . . . . . . . . . . . . . . 6

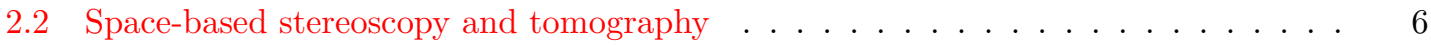

2.3 The STEREO mission . . . . . . . . . . . . . . . . . . . 7

3 Stereoscopy and Tomography Methods $\quad 9$

3.1 Solar-rotation stereoscopy . . . . . . . . . . . . . . . . . . . . . 9 9

3.1.1 Static solar-rotation stereosocopy . . . . . . . . . . . . . . . 9 9

3.1.2 Dynamic solar-rotation stereosocopy . . . . . . . . . . . . . . 11

3.2 Solar-rotation tomography . . . . . . . . . . . . . . . . . . . 11

3.2 .1 EUV and Soft X-rays . . . . . . . . . . . . . . . . 11

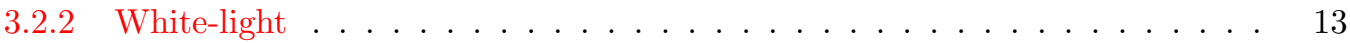

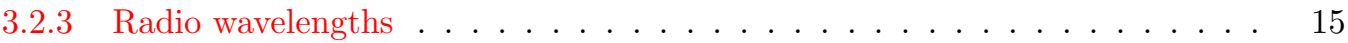

3.3 Stereoscopic triangulation or tie-point method . . . . . . . . . . . . . . . 19

3.4 Magnetic stereoscopy . . . . . . . . . . . . . . . . . . . . . . . 22

3.5 Stereoscopic tomography and 3D forward-fitting . . . . . . . . . . . . . 25

4 Stereoscopic and Tomographic Observations $\quad 28$

4.13 D reconstruction of the large-scale corona . . . . . . . . . . . . . . . . . 28

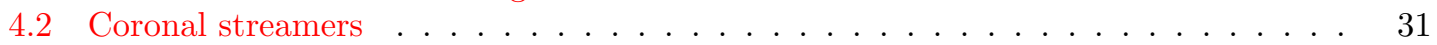

4.3 Active regions . . . . . . . . . . . . . . . . . . . . . . . . . . . . . . . . . . . . . . . . . . . . . . . . . . . . . . .

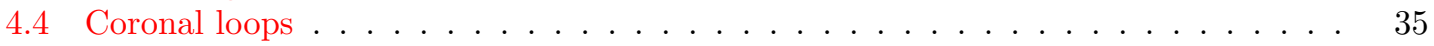

4.4.1 Hydrostatic scale height of loops . . . . . . . . . . . . . . . . . . . . . . . . . . . . . . . . . . . .

4.4 .2 Hydrodynamics of loops . . . . . . . . . . . . . . . . . . . . 40

4.4.3 Magnetic fields of loops . . . . . . . . . . . . . . . . . . . 43

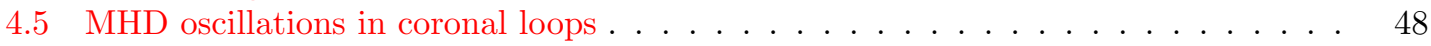

4.6 MHD waves in coronal loops . . . . . . . . . . . . . . . . . . . . . . . . . . . . . . . . . . . . . . . .

4.7 Quiescent filaments and prominences . . . . . . . . . . . . . . . . 58

4.8 Erupting filaments and prominences . . . . . . . . . . . . . . . . . 59

4.9 Bright points, jets, and plumes . . . . . . . . . . . . . . . . . . . . . . . . . . . . . . . . . .

4.10 Solar flares . . . . . . . . . . . . . . . . . . . . . . . . . . . . . . . . . . . . . . 65

4.11 CME source regions and EUV dimming . . . . . . . . . . . . . . . . . . . . . . . . . . . . . . . . . . . . . .

4.12 Global coronal waves . . . . . . . . . . . . . . . . . . . . . . . 71

5 Summary $\quad 74$

$\begin{array}{llr}6 & \text { Acknowledgements } & 77\end{array}$

$\begin{array}{lr}\text { References } & 78\end{array}$

\section{List of Tables}

$1 \quad$ Misalignment statistics of stereoscopically triangulated loops in four active regions. 46

2 Flare and CME events with loop oscillations and waves observed with STEREO/EUVI. 49 



\section{Introduction}

Stereoscopy (from Greek stereos = solid body and skopein = to see) includes a variety of methods to enable or enhance the depth perception that leads to a three-dimensional (3D) view of an object. Our eyes process stereoscopic information automatically when our brain correlates the visual inputs from the left and right eye, at near distances. Astronomical images, however, contain only two-dimensional (2D) brightness distributions, but can produce stereoscopic 3D information from two different aspect angles. Just by taking pictures of the night sky six months apart, we have a baseline change of two astronomical units (AU) and can triangulate the distance to the nearest stars that show a stereoscopic parallax with respect to the distant stars with no parallax. Here we are concerned with solar imaging, which can be stereoscopically processed by using either the solar rotation to change the aspect angle $\left(\approx 13^{\circ}\right.$ per day $)$ or multiple spacecraft that are spaced some significant fraction of $1 \mathrm{AU}$ apart. The prime solar-dedicated space mission that is designed to exploit stereoscopic information to the fullest is the Solar TErrestrial RElations Observatory (STEREO) mission, launched in 2006. Solar stereoscopy became an important part of 3D geometric and physical modeling of solar dynamic phenomena, but still presents formidable challenges to construct detailed 3D models from two (or three) aspect angles only.

Tomography (from Greek tomos = cut, slice and graphein = to write) is a related 3D reconstruction method that uses images (or slices) from many aspect angles. Practically, tomography is accomplished by 2D imaging of different sections through a penetrating (X-ray or radio) wave, used in medical radiology, archeology, biology, geophysics, oceanography, material science, and astrophysics. Applications in solar physics include 3D reconstruction of the optically-thin plasma in the solar corona or in coronal mass ejections, using soft X-ray and extreme-ultraviolet (EUV) imagers or white-light coronagraphs. The solar-dedicated STEREO mission carries a set of coronagraphs onboard that can map the coronal plasma from two different directions, which represents a kind of minimum tomography. Alternatively, the solar rotation can be used to provide many slices from different viewing angles, but tomographic reconstruction is then challenged by dynamical changes during the observing period. 3D reconstructions inside the Sun (helioseismic tomography) or in the heliosphere, such as interplanetary coronal mass ejections (CMEs) or the solar wind, are not covered here.

In the following review we cover first the history of solar stereoscopy, which includes solarrotation based stereoscopy, single- and multi-spacecraft stereoscopy in the pre-STEREO era, as well as a brief description of the STEREO mission. In Section 3 we describe the stereoscopic and tomographic methods that have been developed and applied to solar data. In Section 4 we provide applications of the methods described in Section 3, i.e., a systematic description of stereoscopic and tomographic observations of phenomena in the solar corona (active regions, flares, filaments, prominences, CME source regions, CME-triggered global waves). In the final Section 5 we summarize the main scientific results that were or could be obtained from stereoscopic and tomographic data analysis.

There exists no comprehensive review on STEREO results at this time, but STEREO-dedicated Topical Volumes have been published in the following four journal issues: Space Science Reviews Vol. 136 (2008), Solar Physics Vol. 256 and 259 (2009), and Journal of Atmospheric and SolarTerrestrial Physics Vol. 73 (2011; Srivastava et al., 2011). Some minor reviews that cover partial aspects of solar stereoscopy include coronal regions (Wiegelmann et al., 2009; Aschwanden and Wülser, 2011), heliospheric coronal mass ejections (Howard and Tappin, 2009a,b; Tappin and Howard, 2009; Harrison et al., 2009; Mierla et al., 2010), theoretical modeling (Khodachenko and Rucker, 2005; Aschwanden et al., 2008a), and short STEREO mission summaries (Schmidt and Bothmer, 1996; Davila, 1998; Kaiser, 2005; Staedter, 2006; Matthews and Culhane, 2007; Kaiser et al., 2008; Brown, 2009). 
Markus J. Aschwanden

\section{History}

The history of solar stereoscopy and tomography is fairly short, because ground-based methods using the solar rotation are very limited, while space-based imagers that travel to interplanetary distances are very few and not solar-dedicated in the pre-STEREO era. And finally, the first (and only) solar-dedicated mission with stereoscopic capabilities, STEREO, is less than five years old at the time of writing.

\subsection{Ground-based stereoscopy and tomography}

Ground-based solar observations are limited to optical and radio wavelengths. Since there is no appreciable parallax effect on global distances, stereoscopic or tomographic methods can only be carried out by taking advantage of the solar rotation. However, because of the huge brightness contrast, the solar corona can only be imaged during total eclipses or using (disk-occulting) coronagraphs.

The first attempts of a tomographic reconstruction of the 3D density distribution of the solar corona, based on a daily series of ground-based coronagraph images and potential-field extrapolations from magnetograms, is described in Altschuler (1979), which shows multiple coronal streamers as viewed from above the solar north pole.

A first stereoscopic triangulation of ray-like coronal structures at $\approx 1.2-3.0$ solar radii was achieved from two white-light pictures during the total solar eclipse of 1991 July 11, observed 3 hours apart from sites at Hawaii, Mexico, and Brazil (Koutchmy and Molodenskii, 1992), which yields a parallax angle of $\approx 1.6^{\circ}$. Using similar white-light images taken with a radial filter from another eclipse, the 3D density structure of streamers extending into heliospheric layers was reconstructed by Koutchmy et al. (1997) and Vedenov et al. (2000), visualized as stereoscopic image pairs.

High-resolution solar images in radio wavelengths became readily available with radio interferometers such as the Very Large Array (VLA) at frequencies of $\gtrsim 1 \mathrm{GHz}$ around 1980. Reasonably stable radio-emitting structures in the solar corona, such as the optically-thick layers of free-free emission in active regions or the gyro-resonance layers above sunspots, can be imaged day by day, which allows to measure the altitudes from the parallax effect of the radio source centroids. Synthesizing these altitude measurements at multiple radio frequencies allows then to construct a 3D model of the magnetic field and/or plasma density, which is called the method of radio stereoscopy (Aschwanden et al., 1992; Aschwanden and Bastian, 1994a,b; Aschwanden et al., 1995).

Another approach to use radio-emitting coronal sources for coronal 3D density reconstruction is frequency tomography, which was pioneered at the Russian RATAN radio interferometer (Bogod and Grebinskij, 1997; Gelfreikh, 1998; Grebinskij et al., 2000). However, the multi-frequency imaging maps of optically thin plasma require a free-free opacity model to convert the radio frequencies into a geometric altitude.

\subsection{Space-based stereoscopy and tomography}

Helios 1 and Helios 2 were the first solar-observing spacecraft (1974-1980) that were sent as close as $0.3 \mathrm{AU}$ to the Sun and, thus, provided a stereoscopic vantage point. A first 3D reconstruction of a coronal mass ejection (CME) was accomplished with a computer assisted tomography (CAT) technique by Jackson and Froehling (1995), using the zodiacal light photometers on the spacecraft Helios 2 (0.3 AU from the Sun) and white-light coronagraph images from Solwind (near Earth).

After the launch of Skylab in 1973, a number of solar-dedicated space missions came along with imaging capabilities in extreme ultraviolet and soft X-rays (SMM, Yohkoh, SOHO), besides whitelight coronagraphs (onboard SMM and SoHO). However, all these solar-dedicated space missions

Living Reviews in Solar Physics

http://www. livingreviews.org/lrsp-2011-5 
had near-Earth orbits, so that no true stereoscopy was feasible, but solar rotation-based stereoscopy could be carried out for reasonably stable structures in the solar corona. Such rotation-based stereoscopy was applied to coronal loops using Skylab images (Berton and Sakurai, 1985), to produce quasi-stereoscopic images of the soft X-ray-emitting corona using Yohkoh images (Batchelor, 1994), to perform 3D tomography of a coronal hole using a 2-week's worth of Yohkoh images (Hurlburt et al., 1994), or to reconstruct the 3D geometry of prominences from synoptic SOHO/EIT images (Foullon, 2003). Since the plasma dynamics in individual coronal loops is relatively fast (hours) compared with the required time interval to measure a significant parallax effect (about a day), while the confining magnetic field is changing on a longer time scale, a special dynamic stereoscopy method has been developed to determine the 3D geometry of planar loops using SOHO/EIT images (Aschwanden et al., 1999, 2000).

The LASCO white-light coronagraph onboard SOHO was especially designed to detect and track CMEs. A tomographic inversion method was developed by Quémerais and Lamy (2002) to invert the 2D line-of-sight integrated electron density distribution in the solar corona from a single spacecraft (LASCO-C2), using additional constraints of spherical or axial symmetry. Another tomographic method was developed by Moran and Davila (2004), Moran et al. (2010), and Dere et al. (2005) to reconstruct the 3D density distribution from the combination of intensity and polarized brightness images from LASCO. Combining LASCO images over a 14-day period, Frazin et al. (2007) accomplished a 3D tomographic reconstruction of the entire corona. The spatial resolution of the tomographic inversion is ultimately limited by the smearing due to coronal dynamics.

In 2003, the Solar Mass Ejection Imager (SMEI) was launched, which is designed to map largescale variations of the electron density in the heliospheric solar wind or in CMEs, by observing Thompson-scattered sunlight from a near-Earth orbit (Jackson et al., 2004). Using the additional constraint of a 3D kinematic heliospheric model, the 3D density distribution can be reconstructed with tomographic quality, a CAT method that is also called corotational tomography (Jackson and Hick, 2002, 2004).

Some serendipitous measurements with true stereoscopy using the Earth and a single interplanetary spacecraft, or with multiple interplanetary spacecraft were actually also carried out before the STEREO mission. The STEREO-1 Experiment used simultaneous radio observations from the Nançay radioheliograph and the Soviet planetary probe Mars-3 to measure the directivity pattern of solar type III bursts (Caroubalos and Steinberg, 1974). Multi-spacecraft observations from the International Sun Earth Explorer (ISEE-3), Pioneer Venus Orbiter (PVO), Helios 2, the HighEnergy Astrophysical Observatory A (HEAO-A), and Ulysses were used to measure the directivity of solar flare hard X-ray bursts (Kane, 1981; Kane et al., 1992, 1998), in conjunction with the near-Earth spacecraft Geostationary Operational Environmental Satellites (GOES) or Yohkoh.

\subsection{The STEREO mission}

Since about $90 \%$ of the literature on solar and heliospheric stereoscopy and tomography resulted from the STEREO mission at the time of writing, the STEREO mission is undoubtly the most important observatory for this scientific discipline.

The twin Solar TErrestrial RElations Observatory (STEREO) spacecraft were launched on October 26, 2006. At the end of January 2007 the two spacecraft separated and entered heliospheric orbits in opposite directions, STEREO-A(ahead) leading in east direction and STEREO-B(behind) trailing in west direction around the Sun, increasing their separation by $\approx 45^{\circ}$ per year, but maintaining their average distance of $\approx 1.0 \pm 0.1 \mathrm{AU}$ from the Sun all the time (Figure 1). At the time of writing, the two spacecraft are separated by $\approx 180^{\circ}$. These particular orbits provide solar data that are suitable for small-angle stereoscopy at the beginning of the mission (or whenever the separation angle is near zero or 180 degrees), while large-angle stereoscopy and tomography is feasible in the later years. The data rate is highest at the beginning of the mission, but drops 

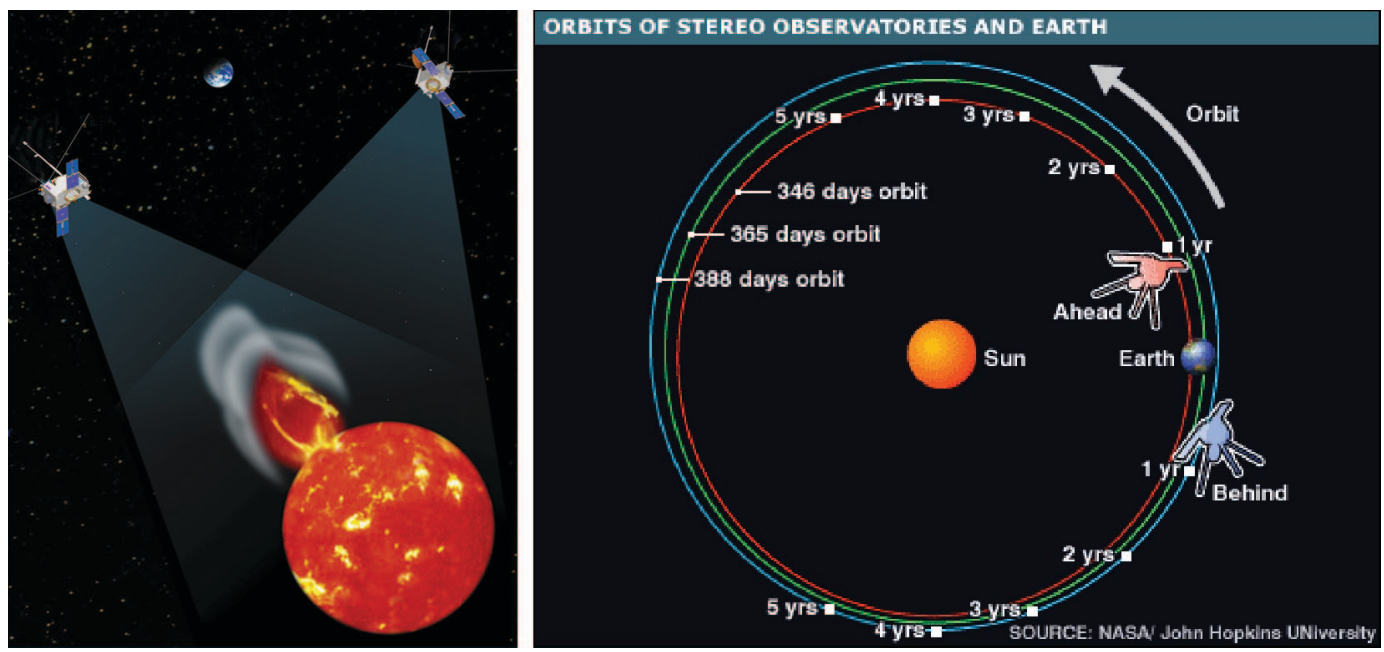

Figure 1: Left: Artists concept of the STEREO twin spacecraft 3D perspective on a coronal mass ejection launched from the Sun. Right: Orbits of STEREO-A (red) and STEREO-B (blue) with respect to the Earth's annual orbit (green) (credit: NASA).

continuously due to the lower telemetry rate later in the mission. The STEREO mission concept is described in Grigoryev (1993), Schmidt and Bothmer (1996), Socker et al. (1996), Davila et al. (1996), Davila (1998), Socker (1998), Rust (1998), Liewer et al. (1998), Bothmer et al. (1998), Socker et al. (2000), Mueller et al. (2003), Kaiser (2005), Kaiser et al. (2008), and Driesman et al. (2008).

Each of the two identical spacecraft contains a set of four instrument packages, including (i) the Sun Earth Connection Coronal and Heliospheric Investigation (SECCHI) suite (Howard et al., 2002; Michels, 2002; Howard et al., 2008), which includes the Extreme UltraViolet Imager EUVI (Wülser et al., 2004; Delouille et al., 2008), the coronagraph COR-I for 1.4-4.0 solar radii (Thompson et al., 2003; Thompson and Reginald, 2008; Thompson et al., 2010), the coronagraph COR-II for 2-15 solar radii, and the Heliospheric Imagers (HI-1) for 8-45 solar radii and (HI-2) for 35-200 solar radii (Harrison et al., 2009), (ii) the In situ Measurments of PArticles and CME Transients (IMPACT) experiment (Luhmann et al., 2008), (iii) the PLAsma and SupraThermal Ion Composition (PLASTIC) experiment (Galvin et al., 2008), and (iv) the STEREO/WAVES (SWAVES) antenna (Bougeret et al., 2008). Supporting software to analyze STEREO data from the solar corona includes the Flexible Image Transport System (FITS) world coordinate system (Thompson, 2006; Thompson and Wei, 2010) and the atomic database CHIANTI for the response function of EUVI (Young and Landi, 2009).

While the STEREO mission is already 4 years underway at the time of writing, new solardedicated imager missions came along, such as the US-Japanese mission Hinode and the NASA mission Solar Dynamics Observatory (SDO), which provide valuable context information to STEREO with unprecedented high-resolution imagery in EUV, soft X-rays, and white-light wavelengths. Especially the combination of STEREO/EUVI-A, EUVI-B, and the Atmospheric Imaging Assembly $A I A / S D O$ configure a powerful triple-viewpoint system for improved stereoscopy and tomography. 


\section{Stereoscopy and Tomography Methods}

\subsection{Solar-rotation stereoscopy}

First we are describing here stereoscopic and tomographic 3D reconstruction techniques that use only one single viewpoint (at Earth), while the aspect angle change is produced by the solar rotation.

\subsubsection{Static solar-rotation stereosocopy}

For quasi-stationary structures in the solar corona, the solar rotation with a synodic period of $T_{\text {sys }}=26.24$ days provides a natural change in aspect angle that can be exploited for stereoscopic measurements. If an image of the Sun is aligned with the solar rotation axis in y-direction and the heliographic latitude of the Sun center is small $\left(b_{0} \approx 0\right)$, the solar rotation rate introduces a displacement $\Delta x$ of a source at heliographic longitude $l_{1}$ and latitude $b_{1}$ in east-west direction as

$$
\Delta x_{12}=x\left(t_{2}\right)-x\left(t_{1}\right)=\left(R_{\odot}+h\right) \cos \left(b_{1}\right)\left[\sin \left(l_{1}+\omega_{\text {syn }}\left(t_{2}-t_{1}\right)\right)-\sin \left(l_{1}\right)\right],
$$

where $l_{1}$ is measured relative to the longitude of the observer and $\omega=2 \pi / T$ is the differential rotation rate that slightly depends on the latitude $b=b_{1}$,

$$
\omega_{\text {sid }}(b)=A+B \sin ^{2}(b)+C \sin ^{4}(b),
$$

with the coefficients $A=14.71 \pm 0.05 \mathrm{deg} / \mathrm{day}, B=-2.4 \pm 0.2 \mathrm{deg} / \mathrm{day}$, and $C=-1.8 \pm 0.3 \mathrm{deg} / \mathrm{day}$, yielding an equatorial (sidereal) rotation period of $T_{\text {sid }}=24.47$ days, which has to be multiplied with a factor of $T_{\text {syn }} / T_{\text {sid }}=26.24 / 24.47=1.0723$ for the synodic coordinate system. The height dependence of the displacement $\Delta x(t)$ in Eq. (1) yields a radial altitude measurement $h$ above the solar surface with a radius of $R_{\odot}=696,000 \mathrm{~km}$, since all other parameters $\left(x_{1}, x_{2}, l_{1}, b_{1}\right)$ can be measured at two different times $t_{1}$ and $t_{2}$, which gives a unique value for the altitude $h$, supposed that the altitude of the source does not change during the observing interval. The lower time limit of a stereoscopic measurement is given by the desired accuracy of altitude measurement $\Delta h$ and the spatial resolution $\Delta x$ of the instrument, because the stereoscopic parallax effect has to exceed the spatial resolution of the instrument, which is given by

$$
t \approx \frac{T_{\mathrm{syn}}}{2 \pi} \frac{\Delta x}{\Delta h} .
$$

Say, for a spatial resolution of $\Delta x=1^{\prime \prime}$ and an accuracy of $\Delta h=3000 \mathrm{~km} \approx 4^{\prime \prime}$ we need $t \approx 1.0$ day, which obviously exceeds most evolutionary time scales of coronal structures (typically from minutes to hours).

The first solar-rotation based stereoscopic height measurements are described in Berton and Sakurai (1985), who measured the 3D coordinates of a set of coronal loops identified in Skylab XUV images from 1973, measuring the stereoscopic parallax effect during $1-2$ days. They estimated the measuring error to $\approx 0.004 R_{\odot} \approx 3000 \mathrm{~km}$ and reported the $3 \mathrm{D}$ coordinates of a large inter-active region loop with a height of $\approx 0.15 R_{\odot}=100,000 \mathrm{~km}$, for which the loop plane inclination angle of $\theta \approx 25^{\circ}$ could be determined.

The same method of solar-rotation based stereoscopic parallax measurement has been applied to radio maps of active regions at a wavelength of $\lambda=20 \mathrm{~cm}$ (Figure 2), observed with the Very Large Array (VLA) over the course of several days (Aschwanden et al., 1992; Aschwanden and Bastian, 1994a,b; Aschwanden, 1995). While the VLA has a spatial resolution of $\approx 5^{\prime \prime}$ at this frequency $\nu \approx 1.5 \mathrm{GHz}$, the accuracy of the source positions could be optimized to $\approx 0.1^{\prime \prime}$ by cross-correlating partial source maps, which yields sub-resolution accuracy for the locations of the source centroids. Radio emission from active regions at $\nu \approx 1.5 \mathrm{GHz}$ is dominated by free-free 


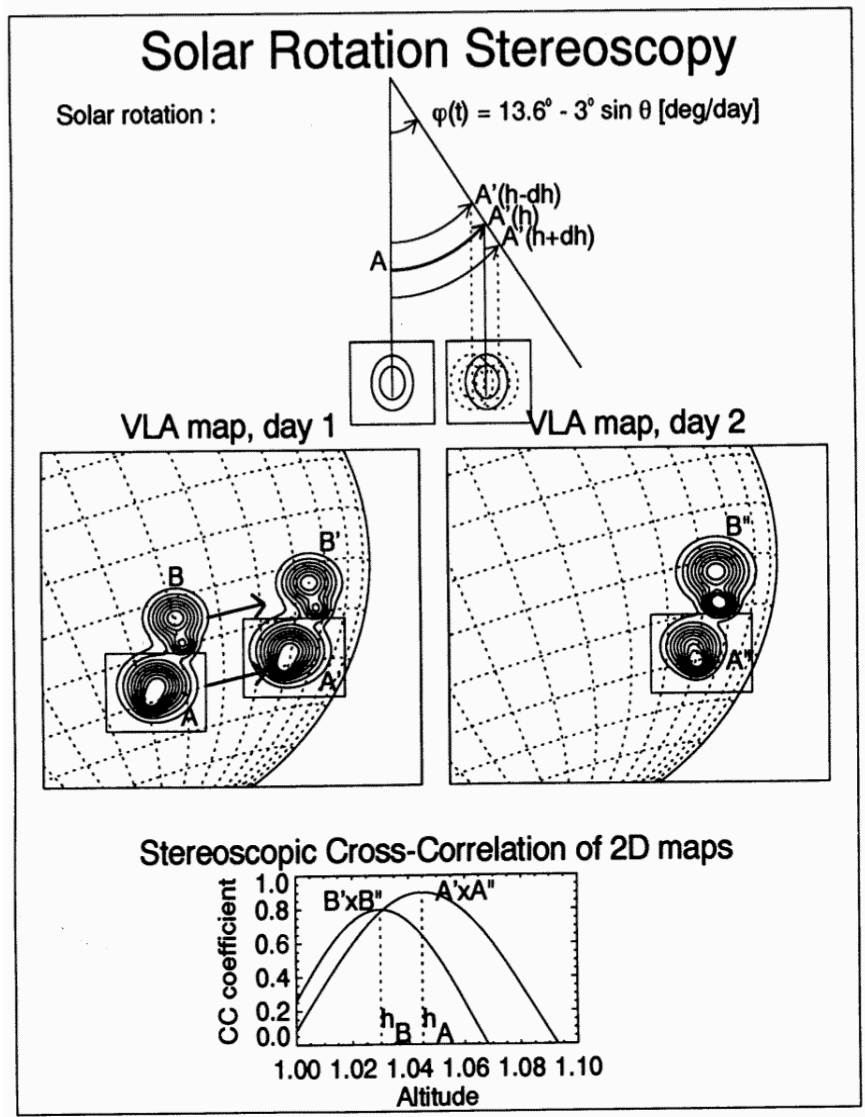

Figure 2: The principle of solar-rotation stereoscopy is illustrated with this example of radio brightness maps observed with the Very Large Array (VLA) at two different days (middle panels), where partial maps covering sources A or B (from day 1) are rotated to the locations A' and B' expected at day 2 as a function of the distance $R_{\odot}+h$ from Sun center (top), which are then cross-correlated with the actually observed maps A" and B" at day 2 (bottom), which yields a direct altitude measurement $h_{A}$ and $h_{B}$ of the source centroids (from Aschwanden and Bastian, 1994b). 
emission and, hence, the 3D centroid position corresponds to the altitude where the source becomes optically thick.

Sunspots are also relatively stable over a time interval of a day and, thus, the iso-Gauss surfaces in the lower corona. Radio mapping of sunspots at microwave frequencies of $\nu \approx 10-15 \mathrm{GHz}$ is dominated by gyro-resonance emission, which originates from dome-like iso-Gauss surfaces above sunspots, typically at harmonics of $s=2,3,4$ of the fundamental gyrofrequency of the magnetic field. Measuring the stereoscopic parallax of the dominant gyroresonance source above a sunspot over the course of 4 days with the Owens Valley Radio Observatory (OVRO), the altitudes of the gyroresonant layers could be determine for a set of 7 frequencies in the range of $\nu \approx 10-15 \mathrm{GHz}$, which were found in a height range of $h \approx 3000-12,000 \mathrm{~km}$, and allowed to constrain the local magnetic field with a potential field model and to identify the correct harmonics at every frequency (Aschwanden et al., 1995). Of course, stationarity of the magnetic field over time scales of 4 days can only be expected for stable large sunspots, while small-scale magnetic fields change during much shorter time scales in active regions.

\subsubsection{Dynamic solar-rotation stereosocopy}

Coronal loops are generally not stable over a time period of a day, because the heating rate appears to be discontinuous and intermittent and cooling by conductive and radiative loss occurs on time scales of less than an hour. This means that loops at the same location observed a day apart are not identical, but continuously replaced by plasma upflows and downflows. However, what stays more permanent is the magnetic field, say over time scales of a few days, especially the large-scale dipolar fields that represent the main magnetic field structure of an active region, produced by the leading sunspot and the trailing region with opposite magnetic polarity. The property of the quasistationarity of the magnetic field can thus be used to constrain the 3D geometry of near-cospatial loops at different times, regardless how often the plasma is flushed through the magnetic conduits. A technique that uses the stable 3D geometry of the magnetic field and is not sensitive to the fast-paced plasma dynamics has been developed for the 3D reconstruction of a set of dipolar loops that make up an active region, called dynamic stereoscopy (Figure 3), which was applied to a few days of SOHO/EUV images (Aschwanden et al., 1999, 2000). This method allows us to determine the approximate 3D geometry of coronal loops under the assumption of planarity, which requires only one free parameter (the inclination angle of the loop plane) to determine the 3D coordinates of loops from the observed 2D projections $[x(s), y(s)]$ as a function of the loop length coordinate $s$.

\subsection{Solar-rotation tomography}

There are three wavelength regimes where solar-rotation tomography has been applied, but each one requires a different treatment of the radiation: tomography of (i) optically thin emission in EUV and soft X-rays, (ii) Thompson-scattered white light, or (iii) radio wavelengths. These tomographic 3D reconstruction techniques are using only one single viewpoint (at Earth), while the aspect angle change is produced by the solar rotation.

\subsubsection{EUV and Soft X-rays}

Let us consider first the simplest method of optically thin EUV or soft X-ray emission. The brightness of the solar corona observed in EUV or soft X-rays is produced by optically thin freefree emission, which can be characterized by an emission measure $E M$ that is proportional to the squared electron density $n_{e}$ and column depth along the line-of-sight at a given temperature $T$ (assuming that the plasma is in local thermal dynamic equilibrium, which is not always the case 


\section{Principle of Dynamic Stereoscopy}
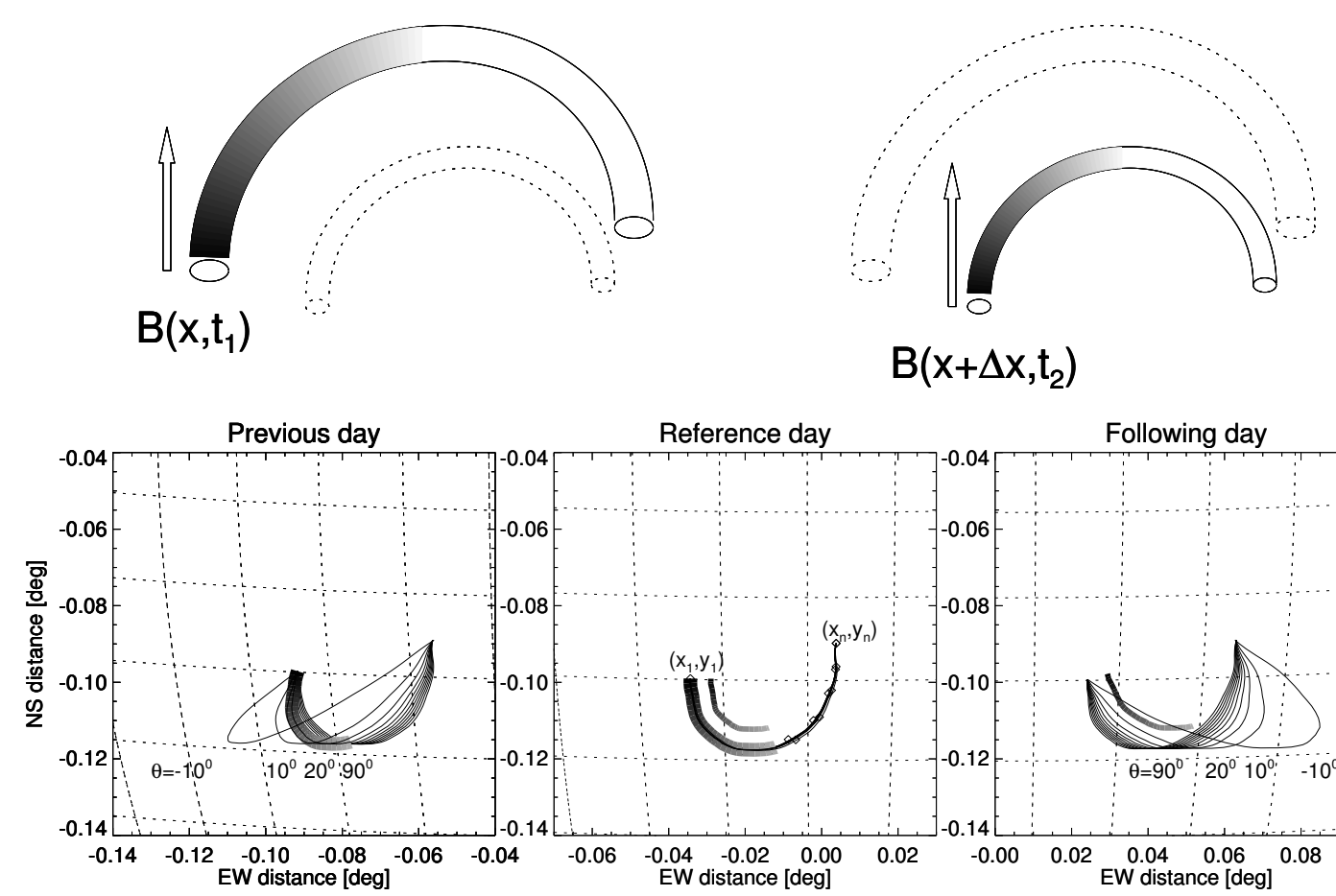

$\mathrm{B}\left(\mathrm{x}+\Delta \mathrm{x}, \mathrm{t}_{2}\right)$

Stripe aligned with projection $\theta=10^{\circ}$
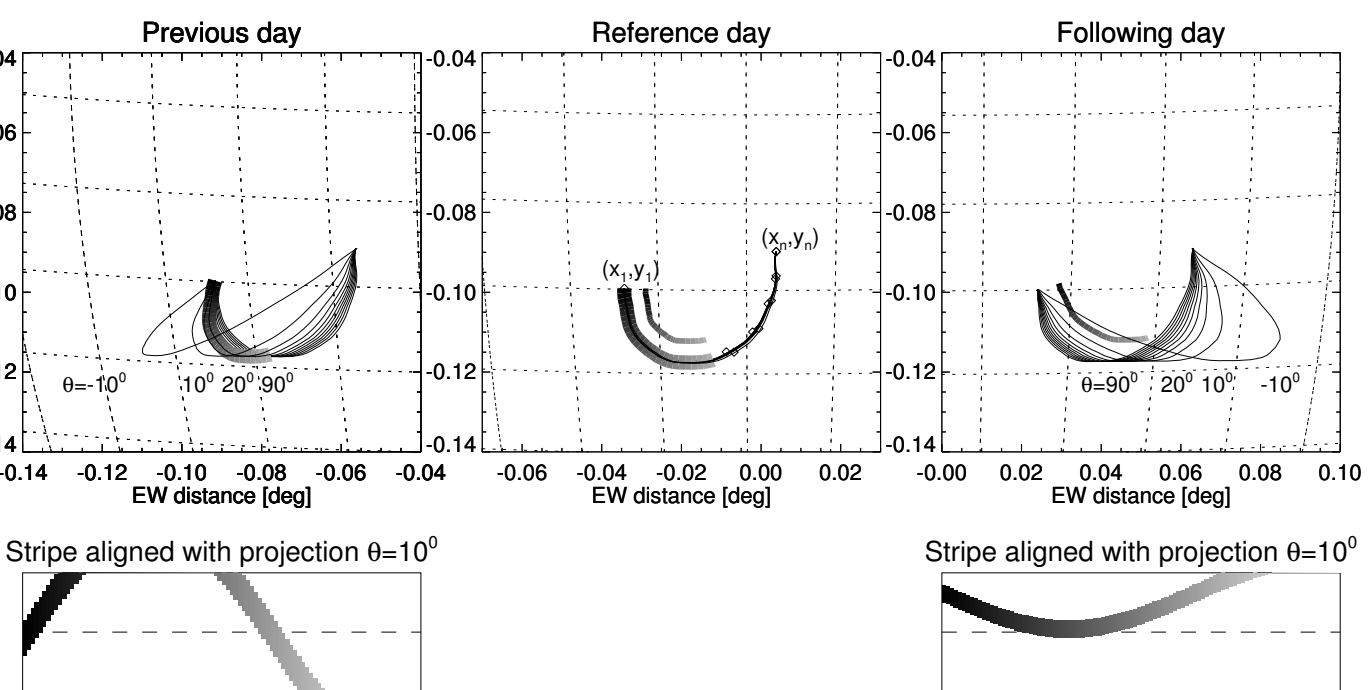

Stripe aligned with projection $\theta=10^{0}$

Stripe aligned with projection $\theta=20^{\circ}$ Stripe aligned with observed projection Stripe aligned with projection $\theta=20^{\circ}$
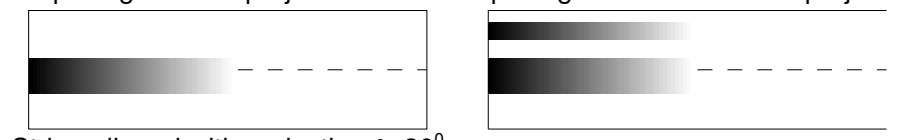

Stripe aligned with projection $\theta=30^{\circ}$
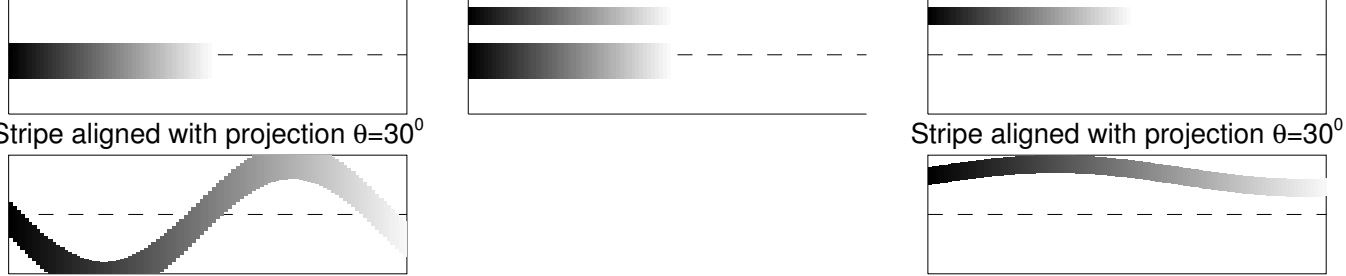

Stripe aligned with projection $\theta=30^{\circ}$

Figure 3: The principle of dynamic solar-rotation stereoscopy is illustrated for an example of two adjacent loops (top). The only free parameter in the 3D reconstruction is the loop inclination plane, which yields different $2 \mathrm{D}$ projections at 3 consecutive days (middle). The best-fitting inclination angle can be determined by optimizing the (parallel) co-alignment between the observed and model loop stripe as a function of the inclination angle (bottom) (from Aschwanden et al., 1999). 
for loops undergoing rapid heating),

$$
E M \propto \int n_{e}^{2}(z, T) d z
$$

while the intensity or flux $F_{\lambda}(x, y)$ measured at a wavelength $\lambda$ represents the temperature integral of the differential emission measure $d E M / d T \propto n_{e}^{2} d z / d T$ multiplied with the filter response function $R_{\lambda}(T)$,

$$
F_{\lambda}=\int \frac{d E M(T)}{d T} R_{\lambda}(T) d T,
$$

which defines the general inversion problem for the 3D density distribution $n_{e}(x, y, z)$, for a given temperature filter $\lambda$,

$$
F_{\lambda}\left(X_{i}, y_{j}, \theta_{k}\right)=\sum_{l} A_{\lambda} n_{e}^{2}\left(X_{i}, y_{j}, Z_{l}\right)+\sigma_{\lambda}\left(X_{i}, y_{j}, \theta_{k}\right)
$$

with $A_{\lambda}$ being the conversion factor of the squared density $n_{e}^{2}$ to the observed brightness $F_{\lambda}$ (as defined by Eqs. (4) and (5)), and $\sigma_{\lambda}$ is the data noise. The absolute coordinate system with origin in the Sun center is given in coordinates $\left(x_{i}, y_{j}, z_{k}\right)$ with the $x$-axis co-aligned to the solar rotation direction, while $\left(X_{i}, y_{j}\right)$ is the image coordinate system rotated around the solar axis $(y$-axis $)$ by the line-of-sight angle $\theta_{k}$ according to,

$$
\left(\begin{array}{c}
X_{i} \\
Z_{l}
\end{array}\right)=\left(\begin{array}{rr}
\cos \theta_{k} & -\sin \theta_{k} \\
\sin \theta_{k} & \cos \theta_{k}
\end{array}\right)\left(\begin{array}{c}
x_{i} \\
z_{l}
\end{array}\right)
$$

Thus, if we have an image with a size of $N^{2}$ pixels and want to reconstruct the $3 \mathrm{D}$ density distribution $n_{e}(x, y, z)$ with $N^{3}$ voxels, the problem is under-constrained. Using the variation of the aspect angle, for instance by taking multiple images $\left(N_{k}\right)$ over a time interval with significant solar rotation (of the aspect angle $\theta_{k}$ ), will increase the number of constraints to $N^{2} N_{k}$, as long as the reconstructed volume is static, but a unique solution would theoretically require $N_{k}=N$ images, which is not feasible in practice. The inversion of the $3 \mathrm{D}$ density distribution is therefore always under-constrained and requires special inversion techniques with additional constraints. One way to reduce the degrees of freedom is to reduce the solution volume by using a spherical coordinate system aligned with the solar surface and by restricting the number or radial voxels to a small number that covers only about the lowest density scale height, see for instance Frazin et al. (2009b) (Figure 15, bottom left).

The simplest tomographic reconstruction method is the backprojection method, which yields a probability distribution based on the linear addition of projections from different directions (Figure 4). Another (under-constrained) inversion method that has been used for solar tomography is the robust, regularized, positive estimation scheme (Frazin, 2000; Frazin and Janzen, 2002). The combination of differential emission measure analysis (Eq. (4)) and solar rotation tomography allows in principle to reconstruct the average density $n_{e}(x, y, z)$ and temperature $T_{e}(x, y, z)$ in each voxel (Frazin et al., 2005b; Frazin and Kamalabadi, 2005b). Besides the problem of underconstrained inversion, the time variability is an additional challenge, which could be overcome with Kalman filtering (Frazin et al., 2005a). The first attempt to reconstruct the 3D density distribution in the solar corona by means of solar-rotation tomography was done using a 2-week's dataset of soft X-ray images from Yohkoh (Hurlburt et al., 1994).

\subsubsection{White-light}

Let us consider the second wavelength regime, i.e., white light, which undergoes Thompson scattering in the solar corona, which is sensitive to the geometry of the distribution of scattering particles 


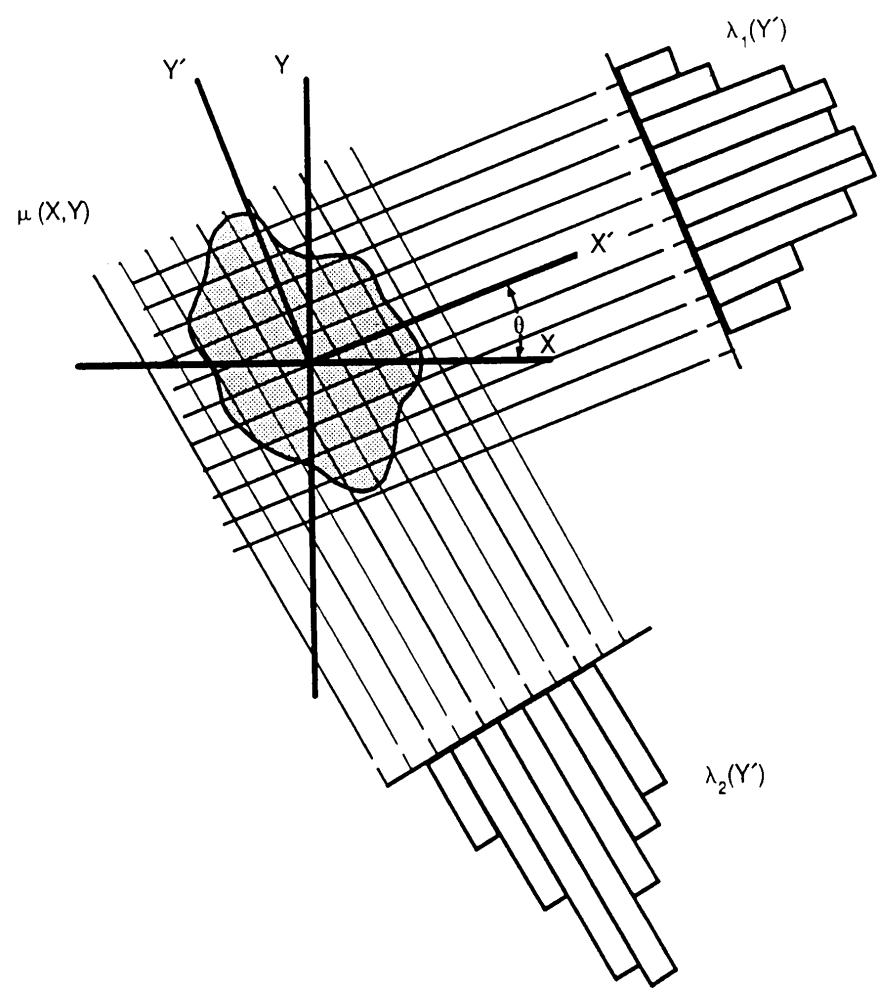

Figure 4: The principle of the backprojection method used in medical tomography is visualized for the case of two 1-D projections observed from two arbitrary directions (angle $\theta$ ), from which the unknown true 2-D brightness distribution is reconstructed (from Davila and Thompson, 1992).

and the direction to the observer. The scattering cross-section depends on the angle $\chi$ between the line-of-sight and the radial direction through the scattering electron as (Jackson, 1962),

$$
\frac{d \sigma}{d \omega}=\frac{1}{2} r_{e}^{2}\left(1+\cos ^{2} \chi\right)
$$

where $d \sigma / d \omega$ is the differential cross-section in units of $\left[\mathrm{cm}^{2} \mathrm{sr}^{-1}\right]$ and $r_{e}=e^{2} / m_{e} c^{2}=2.82 \times$ $10^{-13} \mathrm{~cm}$ is the classical electron radius. By integrating over all solid angles we obtain the total cross-section for perpendicular scattering, the so-called Thompson cross-section for electrons,

$$
\sigma_{T}=\frac{8 \pi}{3} r_{e}^{2}=6.65 \times 10^{-25} \mathrm{~cm}^{2} .
$$

The total scattered radiation $I(x, y)$ can then be calculated by integrating over the source locations of the photons (the photosphere) and the scattering electrons (with a $3 \mathrm{D}$ distribution $n_{e}(x, y, z)$ ) along the line-of-sight $z$, as a function of the scattering angle $\chi(x, y, z)$ with respect to the observers line-of-sight, which was first calculated by Minnaert (1930), van de Hulst (1950), and Billings (1966). For a recent review see Howard and Tappin (2009a). The degree of polarization $p$, which is observed in the polarized brightness $(p B)$ component of white-light images, is defined as,

$$
p=\frac{I_{T}-I_{R}}{I_{T}+I_{R}}=\frac{I_{P}}{I_{\mathrm{tot}}},
$$

where $I_{T}$ and $I_{R}$ represent the tangential and radial terms of the total scattered radiation, as given in Minnaert (1930), van de Hulst (1950), Billings (1966), or Howard and Tappin (2009a). Many 
coronagraphs (such as those on STEREO) have the capability to measure linear polarization in three orientations, from which the total $I_{\text {tot }}$ and polarized brightness $I_{P}$ can be derived. The total scattered radiation is proportional to the electron density $n_{e}(x, y, z)$ of the scattering corona, in contrast to the square-dependence of the observed brightness on the density (Eq. (4)) for free-free emission in soft X-rays and EUV.

Since the differential cross section $d \sigma / d \omega$ (Eq. (8)) varies only by a factor of two with angle $\chi$, the plane-of-the-sky or plane-of-max-scattering approximations are very poor. It is essential for any tomography approach which aims to reconstruct an extended density distribution to treat the observations as the result of an extended integral along the line-of-sight. The plane of maximum scattering has often been approximated with the plane-of-sky in the past, which is appropriate for locations near the solar limb, but needs to be corrected with the actual plane of maximum scattering, for sources at large distances from the Sun, indicated with the impact radius $d$ in Figure 5 (Vourlidas and Howard, 2006). This correction becomes relevant for tomographic 3D reconstruction of CMEs that propagate far away from the Sun. Using a time series of images in polarized brightness $I_{P}$ taken over a time interval with significant solar rotation (which varies the line-of-sight angles) allows then to deduce the 3D density distribution $n_{e}(x, y, z)$ in a coronal volume.

The first tomographic reconstruction of the 3D density distribution of the solar corona, based on a time series of coronagraph images from Skylab in white light has been accomplished by Altschuler (1979). Tomographic inversion of coronagraph images from Mark III K-coronameter on Hawaii and from LASCO C-1 were conducted by Zidowitz et al. (1996), Zidowitz (1997), and Zidowitz (1999). Tomography of the solar corona in an altitude range of a few solar radii has been systematically investigated by regularization inversion methods (e.g., Frazin, 2000; Frazin and Kamalabadi, 2005b), and applied to LASCO C-2 datasets (Frazin and Janzen, 2002; Morgan et al., 2009; Morgan and Habbal, 2010), to Mauna Loa Solar Observatory Mark-IV coronameter data (Butala et al., 2005), to STEREO COR-1 datasets (Kramar et al., 2009; Barbey et al., 2011), and by separating contributions of the K- and F-corona (Frazin and Kamalabadi, 2005a).

\subsubsection{Radio wavelengths}

Solar radio emission has many different emission mechanisms, which moreover have complicated properties depending on the frequency (or wavelength), polarization, opacity, and magnetic field (for an overview see, e.g., Chapter 15 in Aschwanden, 2005, and references therein). For 3D tomography it matters a lot whether radio emission is observed in an optically thin or thick regime. If an optically thin feature is observed (e.g., free-free emission at decimetric frequencies), the radio brightness has to be calculated from a line-of-sight integral with varying opacity, similar as for EUV or soft X-ray wavelengths. In contrast, if optically thick emission is observed (e.g., gyroresonance emission in microwaves), the radio brightness originates from a localized source surface that can be treated like an opaque body in the $3 \mathrm{D}$ reconstruction.

Let us consider the case of free-free (bremsstrahlung) emission, which has a free-free absorption coefficient $\alpha_{\nu}(z)$ for thermal electrons that depends on the ambient ion density $n_{i}(z)$, electron density $n_{e}(z)$, and temperature $T_{e}(z)$, at position $z$ along a given observer's line-of-sight, and radio frequency $\nu$ as,

$$
\alpha_{f f}(z, \nu) \approx 9.786 \times 10^{-3} \frac{n_{e}(z) \sum_{i} Z_{i}^{2} n_{i}(z)}{\nu^{2} T^{3 / 2}(z)} \ln \Lambda,
$$

where $\ln \Lambda(z) \approx 20$ is the Coulomb integral, which yields the free-free opacity $\tau_{f f}(z, \nu)$ as a function of position $z$ by integrating over the column depth range $z^{\prime}=[-\infty, z]$,

$$
\tau_{f f}(z, \nu)=\int_{-\infty}^{z} \alpha_{f f}\left[T_{e}\left(z^{\prime}\right), n_{e}\left(z^{\prime}\right), \nu\right] d z^{\prime} .
$$




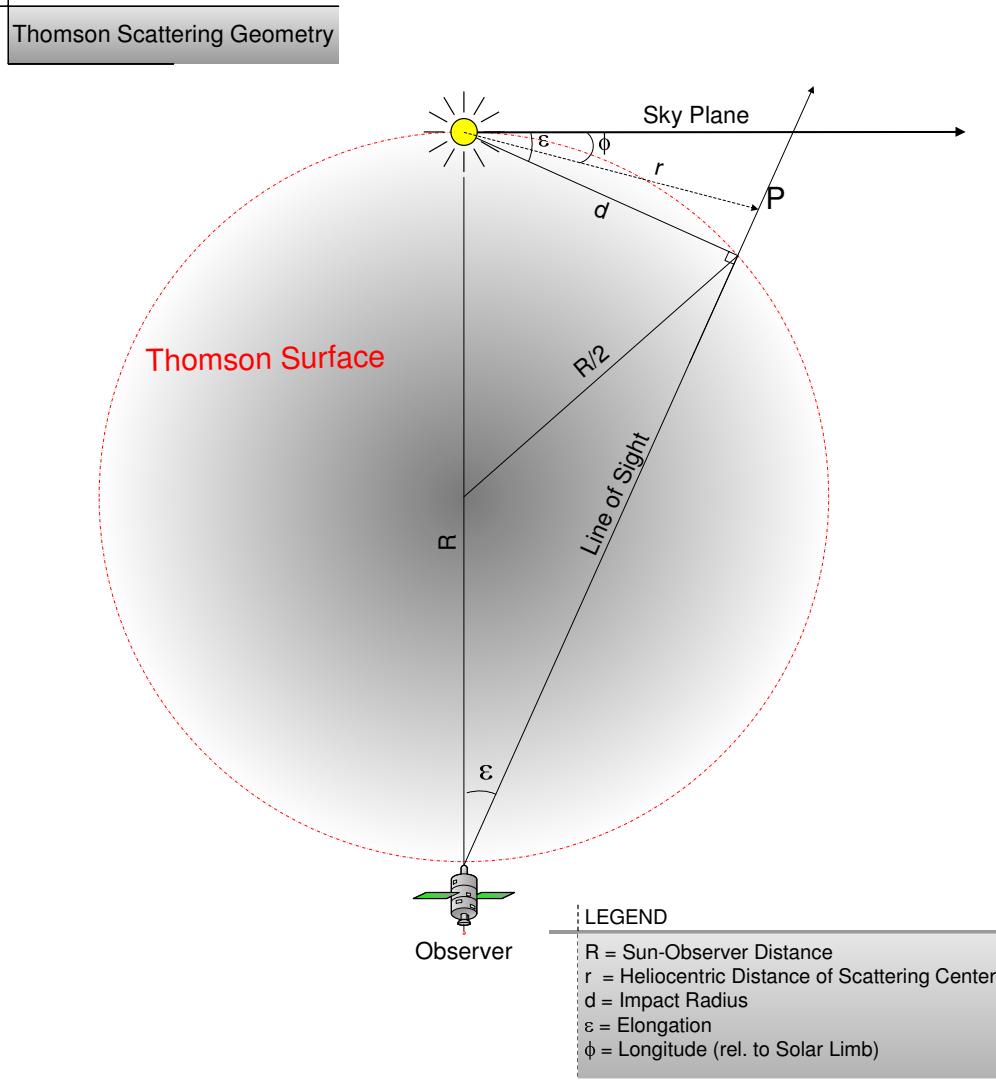

Figure 5: Generalized Thompson scattering geometry: The spherical Thompson surface represents the source locations of the maximum $\left(90^{\circ}\right)$ scattering angle with respect to an observer at distance $R$. The white-light brightness has to be integrated over all scattering positions $P$ along a given line-of-sight (from Vourlidas and Howard, 2006). 
From the free-free opacity we obtain the radio brightness temperature $T_{B}(\nu)$ at the observer's frequency $\nu$ with a further integration of the opacity along the line-of-sight,

$$
T_{B}(\nu)=\int_{-\infty}^{0} T_{e}(z) \exp ^{-\tau_{f f}(z, \nu)} \alpha_{f f}(z, \nu) d z .
$$

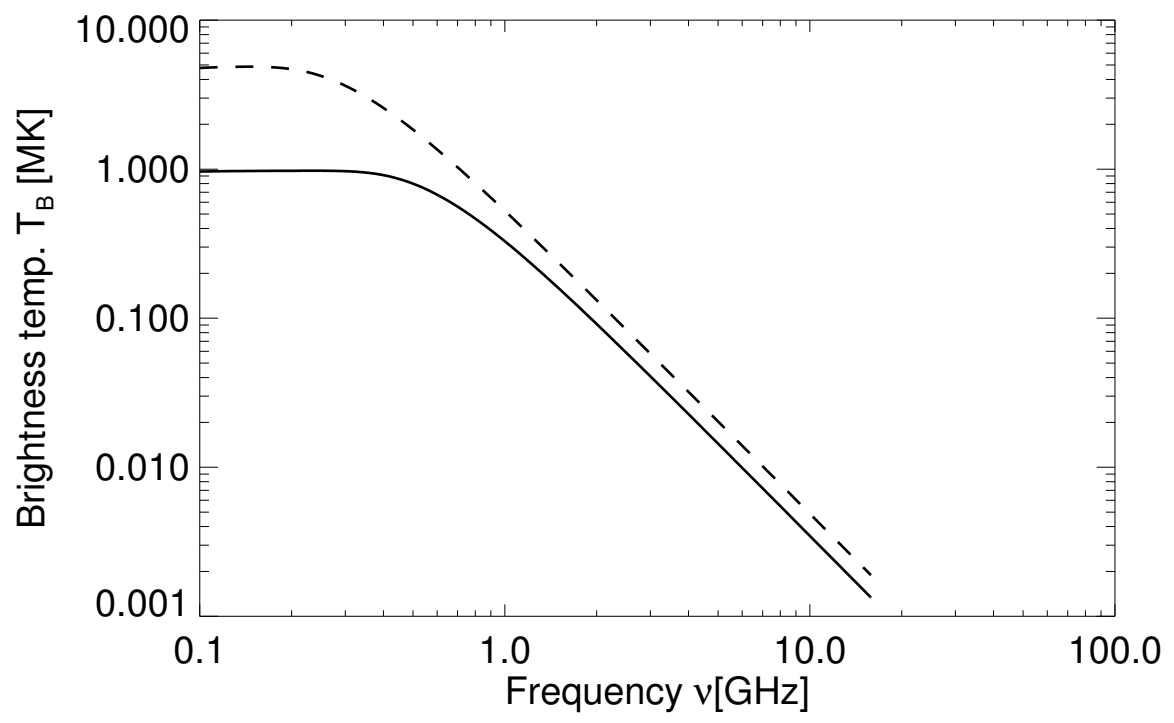

Figure 6: Quiet-Sun brightness temperature spectrum for an isothermal corona with $T_{e}=1.0 \mathrm{MK}$ (solid line) or $T_{e}=5.0 \mathrm{MK}$ (dashed line) with a base density of $n_{0}=10^{9} \mathrm{~cm}^{-3}$ and gravitational stratification (with a density scale height of $\lambda \approx 50\left(T_{e} / 1 \mathrm{MK}\right) \mathrm{Mm}$ ) (from Aschwanden et al., 2004).

An example of a quiet-Sun radio brightness spectrum $T_{B}(\nu)$ is given in Figure 6 for different coronal temperatures in hydrostatic equilibrium. The observed quantity is the flux density $I(\nu)$, which can be calculated from the brightness temperature $T_{B}(\nu)$ with the Rayleigh-Jeans approximation at radio wavelengths, integrated over the solid angle $\Omega_{S}$ of the radio source,

$$
I(\nu)=\frac{2 \nu^{2} k_{B}}{c^{2}} \int T_{B} d \Omega_{S} .
$$

These expressions describe how the radio brightness observed at a particular frequency $\nu$ depends on the $3 \mathrm{D}$ density $n_{e}(x, y, z)$ and temperature distribution $T_{e}(x, y, z)$ of an observed source (e.g., an active region), and this way describes the inversion problem that has to be solved to obtain the $3 \mathrm{D}$ density distribution $n_{e}(x, y, z)$. One way to obtain a large number of observational constraints, is to synthesize radio images at many frequencies $\nu$, a method that is called frequency tomography (Aschwanden et al., 1992; Aschwanden, 1995), which was pioneered with the multi-frequency imaging radio interferometer RATAN-600 with up to 36 frequencies in the $\nu=0.1-5.0 \mathrm{GHz}$ range (Bogod and Grebinskij, 1997; Gelfreikh, 1998; Grebinskij et al., 2000), and has been simulated for a proposed Frequency-Agile Solar Radiotelescope (FASR) (Aschwanden et al., 2004). However, the frequency tomography method allows only to invert the electron density $n_{e}(\nu)$ and temperature $T_{e}(\nu)$ as a function of the radio frequency $\nu$, while an additional opacity model $\nu(z)$ is required to map the radio frequency into a geometric coordinate $z$ for each line-of-sight. Such additional information on the absolute height $h(\nu)=z(\nu) \cos \theta$ (at an angle $\theta$ from Sun center) can be obtained from solar-rotation stereoscopy (see Section 3.1). 

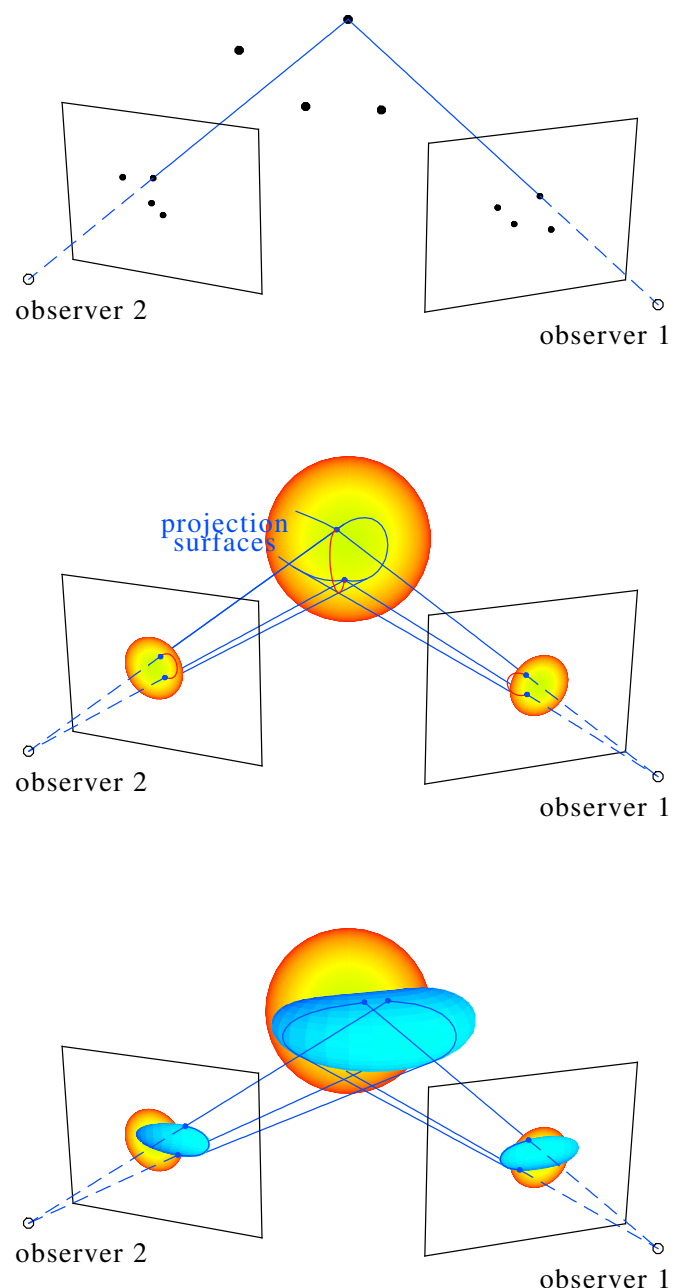

Figure 7: Triangulation of point-like 1D (top), curvi-linear 2D (middle), and voluminous 3D (bottom) structures. The triangulation and back-projection of point-like and curvi-linear structures is unique for two stereoscopic viewpoints, while two projections of a voluminous structure do not unambiguously define the 3D surface or volume (from Inhester, 2006). 


\subsection{Stereoscopic triangulation or tie-point method}

We are turning now to "true stereoscopy", where an object is simultaneously observed from multiple aspect angles, rather than using the solar rotation to vary the aspect angle over time. Further, we distinguish between stereoscopic triangulation, which can only be applied to point-like or curvilinear structures (Figure 7), and stereoscopic tomography, where a full 3D density distribution of a voluminous structure is obtained from simultaneous multiple aspect angles. An introductory primer for solar stereoscopy is given in Inhester (2006).

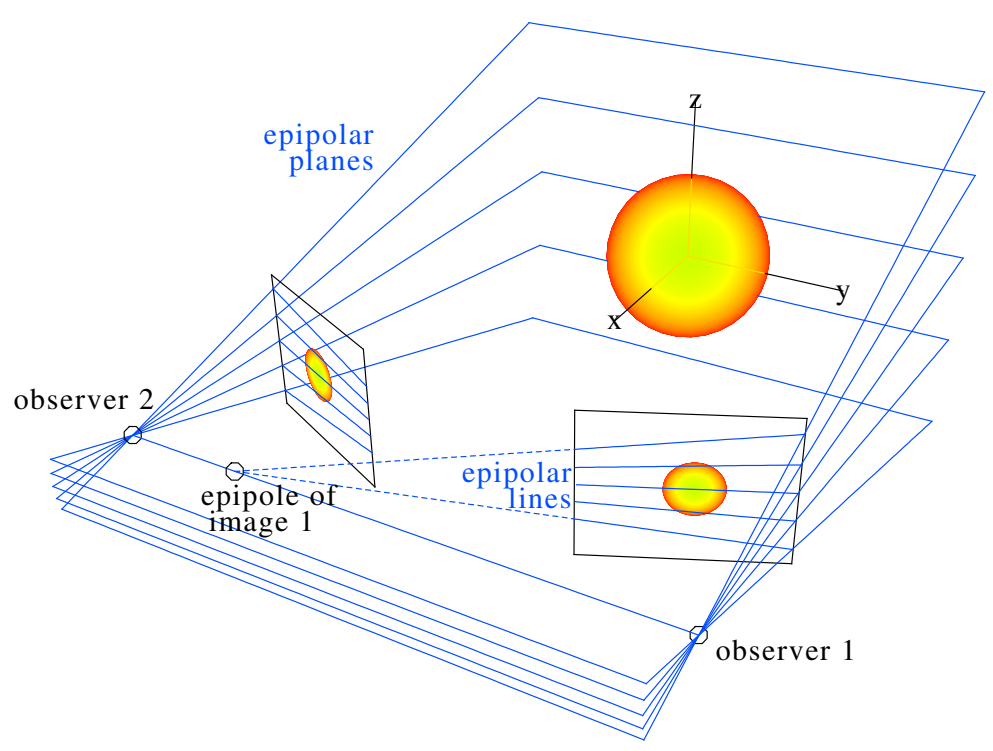

Figure 8: Orientation of epipolar planes in space and the respective epipolar lines in the images for two observers (e.g., STEREO spacecraft A and B) looking at the Sun (from Inhester, 2006).

Regarding solar stereoscopy, there exist indeed point-like objects in the solar corona (such as bright points, flare kernels, or centroids of near-spherical CME bubbles) as well as curvi-linear structures (coronal loops, fans, jets, filaments, prominences, plumes), which are suitable for stereoscopic triangulation. The process of stereoscopic triangulation generally requires three steps: (1) coalignment of a stereoscopic image pair into an epipolar coordinate system $[X, Y]$ (Figure 8); $(2)$ identification of corresponding point-like or curvi-linear features in each image (with coordinates $\left(X_{A}, Y_{A}\right)$ and $\left(X_{B}, Y_{B}\right)$; and (3) geometric triangulation to retrieve the 3-coordinates $(x, y, z)$ of the structure. The first step involves rectification of the stereoscopic pair of images to coalign the $\mathrm{X}$-axis of each one with the epipolar plane (defined by the surface that intersects the two stereoscopic observers and the Sun center), plus rescaling of the pixel size in case the two observers have different distances from the Sun. The second step involves a parameterization of the coordinates of a curvi-linear feature, say as a function of the length coordinate $s$, yielding two sets of 2D coordinates $\left[X_{A}(s), Y_{A}(s)\right]$ and $\left[X_{B}(s), Y_{B}(s)\right]$. The identification of the correct structure B in the second image that corresponds to the feature $\mathrm{A}$ in the first image is easy for small stereoscopic angles, because the two images $\mathrm{A}$ and $\mathrm{B}$ look very similar, but becomes increasingly difficult and ambiguous with larger stereoscopic angles. In practice, approximate 3D magnetic field models can be used to identify the correct correspondence of coronal features. The third step of triangulating the $3 \mathrm{D}$ coordinates is straightforward in an epipolar coordinate system, which can be calculated separately for each epipolar plane, corresponding to a particular loop position $s$, as described in 
the next paragraph for the case of two spacecraft A and B with different distances to the Sun.

We define a coordinate system $(X, Y, Z)$ that has the origin $O$ in the Sun center, the $Z$-axis is the line-of-sight from spacecraft A to Sun center, and the $X Z$ plane coincides with the plane of the spacecraft A and B (Figure 9 left). The two spacecraft have distances of $d_{A}$ and $d_{B}$ from the Sun center and observe a point $P$ at an angle of $\alpha_{A}$ and $\alpha_{B}$ in $X$-direction from Sun center (corresponding to the difference of x-pixels in the image) and at an angle $\delta_{A}$ and $\delta_{B}$ in $Y$-direction from Sun center (corresponding to difference in y-pixels in the image). The spacecraft have a separation angle of $\alpha_{\text {sep }}$ in the $X Z$ plane. The point $P$ has the $3 \mathrm{D}$ coordinates $(x, y, z)$ or heliographic longitude $l$ and latitude difference $b$ with respect to the central meridian defined by the line-of-sight axis of spacecraft $\mathrm{A}$. The projected positions of the point $P$ on the $\mathrm{X}$-axis are $x_{A}$ from spacecraft $\mathrm{A}$ and $x_{B}$ from spacecraft $\mathrm{B}$. So, our main problem is to solve for the variables $(x, y, z)$ using the observables $\left(d_{A}, d_{B}, \alpha_{A}, \alpha_{B}, \delta_{A}, \delta_{B}, \alpha_{\text {sep }}\right)$.

In the triangles $\left(O, A, x_{A}\right)$ and $\left(O, B, x_{B}\right)$ we can determine the angles $\gamma_{A}$ and $\gamma_{B}$ simply from the geometric rule that the sum of the three angles in a planar triangle amounts to $\pi$, i.e.,

$$
\gamma_{A}=\frac{\pi}{2}-\alpha_{A}
$$

and

$$
\gamma_{B}=\frac{\pi}{2}-\alpha_{B}-\alpha_{\text {sep }}
$$

Using the sine relation in a planar triangle $[a / \sin (\alpha)=b / \sin (\beta)=c / \sin (\gamma)]$ we obtain the sides $x_{A}$ and $x_{B}$ in the two triangles,

$$
x_{A}=d_{A} \tan \left(\alpha_{A}\right),
$$

and

$$
x_{B}=d_{B} \frac{\sin \alpha_{B}}{\sin \gamma_{B}} .
$$

Furthermore we have the relations in the rectangular triangles, $\tan \gamma_{A}=z /\left(x_{A}-x\right)$ and $\tan \gamma_{B}=$ $z /\left(x_{B}-x\right)$, from which we can determine the coordinates $x$,

$$
x=\frac{x_{B} \tan \gamma_{B}-x_{A} \tan \gamma_{A}}{\tan \gamma_{B}-\tan \gamma_{A}},
$$

and $z$,

$$
z=\left(x_{A}-x\right) \tan \gamma_{A} .
$$

The coordinate $y$ can be obtained from a relation in the $Y Z$ plane (Figure 9 right),

$$
y=\left(d_{A}-z\right) \tan \delta_{A} .
$$

The distance of point $P$ from the Sun center $O$ is then

$$
r=\sqrt{x^{2}+y^{2}+z^{2}}
$$

and the height of the point at position $P$ is

$$
h=r-R_{\odot} .
$$

The method of stereoscopic triangulation is also called tie-point method, because a second point $\left[X_{B}\left(s_{i}\right), Y_{B}\left(s_{i}\right)\right]$ in image $\mathrm{B}$ is tied to a first point $\left[X_{A}\left(s_{i}\right), Y_{A}\left(s_{i}\right)\right]$ in image $\mathrm{A}$, which enables triangulation of each point according to its epipolar plane. Further details on stereoscopic triangulation can be found in the tutorial of Inhester (2006), which discusses also the identification and matching problem, the tie-point reconstruction, reconstruction errors, ambiguities in identifying corresponding structures, and examples applied to SOHO/EIT data. 

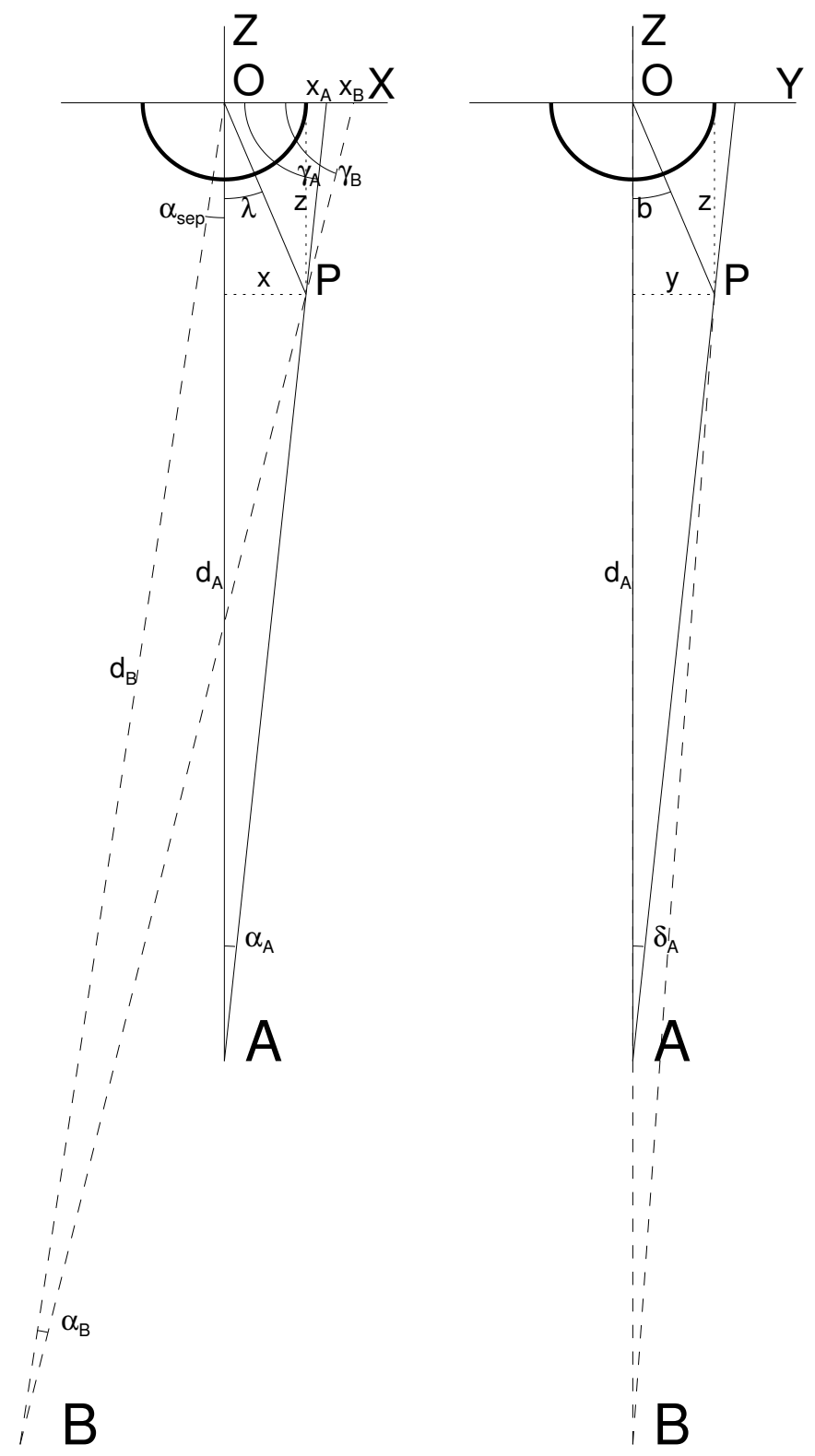

Figure 9: The geometry of triangulating or projecting a point $\mathrm{P}$ from spacecraft $\mathrm{A}$ and $\mathrm{B}$ is shown, where the (epipolar) XZ plane is coincident with the Sun center position O and the two spacecraft positions $\mathrm{A}$ and $\mathrm{B}$ (left panel), while the vertical YZ plane is perpendicular (right panel). The distances of the spacecraft from the Sun are $d_{A}$ and $d_{B}$, the observed angles of point $\mathrm{P}$ with respect to the Sun center $\mathrm{O}$ are $\alpha_{A}$ and $\alpha_{B}$, intersecting the $\mathrm{X}$-axis at positions $x_{A}$ and $x_{B}$ with the angles $\gamma_{A}$ and $\gamma_{B}$. The spacecraft separation angle is $\alpha_{\text {sep }}$. The point $\mathrm{P}$ has the $3 \mathrm{D}$ coordinates $(x, y, z)$ and heliographic longitude $l$ and latitude $b$ (from Aschwanden et al., 2008b). 
First stereoscopic triangulations of coronal loops using STEREO/A and B have been performed for 30 coronal loops in an active region observed on 2007 May 9 with a spacecraft separation angle of $\alpha_{\text {sep }}=7.3^{\circ}$ (Aschwanden et al., 2008c), and for 9 loops observed on 2007 Jun 8 with a spacecraft separation angle of $\alpha_{\text {sep }}=12^{\circ}$ (Feng et al., 2007a). Further stereoscopic triangulations have been applied to oscillating loops (Aschwanden, 2009c), to polar plumes (Feng et al., 2009), to an erupting filament (Liewer et al., 2009), or to an erupting prominence (Bemporad, 2009), including a strongly rotating, erupting, quiescent polar crown prominence (Thompson, 2011).

\subsection{Magnetic stereoscopy}

Stereoscopic triangulation of curvi-linear coronal structures (such as loops, fans, jets, filaments, plumes) can usually only be carried out for segments and a limited number of structures within relatively isolated zones that are not too much congested (subject to confusion and mis-identification of stereoscopic correspondence). One additional a priori information that can be used to enhance stereoscopic 3D reconstruction is the auxiliary use of magnetic field models. Of course, the fundamental limitation is that we still do not have perfect magnetic field models for the solar corona, but a lot of progress has been made to improve magnetic field models based on stereoscopic information, and to beat down the discrepancy between theoretical magnetic field models and observed stereoscopically triangulated loop 3D coordinates.

An early experiment with combined stereoscopy and magnetic field modeling has been conducted with multi-frequency radio maps observed with the Owens Valley Radio Observatory (Aschwanden et al., 1995). For a given 3D magnetic field, the gyroresonance layers at each frequency $\nu$ and harmonic $s=2,3,4$ form a curved 2D surface that are layered like onion shells in the lower corona above sunspots, as shown for three frequencies $(\nu=5,8,11 \mathrm{GHz})$ in Figure 10 . Some features (e.g., the brightest locations or centroids) of these gyroresonance layers can be either stereoscopically triangulated (using the solar rotation) or be modeled by matching the contours of their brightness distribution for a given projection. Such a method successfully demonstrated that in a strongly sheared region only a nonlinear force-free field could explain the observed radio brightness distribution, while a potential-field model failed (Lee et al., 1999). Also the polarization of gyroresonance emission in the theory of wave mode coupling in quasi-transverse regions was tested with combined radio mapping and magnetic field modeling (Ryabov et al., 2005).

The method of using the observed projections of coronal EUV loops to constrain a magnetic field model was proposed by Wiegelmann and Neukirch (2002) in the pre-STEREO area, who varied the $\alpha$-parameter in a force-free magnetic field extrapolation to optimize the match to observed loop locations. Magnetic modeling was then also used to constrain tomographic reconstruction of the coronal density distribution (Wiegelmann and Inhester, 2003; Ruan et al., 2008). A variety of coronal magnetic field models, such as potential, linear, and nonlinear force-free field (NLFFF) models, were used to resolve ambiguities in stereoscopic triangulation of loops (Wiegelmann and Inhester, 2006; Feng et al., 2007b; Conlon and Gallagher, 2010), reviewed in Wiegelmann et al. (2009). Comparisons of potential-field and NLFFF models with STEREO and Hinode images were also calculated for the global corona (Petrie et al., 2011; Schrijver and Title, 2011).

The success of magnetic stereoscopy depends on the quality of theoretical magnetic field models. A critical assessment of NLFFF models identified a substantial mismatch between theoretical magnetic field models extrapolated from photospheric magnetograms and stereoscopically triangulated loops, in the order of a 3D misalignment angle of $\alpha_{\text {mis }} \approx 20^{\circ}-40^{\circ}$ (DeRosa et al., 2009; Sandman et al., 2009). A comparison of a force-free field model with STEREO-triangulated loops is shown in Figure 11. Some parameterization of the theoretical field models is required to minimize the mismatch. Such a parameterization was implemented in potential-field models in terms of unipolar magnetic charges (Aschwanden and Sandman, 2010) or magnetic dipoles (Sandman and Aschwanden, 2011), which enabled to improve the misalignment angle to $\alpha_{\text {mis }} \approx 10^{\circ}-20^{\circ}$.

Living Reviews in Solar Physics

http://www. livingreviews . org/lrsp-2011-5 


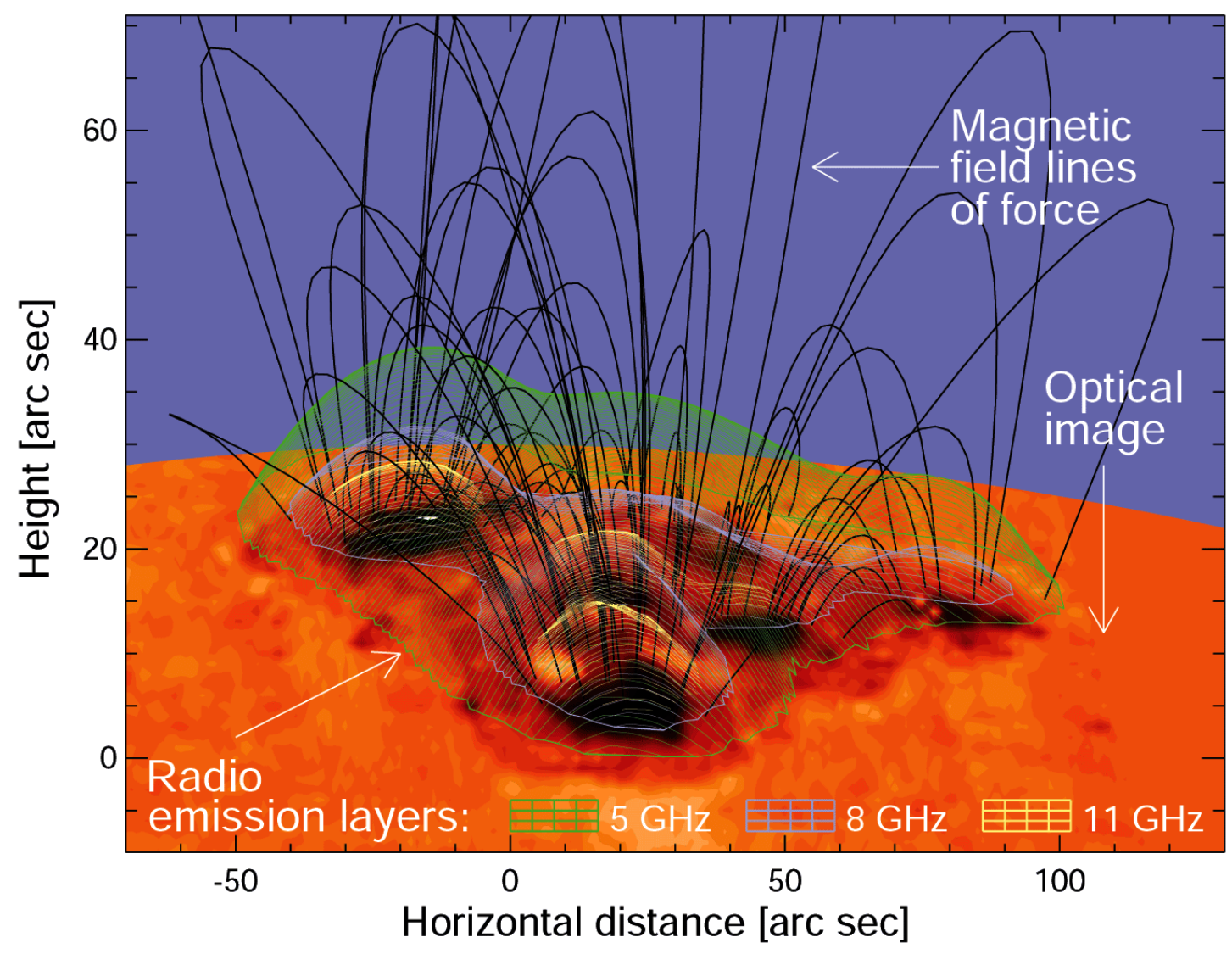

Figure 10: A 3D magnetic field representation is rendered from photospheric magnetograms (optical image in orange), from extrapolated magnetic field lines (black lines), and the iso-Gauss contours for three gyroresonant layers that correspond to gyrofrequencies of $5 \mathrm{GHz}$ (green), $8 \mathrm{GHz}$ (blue), and $11 \mathrm{GHz}$ (yellow). The outer contours of each iso-Gauss surface demarcate the extent of radio emission at each frequency (courtesy of Stephen White and Jeong Woo Lee). 


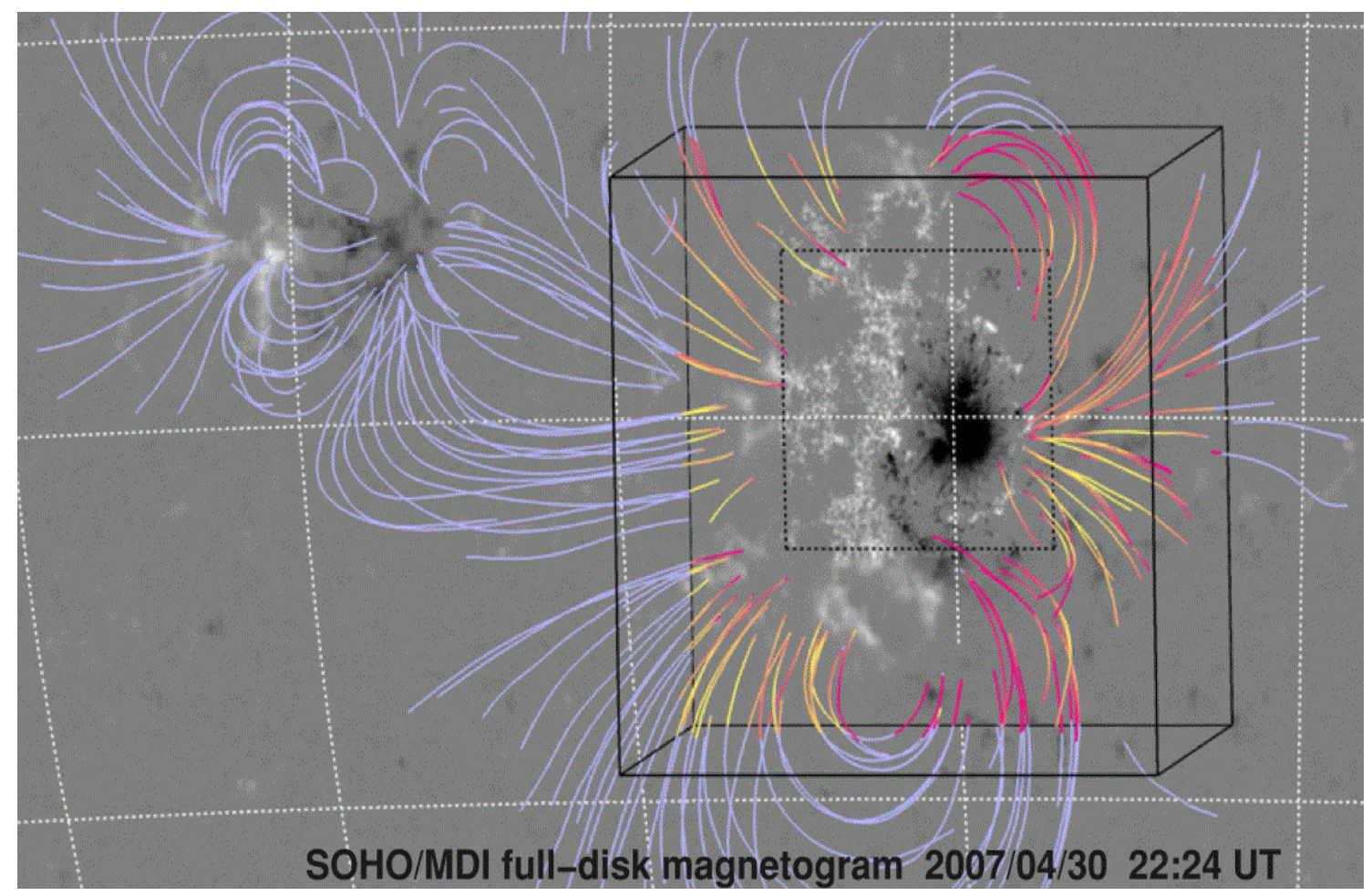

Figure 11: SOHO/MDI magnetogram observed on 2007 Apr 30, 22:42 UT, with overlayed stereoscopically triangulated loop tracings (purple) and calculated NLFFF magnetic field model (colors in central box), where the colors indicate an increasing degree of misalignment from $\alpha_{\text {mis }}<5^{\circ}$ (yellow) to $\alpha_{\text {mis }}>45^{\circ}$ (red) (from DeRosa et al., 2009). 

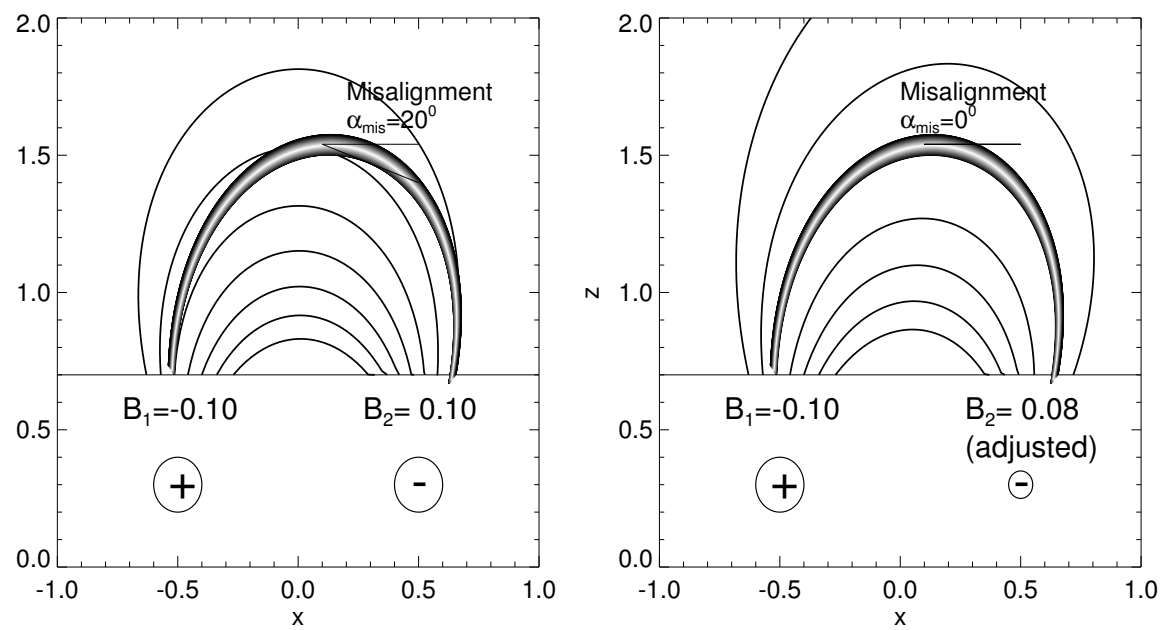

Figure 12: Left: A potential dipole field is calculated with two unipolar magnetic charges buried in equal depth and equal magnetic field strength, but opposite magnetic polarity $\left(B_{1}=-0.1, B_{2}=+0.1\right)$. An asymmetric EUV loop is observed at the same location (grey torus) with a misalignment of $\alpha_{\text {mis }}=20^{\circ}$ at the top of the loop. Right: The magnetic field strength of the right-hand side unipolar charge is adjusted (to $B_{2}=+0.08$ ) so that the misalignment of the loop reaches a minimum $\left(\alpha_{\text {mis }}=0^{\circ}\right.$ ) (from Aschwanden and Sandman, 2010).

The principle is illustrated in Figure 12. A caveat of these first attempts to adjust theoretical magnetic field models to observed 3D loop coordinates (obtained from stereoscopic triangulation) is that the optimization algorithm takes only the coronal misalignment into account, but neglects the constraints of the observed photospheric magnetograms. The justification for this neglect is the fact that the magnetic field in the photosphere and lower chromosphere is not force-free (Metcalf et al., 1995), but ultimately we would like to have magnetic field models that can handle both the non force-free extrapolation in the chromosphere plus fitting of the force-free field in the corona to observed 2D projections or 3D triangulations of loops. Once we have an optimized (matching) coronal 3D magnetic field model, we can populate each field line with a magnetic flux tube and perform hydrodynamic modeling of entire coronal sections, constrained by the observed EUV and soft $\mathrm{X}$-ray images in different temperature filters, a technique called instant stereoscopic tomography of active region (ISTAR) (Aschwanden et al., 2009c).

\subsection{Stereoscopic tomography and 3D forward-fitting}

We turn now to "true tomographic methods", which require simultaneous observations from multiple aspect angles, in contrast to the "pseudo-tomographic methods" using the solar rotation to vary the aspect angle over time as we described in Section 3.2. One of the simplest methods is the backprojection method (Figure 4). The principle of the backprojection method in tomographic reconstruction, as used in medical tomography, was first demonstrated for a solar image (from Skylab) using 80 projections (Davila and Thompson, 1992). The application to a multispacecraft configuration was then simulated (Figure 13), which demonstrated that a high fidelity of 3D reconstruction can already be achieved for a small number of $(\gtrsim 4)$ spacecraft (Davila, 1994, 1996, 1998). In reality, however, we have only two spacecraft with a stereoscopic view since the launch of STEREO, or three if we combine with a near-Earth spacecraft such as SOHO or SDO. Three viewpoints are not enough to permit tomographic reconstruction with classical methods, 


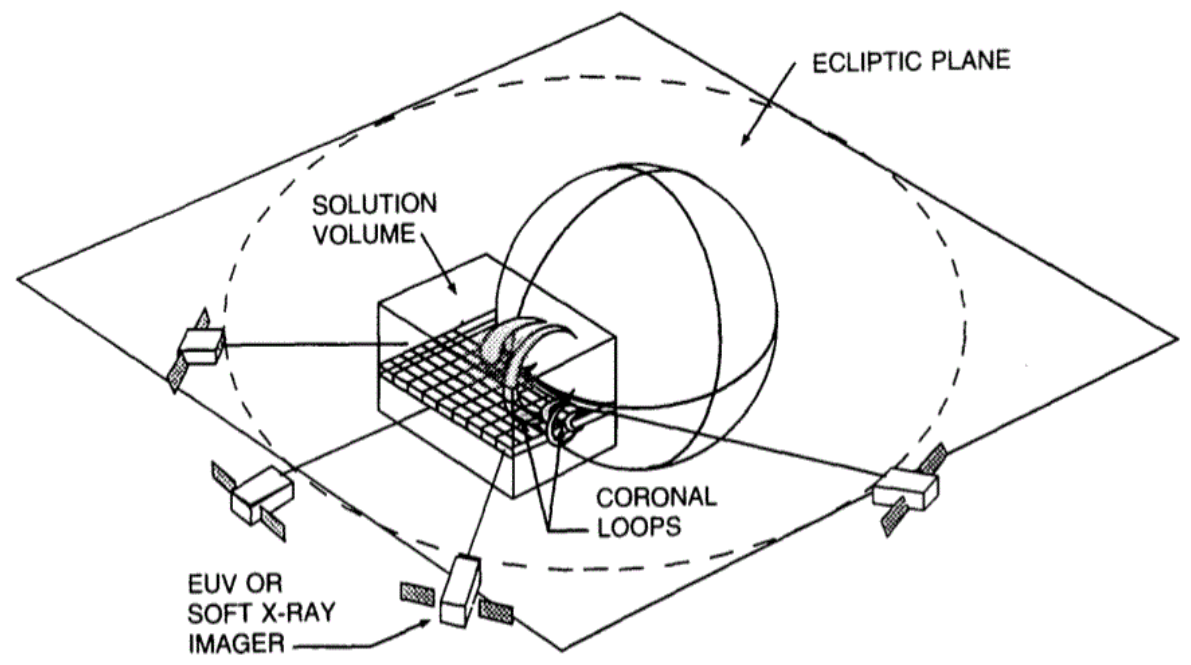

Figure 13: The basic tomography concept of solar tomography is visualized for multiple spacecraft located at different aspect angles from the Sun, which can reconstruct the 2D density distribution in the solar corona (for instance, in the ecliptic plane) by synthesizing the $1 \mathrm{D}$ brightness distributions observed from each spacecraft (from Davila, 1994).

but adding some a priori information on the geometry of a structure may allow to determine the density structure with only three viewpoints (Frazin et al., 2009a).

One a priori information for tomography is a magnetic field model, possibly constrained by stereoscopic triangulation, as we described in the previous section on magnetic stereoscopy. The subset of loops (or loop segments) than could be triangulated in an active region, serves then as a skeleton, while the magnetic field model can fill in an arbitrary set of auxiliary field lines to fill the entire coronal volume. In the case where no fitting theoretical magnetic field line can be found (or be trusted), auxiliary field lines can also be generated by 3D interpolation (Aschwanden et al., 2009c), although this method works only well for a sufficient number of reliably triangulated skeleton field lines, and may contain unphysical solutions that do not fulfill the Maxwell equation of divergencefreeness $(\nabla \cdot \mathbf{B}=0)$ in underpopulated regions. Once a full 3D magnetic field is established for some coronal volume, each field line $[x(s), y(s), z(s)]$ can be populated with a hydrodynamic loop model of the electron temperature $T_{e}(s)$ and electron density $n_{e}(s)$. The emission measure $d E M(T) / d T$ can then be integrated along each line-of-sight $z$, convolved with a filter response function $R(T)$ (Eqs. (4) and (5)), and EUV and soft X-ray images can be rendered for arbitrary instrument filters (Figure 14). Such simulations of coronal images with rendering for particular instrument filters were already simulated in the pre-STEREO era (Gary, 1997; Alexander et al., 1998) and forward-fitted to observed coronal images (Schrijver et al., 2004; Mok et al., 2005; Lundquist et al., 2008a,b), as well as to image pairs from STEREO in three wavelength filters (Aschwanden et al., 2009c).

Alternative methods of 3D reconstruction methods of coronal structures that use auxiliary information involve the line-of-sight velocity measurements of plasma flows in flare loops (Nitta et al., 1999) or in coronal loops (Alissandrakis et al., 2008), time-evolving tomographic 3D reconstruction of polar plumes (Barbey et al., 2008), and multiscale optical-flow methods applied to erupting filaments (Gissot et al., 2008). 


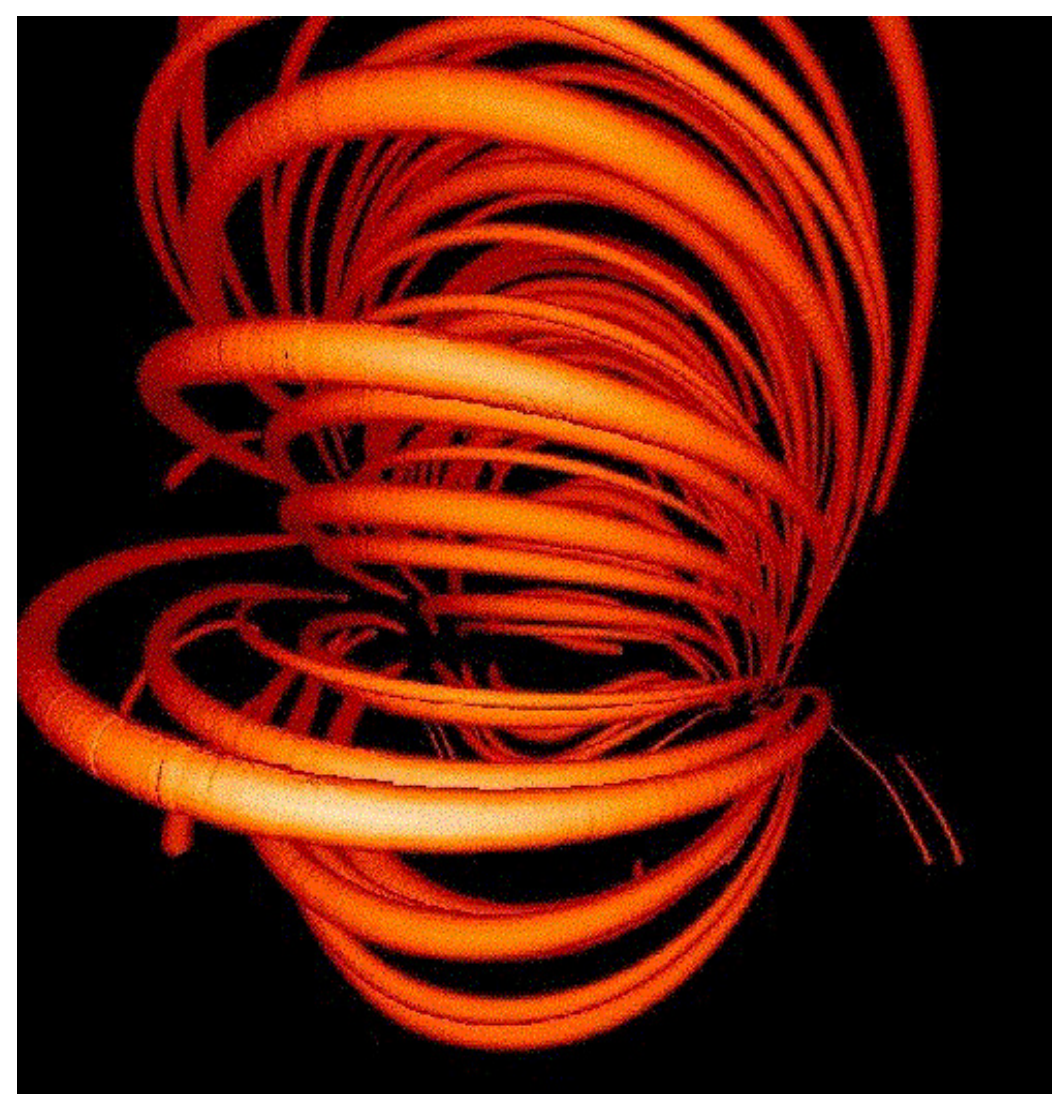

Figure 14: Rendering of solid flux tubes computed with a potential magnetic field model and hydrodynamic 1D models for each loop. The rendering displays a 3D view by shading, but can be scaled to electron densities, temperatures, or brightness in a particular temperature filter (from Gary, 1997). 


\section{Stereoscopic and Tomographic Observations}

In this section we describe the application of stereoscopic and tomographic methods to a variety of coronal observations, grouped by phenomena, with emphasis on observational and physical results, while the technical details of the methods are referred to the previous Section 3.
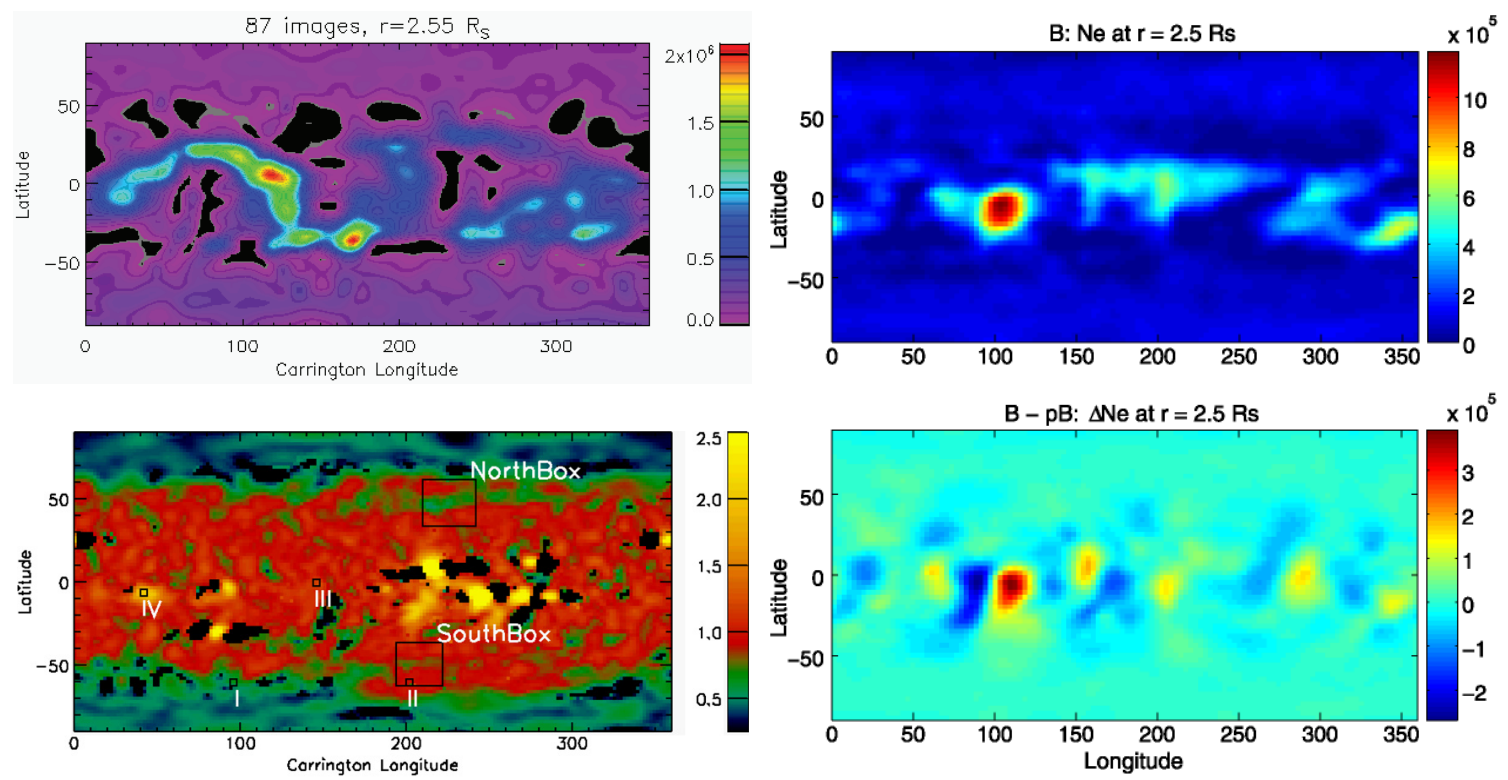

Figure 15: Examples of tomographic reconstructions of the solar corona, visualized as synoptic density maps at a specific height level. Top left: Synoptic map of coronal density at a height of $r=2.55 R_{\odot}$, reconstructed with $87 \mathrm{pB}$ images from LASCO C-2 (Frazin et al., 2007); Bottom left: Synoptic electron density map at a heigth of $r=1.075 R_{\odot}$, reconstructed with a DEM method and STEREO/A and B observations (Frazin et al., 2009b); Right: Density reconstruction $n_{e}(l, b)$ at $r=2.5 R_{\odot}$ from LASCO C-2 total brightness images $B$ (top right) and density difference $\Delta n_{e}(l, b)$ after subtraction of polarized brightness images, $B-p B$, at the same height level (bottom right) (from Frazin et al., 2010).

\subsection{D reconstruction of the large-scale corona}

Reconstructing the solar corona as a whole yields mostly information on the 3D density distribution $n_{e}(l, b, r)$ that varies horizontally (as a function of heliographic longitude $l$ and latitude $b$ ) and vertically (as a function of height $h=r-R_{\odot}$ in the lower corona, say within a few solar radii). The horizontal variations demarcate coronal holes (magnetic open-field regions), quiet-Sun regions (large-scale closed-field regions), active regions (medium-scale closed-field regions containing the strongest magnetic fields), or streamers (quasi-steady structures with bipolar feets and vertical outflows along open field lines, feeding the slow solar wind component). In the solar minimum corona, active regions may disappear completely. Some structures are extremely long-lived, such as coronal holes that survive from 7 up to 27 solar rotations (Abramenko et al., 2010). The vertical coronal structure is mostly governed by gravitational stratification, but tomographic reconstructions can quantify hydrostatic equilibrium versus super-hydrostatic dynamical states. Generally, the $3 \mathrm{D}$ density $n_{e}(l, b, r)$ and temperature distributions $T_{e}(l, b, r)$ obtained from tomographic reconstructions of the full corona contain valuable information on the hydrodynamic structure, heating requirement, magnetic structure, sources of the solar wind, and the heliospheric connectivity.

Living Reviews in Solar Physics

http://www . livingreviews .org/lrsp-2011-5 


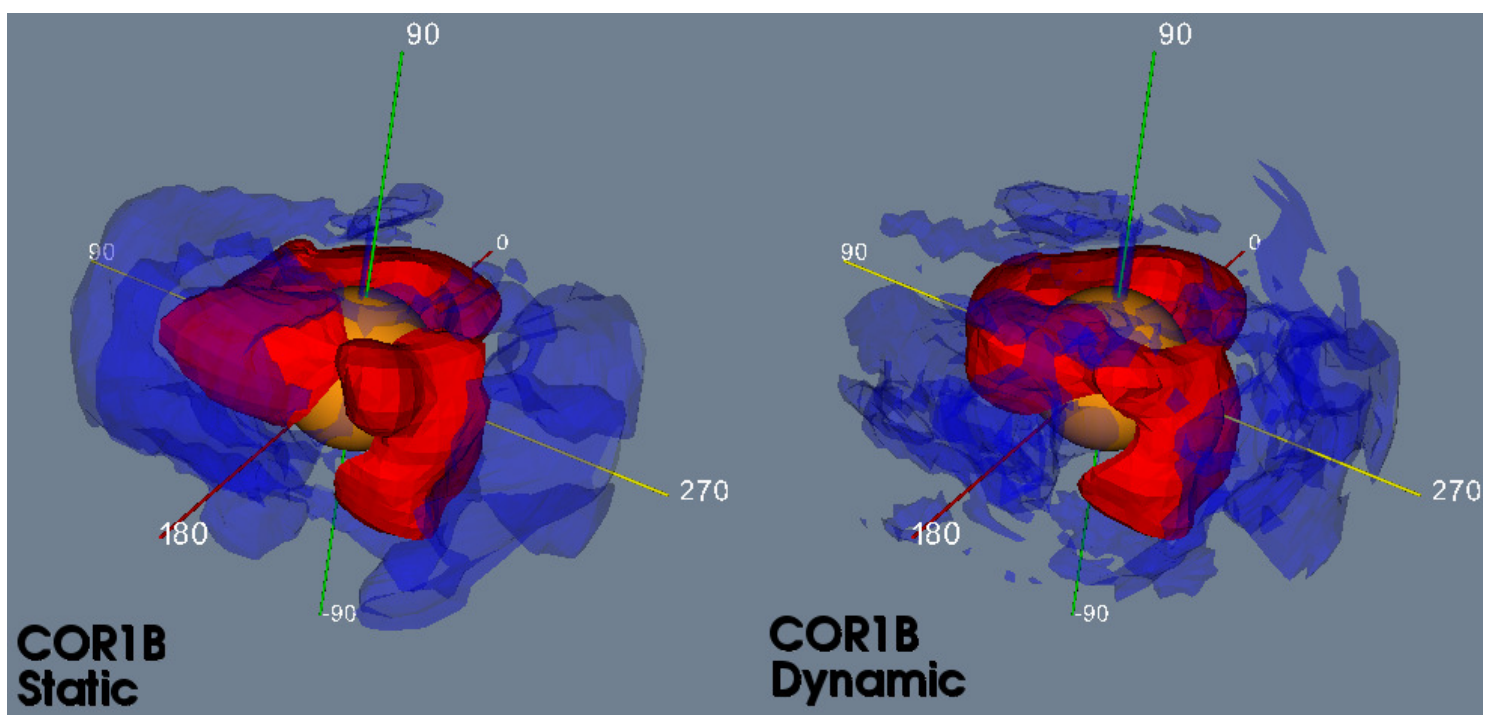

Figure 16: $3 \mathrm{D}$ isosurfaces of electron-density reconstructions of the solar corona using STEREO COR-1 data with a static (left) and a dynamic (right) tomographic reconstruction method (from Butala et al., 2010).

The most restricting constraint of coronal tomography is the static assumption. First attempts of dynamic tomography of the 3D density reconstruction in the solar corona (Frazin et al., 2005a), using a linear time-variability term (similar to Kalman filters), was carried out by Butala et al. (2010) (Figure 16), applied to STEREO COR-1 data at heights of $1.3-4 R_{\odot}$ over a 4 -week period, which yielded a better fit than static solutions.

Because of the under-constraintness of 3D tomographic inversion, assumptions on the global geometry are usually made, such as: (i) spherical symmetry, which yields only the radial density function $n_{e}(r)$; (ii) axis-symmetry, which yields in addition the latitudinal variation $n_{e}(b, r)$ (Quémerais and Lamy, 2002), or (iii) local symmetry with respect to the neutral magnetic surface (e.g., heliospheric current sheet), which can be computed from PFSS magnetic fields (Saez et al., 2007). Polarized brightness (pB) images, such as from LASCO C-2, were initially preferred for tomographic reconstructions, but improved calibrations allowed also the use of total brightness (B) images, after correcting for the weighting function of Thompson scattering (Frazin et al., 2010) (Figure 15, right-hand panels). The radial density profile $n_{e}(h)$, reconstructed with tomographic inversion from Mark III K-coronagraph and LASCO C-1 data during the solar minimum, was found to correspond to scale-height temperatures of $\lambda=1.3-1.9 \mathrm{MK}$ (Zidowitz, 1999), which could be, in the absence of temperature information, either hydrostatic or super-hydrostatic. Using temperature information from multiple soft X-ray filters of Yohkoh and an inversion based on axis-symmetry, the radial electron density scale height $\lambda_{n}$ was found to agree with the effective temperature scale height $\lambda_{T}$ for most locations, except for streamers where the ratio was found to increase up to $\lambda_{n} / \lambda_{T} \lesssim 2.3$, which corresponds to a super-hydrostatic dynamic state (Aschwanden and Acton, 2001). Integrating differential emission measure (DEM) $d E M / d T$ modeling into solar-rotation based tomography, aided by multiple spacecraft (STEREO/A and B) observations, produced density $n_{e}(l, b, h)$ maps at temperatures of $T_{e} \approx 0.5-2.5 \mathrm{MK}$ and in altitude ranges of $r=1.075 R_{\odot}$ (Frazin et al., 2009b) (Figure 15, bottom left) and $r=1.03-1.23 R_{\odot}$ (Vásquez et al., 2010) (Figure 17), (Vásquez et al., 2011) (Figure 18), including polar crown filaments, coronal cavities, and streamers (Vásquez et al., 2009). 



Figure 17: Synoptic maps of solar minimum corona tomographically reconstructed using solar rotation and the dual STEREO/A and B spacecraft, at a height of $r=1.075 R_{\odot}$, showing the electron density $n_{e}$ (top), the (emission measure weighted) temperature $T_{m}$ (middle), and the temperature spread $w_{T}$ (bottom), overlaid with iso-Gauss magnetic field contours $B$ obtained from PFSS models at the same height. The boundaries between open and closed magnetic field domains are marked with black curves (from Vásquez et al., 2010).

Living Reviews in Solar Physics

http://www. livingreviews.org/lrsp-2011-5 


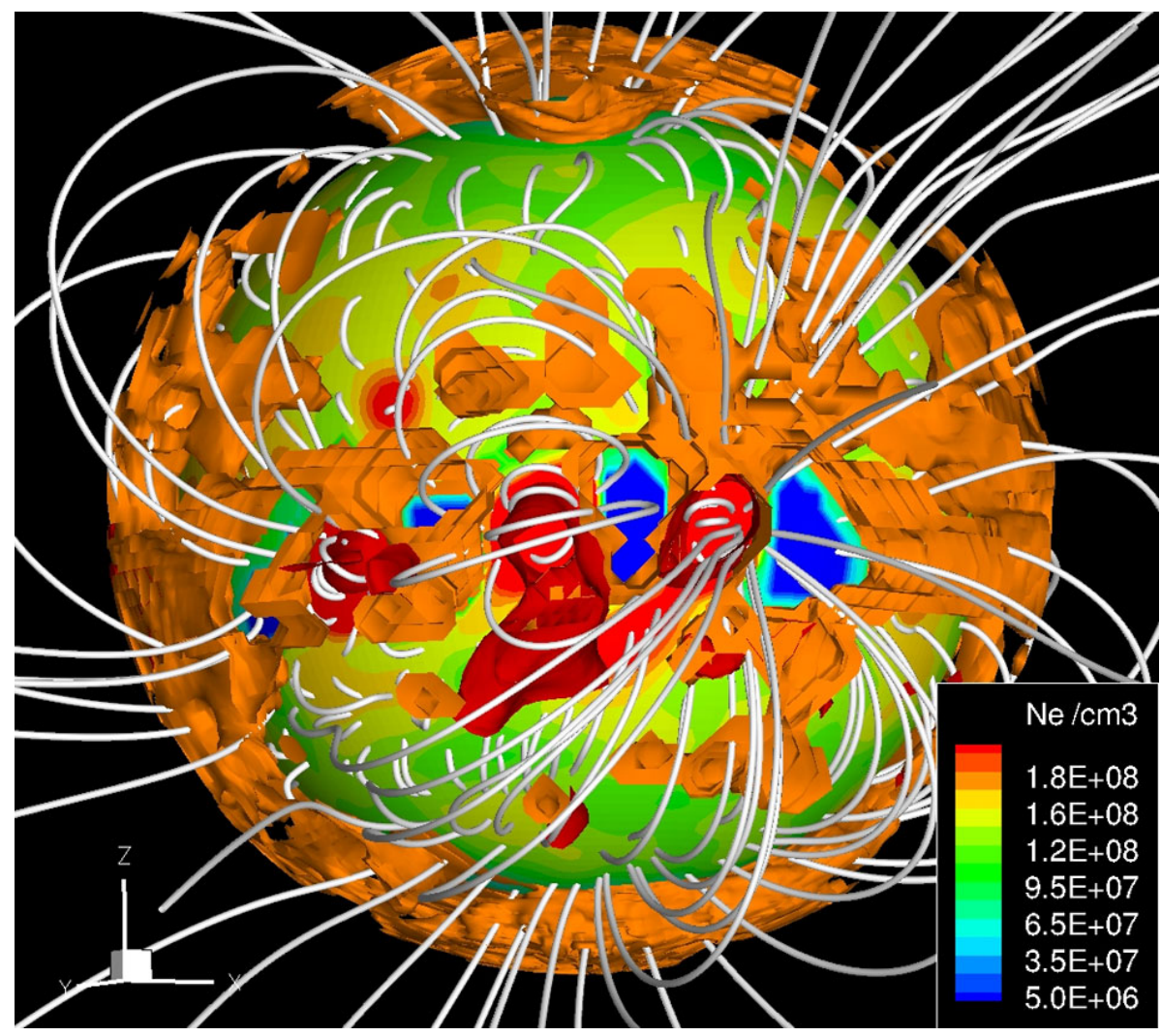

Figure 18: A 3D view of a coronal density and magnetic field reconstruction, showing PFSS magnetic field lines (white), temperature iso-Gauss surfaces (red for $T_{m}=2.0 \mathrm{MK}$ and orange for $T_{m}=1.0 \mathrm{MK}$ ), and density iso-surfaces (see color scale at bottom right) (from Vásquez et al., 2011).

\subsection{Coronal streamers}

The first coronal tomographic reconstructions of Altschuler (1979) from Skylab data visualized a streamer belt map from a polar viewing angle. Tomographic inversion of Mark III K-coronameter data of the minimum corona revealed pronounced longitudinal density variations in the streamer belt (Zidowitz, 1999). Using a higher cadence of 4 polarized brightness (pB) images per day from LASCO C2, instead of 1 pB image per day, the quality of tomographic reconstruction could be dramatically improved (Frazin et al., 2007), especially at a height of $2.55 R_{\odot}$ that serves as a reference level of magnetic potential-field source surface (PFSS) models (Figure 15, top left). 3D reconstructions of coronal streamers from LASCO images at $2.5 R_{\odot}$ were compared with PFSS magnetic field lines and were found to coincide closely with the heliospheric current sheet, often associated with strong magnetic field active regions (Liewer et al., 2001). Based on this it was concluded that many of the bright streamers are the result of scattering from regions of enhanced density associated with active region outflows, and not a result of line-of-sight viewing through folds in a warped current sheet with uniform density (Liewer et al., 2001). However, more detailed tomography with LASCO data revealed double plasma sheets and triple current sheets that are not reproduced by standard PFSS extrapolations (Saez et al., 2005) (Figure 19, top left), visible also in pseudo-tomography maps that subtract a radial gradient filter but do not preserve the electron density (Morgan et al., 2009; Morgan and Habbal, 2010) (Figure 19, right). True tomography of the 3D density distribution with two simultaneous spacecraft observations (STEREO/A and B, 
COR-1) was first performed by Kramar et al. (2009) (Figure 19, bottom left), which demonstrated that the equatorial streamer belt is largely consistent with the variation of the current sheet derived from magnetic potential field models.
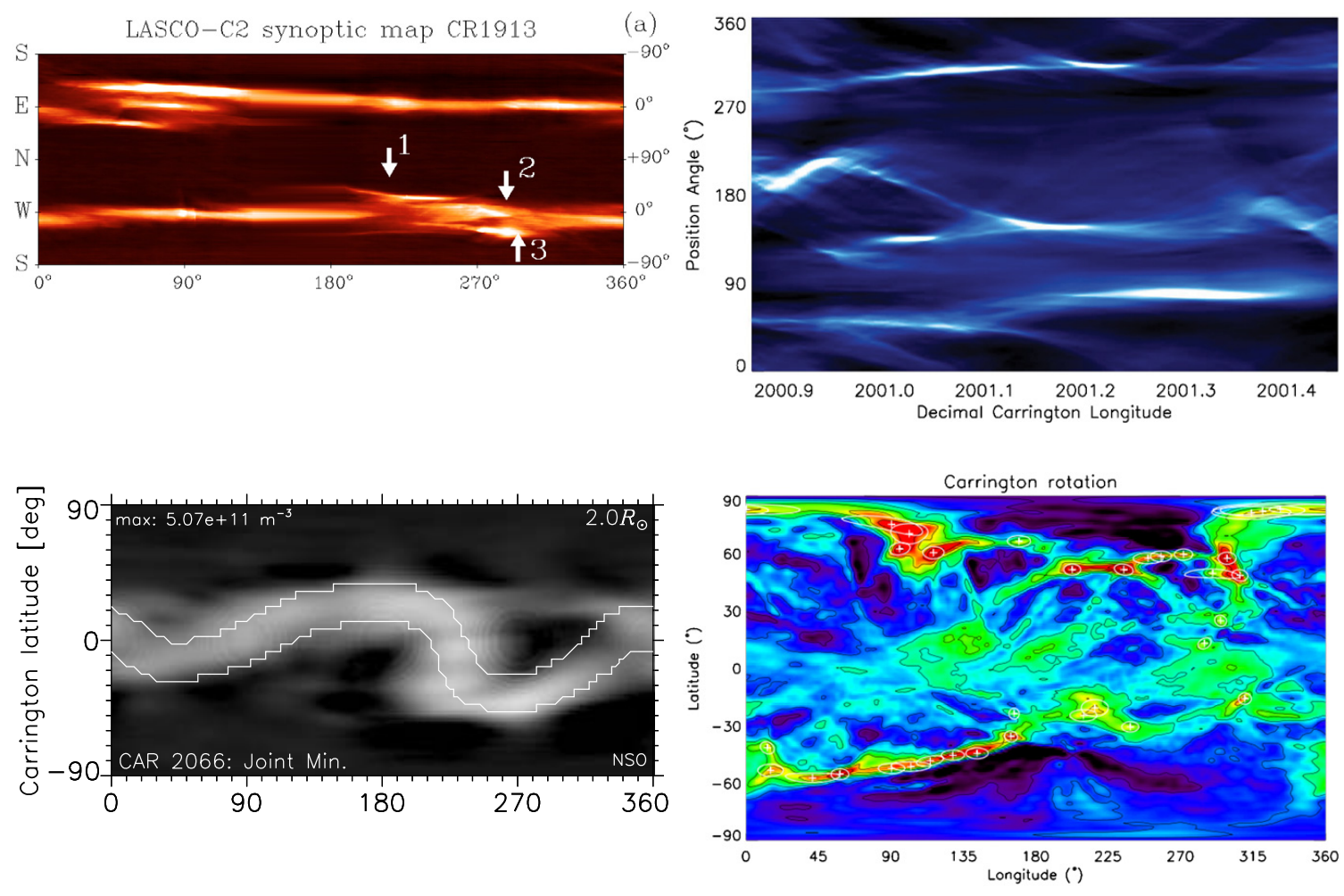

Figure 19: Examples of tomographic streamer belt reconstructions. Top left: Streamer belt density reconstruction with LASCO C-2 at $r=2.5 R_{\odot}$ (Saez et al., 2005); Bottom left: Electron density at height $r=2.0 R_{\odot}$ reconstructed from STEREO COR-1, overlaid with magnetic field contours from NSO/GONG (Kramar et al., 2009); Right: Qualitative density maps at $r=4.0 R_{\odot}$ reconstructed from LASCO C-2 data (Morgan et al., 2009) (top), (Morgan and Habbal, 2010) (bottom).

While streamers were mostly considered as static objects in tomographic reconstruction, dynamic phenomena were also tracked with stereoscopic triangulation, such as streamer blobs that gradually expand outward (Sheeley Jr et al., 2009), and become swept up and compressed by the fast solar wind from low-latitude coronal holes (Sheeley Jr and Rouillard, 2010), or slow streamerblowout CMEs that are considered as a natural consequence of the corona's adjustment to the long term evolutionary driving of the photospheric magnetic field (Lynch et al., 2010). The thermodynamics and kinematics of coronal streamers was also simulated with resistive MHD models and PFSS magnetic models, which allowed the authors to test steady-state flows versus polytropic solutions (Airapetian et al., 2011).

\subsection{Active regions}

Active regions have typical sizes in the range of 10-100 $\mathrm{Mm}$ and, thus, are barely resolved in the coarse-meshed tomographic reconstructions of the (large-scale) solar corona, which explains the lack of active region tomography in literature. However, 3D reconstructions of active regions were attempted with stereoscopic methods in radio wavelengths (Aschwanden and Bastian, 1994a,b;

Living Reviews in Solar Physics

http://www. livingreviews.org/lrsp-2011-5 
Aschwanden, 1995) and EUV (Aschwanden et al., 1999, 2000, 2009c). Imaging of active regions at a wavelength of $\lambda=20 \mathrm{~cm}(\nu=1.5 \mathrm{GHz})$ with the Very Large Array (VLA) was used over 6 different days and 66 Gaussian radio source components from 22 different active regions were stereoscopically triangulated. A height distribution of $h=25 \pm 15 \mathrm{Mm}$ was determined, as well as a systematic center-limb darkening as a function of the center-to-limb angle $\alpha$ was found,

$$
T_{B}(\alpha)=T_{B}(0)\left[0.4+0.6 \cos ^{2}(\alpha)\right],
$$

which was interpreted as an opacity effect of thermal free-free absorption due to denser cool coronal plasma along the line-of-sight near the limb (Aschwanden and Bastian, 1994b; Aschwanden, 1995). Thus, radio stereoscopy can provide quantitative information on the 3D density and temperature distribution of active regions, but the stereoscopically triangulated altitude $h(\lambda)$ of a radio source at a given wavelength localizes only the layer where free-free emission becomes optically thick, which requires an "onion shell-like" parameterization of 3D density and temperature models.

The 3D architecture of an active region can be assembled by modules, consisting of 1D coronal loops. Their 3D coordinates can be calculated by stereoscopic triangulation, either using a solar rotation based method (Section 3.1), as it was applied to SOHO/EIT images (Aschwanden et al., 1999, 2000), or using stereoscopic triangulation (Section 3.3) with the dual STEREO spacecraft (Aschwanden et al., 2009c; Aschwanden and Wülser, 2011; Rodriguez et al., 2009). A review on the $3 \mathrm{D}$ reconstruction, the $3 \mathrm{D}$ geometry, and the $3 \mathrm{D}$ distributions of physical parameters in active regions is given in Aschwanden and Wülser (2011).

Active region NOAA 7986 was reconstructed this way from SOHO/EIT 171, 191, and $284 \AA$ images, and it was found that (i) the loops in the temperature range of $T_{e} \approx 1.0-2.5 \mathrm{MK}$ were in hydrostatic equilibrium (since the expected temperature scale height matched the observed density scale height), and (ii) radiative loss exceeded the conductive loss rate by two orders of magnitude, in contrast to the standard steady-state Rosner-Tucker-Vaiana (RTV) model (Aschwanden et al., 2000). This observation represented the first statistical evidence that EUV loops are dominated by radiative cooling, and that the energy balance postulated by the RTV law (with a constant uniform heating rate) is violated.

Active region NOAA 10955 was observed with STEREO on 2007 May 9 and reconstructed in detail, using stereoscopic triangulation with EUVI STEREO/A and B that provided the 3D geometry $[x(s), y(s), z(s)]$ of some 70 loops in the three temperature filters 171,191 , and $284 \AA$ (Aschwanden et al., 2008c), density $n_{e}(s)$ and temperature $T_{e}(s)$ measurements of these loops (Aschwanden et al., 2008b), which were then synthesized and interpolated into a space-filling 3D model of the density $n_{e}(x, y, z)$ and temperature $T_{e}(x, y, z)$ distribution of the active region (Aschwanden et al., 2009c). A projection of the 3D density and temperature distributions is shown in Figure 20. The density and temperature solutions for each of the 8000 modular loops are not unique, of course, but are constrained by a dual set of three temperature filter images and, thus, should at least closely represent the statistical distribution of the active region. Interestingly, the full-loop modeling includes also extrapolated temperatures to the loop footpoints (apexes) that are cooler (hotter) than the EUVI filter temperature range of $T_{e} \approx 1.0-2.5 \mathrm{MK}$, and this way the differential emission measure (DEM) distribution of the active region could be reconstructed in the full temperature range of $\log (T)=5.0-7.0$ (Figure 21).

The forward-fitting of parameterized loop density $n_{e}(s)$ and temperature $T_{e}(s)$ profiles in this study yielded also statistics on the hydrostaticity of the active region loops. The statistical dependence of the pressure scale height $\lambda_{p}\left(T_{m}\right)$ on the loop apex temperature $T_{m}$ revealed mostly super-hydrostatic scale heights for cool EUV loops $\left(T_{m} \approx 0.5-3.0 \mathrm{MK}\right)$, while the hotter $\left(T_{m} \approx 3-6 \mathrm{MK}\right)$ soft X-ray emitting loops were found to be slightly below the expected hydrostatic scale height (Figure 22). This means that the heating rate approximately balances the conductive cooling rate in soft X-rays (as expected in the steady-state energy balance RTV model), while the 

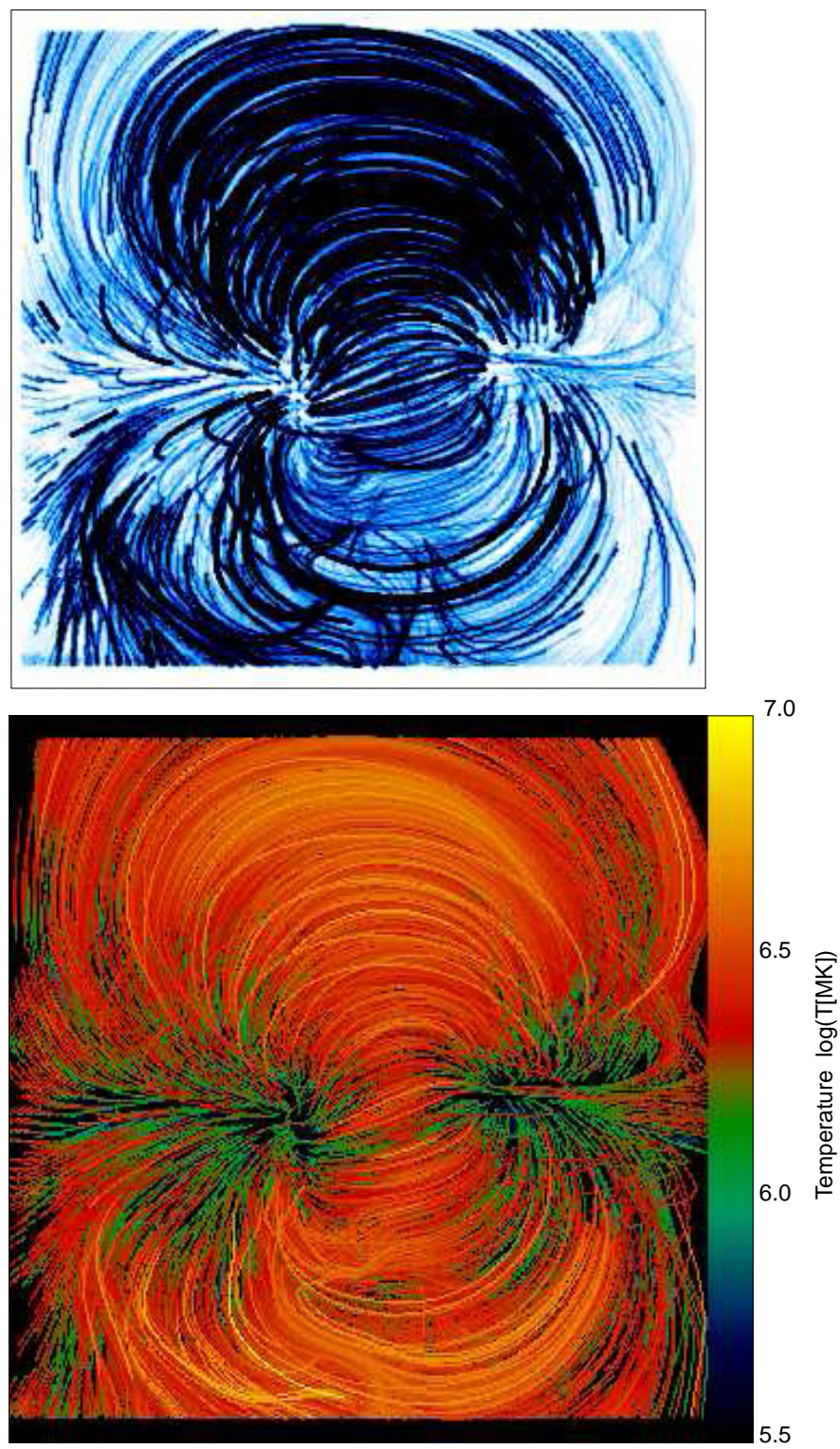

Figure 20: Density map (top) and temperature map (bottom) of active region NOAA AR 10955 observed on 2007 May 9 with STEREO/EUVI and reconstructed with the ISTAR method (Instant Stereoscopic Tomography of Active Regions). The model contains some 8000 loop components, of which a skeleton of 70 loops has been stereoscopically triangulated (from Aschwanden et al., 2009c). 


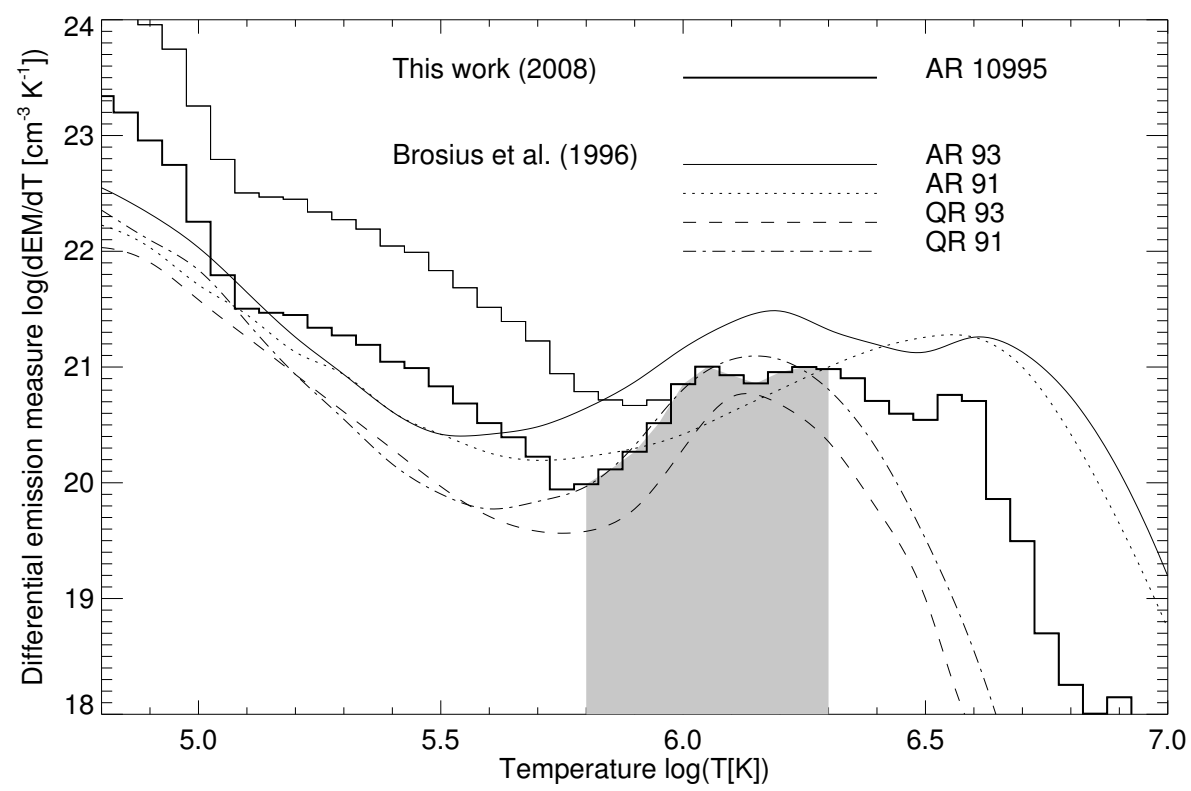

Figure 21: The differential emission measure distribution $\operatorname{dEM}(\mathrm{T}) / \mathrm{dT}$ is computed from the ISTAR tomographic model and is compared with two active regions and two quiet-Sun regions from Brosius et al. (1996). Note that the primary temperature sensitivity range of EUVI is $\log (T) \approx 5.8-6.3$ (grey range), but the DEM could be constrained in the range of $\log (T) \approx 5-7$, based on the parameterized temperature profiles used in the stereoscopic tomography code. A canopy correction is applied in the temperature range of $\log (T)=5.7-6.0$, with a quadratic area expansion from $10 \%$ to $100 \%$ of the coronal flux tube area (histogram with thick linestyle). The uncorrected DEM is also shown (upper histogram) (from Aschwanden et al., 2009c).

radiative loss rate dominates the heating rate in the cooler EUV loops. In other words, soft X-ray loops are close to steady-state, while EUV loops are in non-equilibrium.

Extended temperature analysis of active regions with Hinode data (Noglik et al., 2009; Rodriguez et al., 2009) and AIA/SDO (Aschwanden and Boerner, 2011; Aschwanden et al., 2011) with a comprehensive set of temperature filters in the entire range of $T_{e} \approx 0.5-16 \mathrm{MK}$ reveals the basic temperature structure of active regions quite clearly: The hottest loops are found in the compact core of the active region, which straddle the neutral line, have a relatively small length scale, and emit in soft X-rays, while the cooler loops overarch the active region, have relatively large length scales, and emit in EUV. This tells us also something about the heating rate, which is the lower per volume element, the longer the loops are. Thus, stereoscopic and tomographic $3 \mathrm{D}$ reconstruction of active regions provide important information on the hydrostaticity, the energy balance between heating and cooling, and this way offer a sensitive diagnostic of the coronal heating process.

\subsection{Coronal loops}

Coronal loops essentially represent curvi-linear 1D structures with a small transverse cross-section and a noticable density contrast to the background corona, so that their 3D geometry can be triangulated with stereoscopy (Section 3.3). Stereoscopy of coronal loops is pursued for at least three important reasons in coronal physics: (i) The 3D geometry measures the inclination angle of the average loop plane, which is a necessary parameter to derive the vertical pressure scale height; 


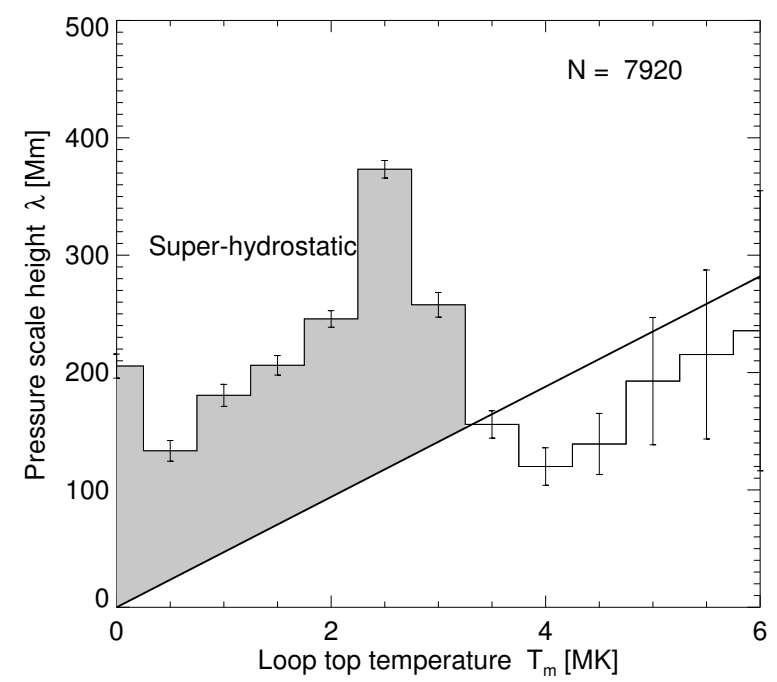

Figure 22: Average pressure scale heights $\lambda_{n}$ as a function of the loop apex temperature $T_{m}$. The error bars indicate the error of the mean value $e_{m}=\sigma / \sqrt{(N)}$, with $\sigma$ being the standard deviation. For comparison, the linear relationship between the pressure scale height and temperature for hydrostatic equilibrium is also shown (thick line), i.e., $\lambda_{T}=47(\mathrm{~T} / 1 \mathrm{MK}) \mathrm{Mm}$. Note that EUV loops with apex temperatures of $T_{m} \lesssim 3 \mathrm{MK}$ show a trend to be super-hydrostatic, while the soft X-ray loops with $T_{m} \approx$ $3-6 \mathrm{Mm}$ approximately follow the hydrostatic equilibrium (from Aschwanden et al., 2009c).

(ii) The 3D geometry measures the loop length and the local line-of-sight angles, which are key parameters for hydrodynamic flux tube modeling and related scaling laws; (iii) The 3D geometry is the most important observational constraint to test theoretical magnetic field models of the solar corona, and it also reveals whether loops follow a magnetic closed-field or open-field line. We will discuss these physical aspects in the context of stereoscopic observations in turn.

\subsubsection{Hydrostatic scale height of loops}

The $3 \mathrm{D}$ loop geometry can be parameterized by a set of $3 \mathrm{D}$ coordinates $\left[x_{i}, y_{i}, z_{i}\right]$, where the projected positions $\left[x_{i}, y_{i}\right]$ are directly measured in the image plane, and the distance $z_{i}$ is calculated via stereoscopy (Section 3.3). If we approximate the 3D loop geometry with a semi-circular shape, we can characterize it with 6 parameters: the loop curvature center position $\left(x_{c}, y_{c}, z_{c}\right)$, the loop curvature radius $r_{c}$, the azimuth angle of the footpoint baseline $\alpha$, and the inclination angle $\theta$ of the loop plane to the vertical, where the latter two angles are defined in a heliographic coordinate system (Figure 23).

The pressure scale height in a hydrostatic (gravitationally stratified) atmosphere depends on the height $h$ above the surface, which can be specified as a function of the loop length coordinate $s$, e.g., for a vertical semi-circular loop as

$$
h(s)=r(s)-R_{\odot}=\frac{2 L}{\pi} \sin \left(\frac{\pi s}{2 L}\right)
$$

where $L$ is the loop half length. The hydrodynamic pressure $p(s)$ in a vertical semi-circular loop is then, based on the pressure balance or momentum equation, taking only gravity into account 


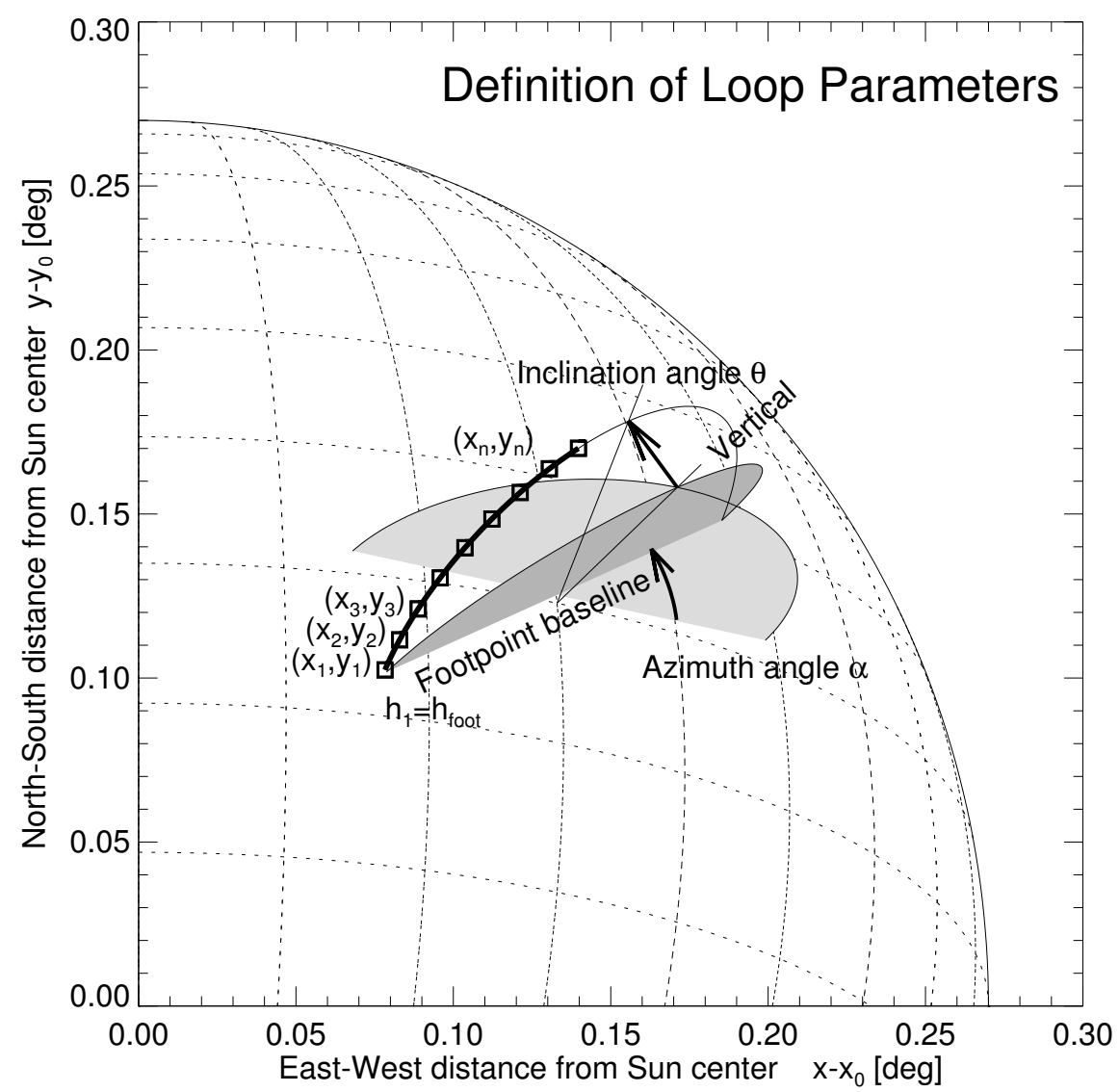

Figure 23: Definition of loop parameters: loop point positions $\left(x_{i}, y_{i}\right), i=1, \ldots, n$ starting at the primary footpoint at height $h_{1}=h_{\text {foot }}$, the azimuth angle $\alpha$ between the loop footpoint baseline and heliographic east-west direction, and the inclination angle $\theta$ between the loop plane and the vertical to the solar surface.

(Aschwanden, 2005),

$$
p(s)=p_{0} \exp \left[-\frac{\left(h[s]-h_{0}\right)}{\lambda_{p}\left(T_{e}\right)\left(1+\frac{h[s]}{R_{\odot}}\right)}\right] \approx p_{0} \exp \left[-\frac{\left(h[s]-h_{0}\right)}{\lambda_{p}\left(T_{e}\right)}\right],
$$

with $\lambda_{p}\left(T_{e}\right)$ being the vertical pressure scale height,

$$
\lambda_{p}\left(T_{e}\right)=\frac{2 k_{B} T_{e}}{\mu m_{H} g_{\odot}} \approx 4.7 \times 10^{9}\left(\frac{T_{e}}{1 \mathrm{MK}}\right) \quad(\mathrm{cm}) .
$$

Note that we use the so-called coronal approximation $T_{e}=T_{i}$ for the coronal temperature throughout this paper, although differences may exist in non-collisional regimes in the upper corona.

However, if the loop plane is inclined by an inclination angle $\theta$ with respect to the local vertical direction on the solar surface, the observed scale height $\lambda_{p}^{\text {obs }}$ as derived from the exponential density drop along the loop, has to be corrected by the cosine of the inclination angle,

$$
\lambda_{p}^{\mathrm{obs}}=\frac{\lambda_{p}}{\cos \theta} .
$$


This can be understood also in terms of communicating water tubes where the water level depends only on the height, but not on the actual length of an inclined water tube segment (Figure 24). This is the reason why $3 \mathrm{D}$ stereoscopy is important for the understanding of the hydrostatics of a coronal plasma loop. If a semi-circular loop has a curvature radius of $r_{c} \approx 100 \mathrm{Mm}$, which approximately corresponds to two density or pressure scale heights at a temperature of $T=1.0 \mathrm{MK}$ (Eq. (27)), the electron density at the loop top is a factor of $e^{-2}=0.14$ lower than at the footpoint, which makes the EUV brightness $\left(I \propto n_{e}^{2}\right)$ drop by a factor of $e^{-4}=0.02$, while the loop apex can be almost equally bright as the loop footpoints for a highly inclined loop $(\theta \gtrsim 0)$. As a consequence, vertical loops are typically only visible in the lowest scale height, while highly inclined loops are visible over their entire length. An example of a stereoscopic 3D reconstruction of 70 loops in an active region using STEREO/A and B is shown in Figure 25, which illustrates that the vertical range of detected loop segments does not exceed about one pressure scale height (see height projection above limb, Figure 25 top left), and that complete loops are all highly inclined (see horizontal projection and side view in Figure 25 bottom left and right).
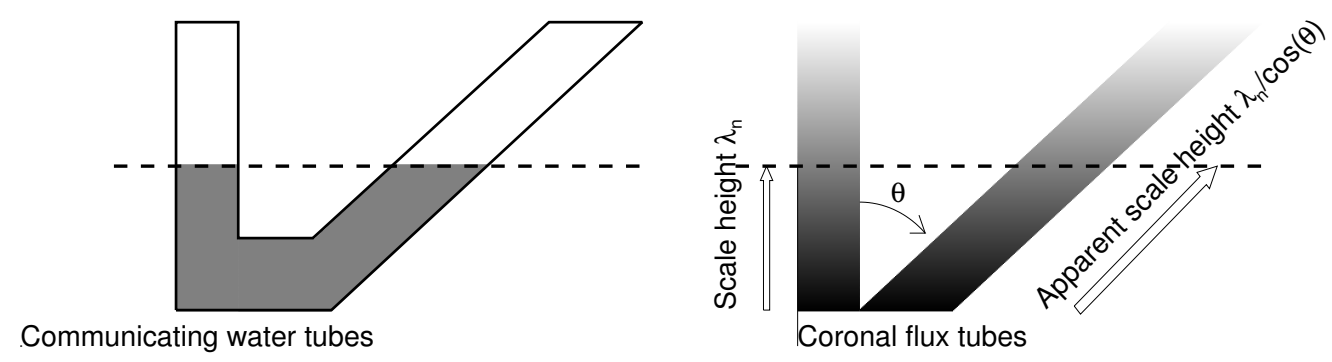

Figure 24: Vertical and apparent density scale heights in coronal loops (right) and analogy with communicating water tubes (left) (from Aschwanden, 2005).

First determinations of the 3D geometry of individual coronal loops have been carried out using Skylab data and the solar rotation (Berton and Sakurai, 1985), finding loop asymmetry, non-planar geometry, and mean inclination angles of $\theta \approx 7^{\circ}-25^{\circ}$. This study demonstrated the feasibility of $3 \mathrm{D}$ reconstruction, the ability to measure deviations from simple circular and planar loop geometries, but worked only for very large (inter-active region) loops due to the static restriction for solar rotation-based methods. Similarly, the 3D geometry of 65 coronal loops has been triangulated using EIT images and the solar rotation, yielding loop lengths of $2 L \approx 300-800 \mathrm{Mm}$, loop heights of $h \approx 70-330 \mathrm{Mm}$, and inclination angles in a range of $|\theta| \lesssim 40^{\circ}$ (Aschwanden et al., 1999, 2000). Using parameterized 3D geometry models, non-planar loop geometries can even be determined from forward-fitting to observed loop projections in a single-spacecraft image without stereoscopy, as demonstrated for twisted helical geometries using EIT images (Portier-Fozzani et al., 2001; Portier-Fozzani and Inhester, 2001).

The first true stereoscopic triangulations of loops have been performed with the dual STEREO/A and B spacecraft (Feng et al., 2007a; Aschwanden et al., 2008c). Feng et al. (2007a) fitted the 3D shape of 5 loops with a linear force-free magnetic field model and could constrain the twist of the loops in term of the nonlinear force-free $\alpha$-parameter. Aschwanden et al. (2008c) was able to determine the non-planarity $\left(|\Delta y| / r_{c} \approx 3 \%-11 \%\right)$, the non-circularity $\left(|\Delta r| / r_{c} \approx 11 \%-30 \%\right)$, and inclination $\left(\theta \approx 35^{\circ}-73^{\circ}\right)$ in 7 complete loops. One problem of solar loop triangulation is the incompleteness of tracable loop segments due to confusion in crowded locations. This problem is particularly present in automated loop detection algorithms, for instance using the loop segmentation method (Inhester et al., 2008) or the Oriented Coronal CUrved Loop Tracing (OCCULT) code (Aschwanden, 2010).

Living Reviews in Solar Physics

http://www. livingreviews.org/lrsp-2011-5 


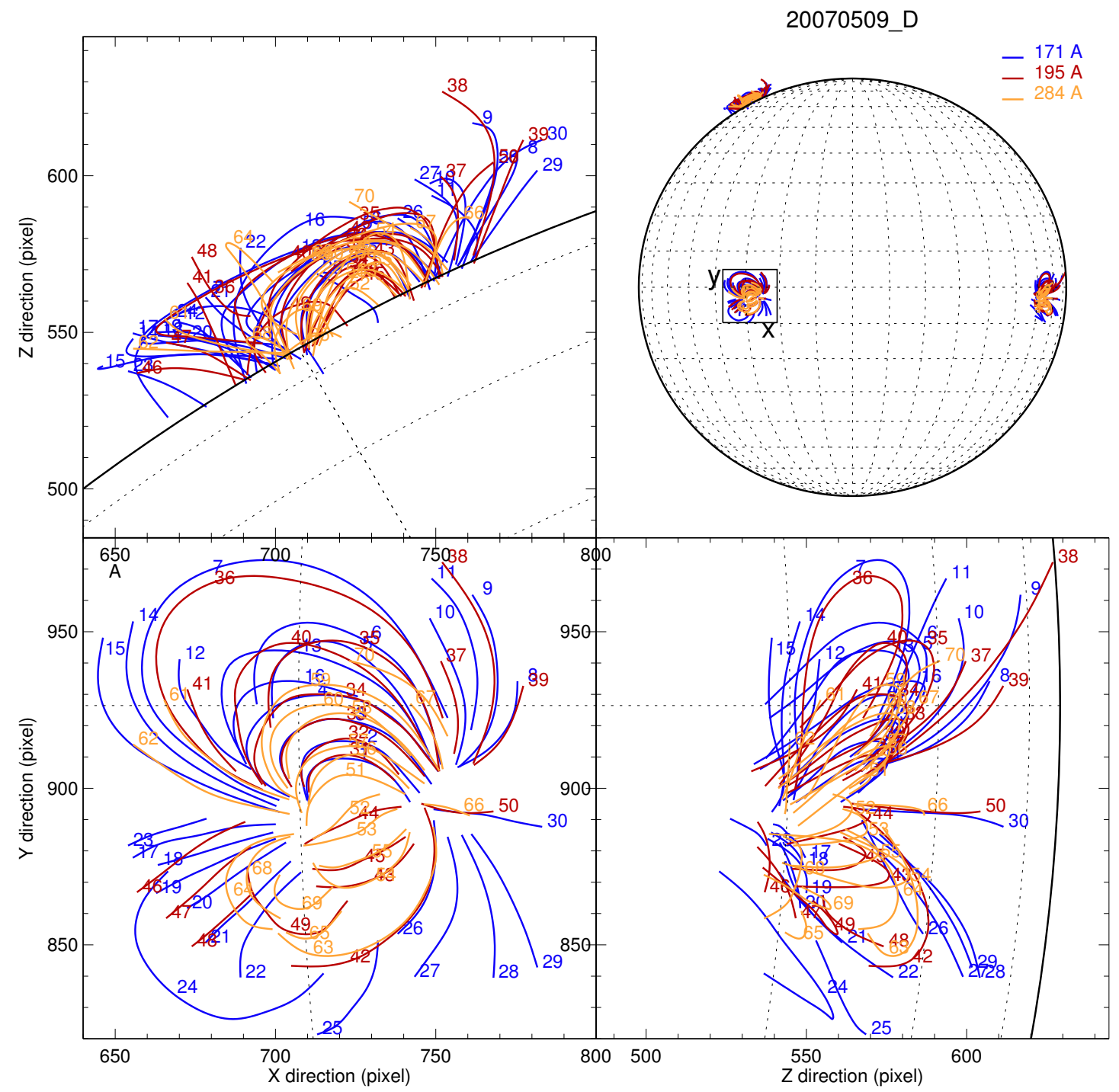

Figure 25: Orthogonal projections of the stereoscopically triangulated 70 coronal loops in AR 10955 observed on 2007 May 9 in three filters (171 $\AA=$ blue; $195 \AA=$ red; $284 \AA$ = yellow). The observed projection in the $x-y$ image plane seen from spacecraft $\mathrm{A}$ is shown in the bottom panel left, the projection into the $x-z$ plane in the top panel left, and the projection into the $y-z$ plane in the bottom panel right. The three orthogonal projections correspond to rotations by $90^{\circ}$ to the north or west (to positions indicated on the solar sphere in the top right panel) (from Aschwanden et al., 2009c). 


\subsubsection{Hydrodynamics of loops}

1D hydrodynamic loop models can be parameterized by the electron density $n_{e}(s, t)$ and temperature evolution $T_{e}(s, t)$ as a function of the loop length coordinate $s$ and time $t$. Observational measurements of these parameters can be inferred from the soft X-ray and EUV intensities, which are proportional to the squared density (Eqs. (4) and (5)) and the line-of-sight column depth $w_{z}$,

$$
F_{\lambda} \propto \iint n_{e}^{2}(z, T) R_{\lambda}(T) d T d z
$$

which in the case of a 1D flux tube can be reduced to constant values of the local loop density $n_{e}(s)$ and temperature $T_{e}(s)$, integrated over a column depth $w_{z}$, which corresponds to the loop width $w$ divided by the cosine of the local line-of-sight angle $\psi(s)$,

$$
w_{z}\left[x_{i}, y_{i}, z_{i}\right]=\frac{w}{\cos \left(\psi\left[x_{i}, y_{i}, z_{i}\right]\right)}
$$

and can be calculated from the stereoscopically triangulated loop coordinates $[x(s), y(s), z(s)]$,

$$
\cos \left(\psi\left[x_{i}, y_{i}, z_{i}\right]\right)=\frac{\sqrt{\left(x_{i+1}-x_{i}\right)^{2}+\left(y_{i+1}-y_{i}\right)^{2}}}{\sqrt{\left(x_{i+1}-x_{i}\right)^{2}+\left(y_{i+1}-y_{i}\right)^{2}+\left(z_{i+1}-z_{i}\right)^{2}}} .
$$

Thus, stereoscopic measurements of the 3D loop coordinates and background-subtracted EUV and soft X-ray fluxes $F_{\lambda}(s)$ along the loop coordinate $s$ allows us to fully constrain the physical parameters $n_{e}(s)$ and $T_{e}(s)$ of the loop, as well as to measure the loop half length $L$ in $3 \mathrm{D}$ space. Since multiple temperature filters are required, forward-fitting of a hydrodynamic loop model to observed fluxes $F_{\lambda}(s)$ is generally a more viable way than direct inversion. The geometric effect of the column depth integration and scale height correction in inclined loops is illustrated in Figure 26.

Simulations of multiple coronal loops that form an active region based on 1D hydrostatic models (Figure 14) and their 3D reconstruction from stereoscopic images has been discussed and tested in the pre-STEREO era (Gary et al., 1998), including magnetic modeling (Gary and Alexander, 1999). The first stereoscopically constrained inversion of loop density $n_{e}(s)$ and temperature profiles $T_{e}(s)$ has been carried out with STEREO/A and B triple-filter data (Aschwanden et al., 2008b). Although the same parameters can also approximately be inferred from a single spacecraft, as demonstrated with EIT data (Aschwanden et al., 1999, 2000), dual spacecraft stereoscopy yields more accurate values because of a true measurement of the line-of-sight angle, inclination of loop plane, and independent background subtraction from two different aspect angles. The self-consistency between the two independent spacecraft measurements from STEREO/A and B is evident from the obtained ratios of loop temperatures $\left(T_{B} / T_{A}=1.05 \pm 0.09\right)$, densities $\left(n_{B} / n_{A}=0.94 \pm 0.12\right)$, and loop widths $\left(w_{B} / w_{A}=0.96 \pm 0.05\right)$, as shown in Figure 27 (Aschwanden et al., 2008b).

The stereoscopic measurement of the loop half length $L$, the electron density $n_{e}$, and loop apex temperature $T_{e}$ enables us to test $1 \mathrm{D}$ loop scaling laws, such as the RTV scaling law, $p_{\mathrm{RTV}}=$ $\left(T_{e} / 1400\right)^{3} L^{-1}$, by comparing the theoretically predicted pressure $p_{\mathrm{RTV}}$ with the observed pressure $p_{\text {obs }}=2 n_{e} k_{B} T_{e}$. Interestingly, while the RTV law approximately holds for hot soft X-ray emitting loops, it completely fails for EUV loops at cooler temperatures, giving rise to an over-density or over-pressure ratio $q$ of (in cgs-units),

$$
q=\frac{n_{\mathrm{obs}}}{n_{R T V}}=\frac{p_{\mathrm{obs}}}{p_{\mathrm{RTV}}}=\frac{2 n_{e} k_{B} T_{e}}{(1 / L)\left(T_{e} / 1400\right)^{3}}=7.57 \times 10^{-7} \frac{n_{e} L}{T_{e}^{2}} .
$$

This over-pressure is shown for stereoscopically triangulated loops in Figure 28. The overpressure amounts to $q \approx 3-15$, which indicates a strong deviation from the RTV energy balance equilibrium between heating and cooling rates. In the impulsive heating scenario (e.g., Aschwanden

Living Reviews in Solar Physics

http://www. livingreviews.org/lrsp-2011-5 
COLUMN DEPTH
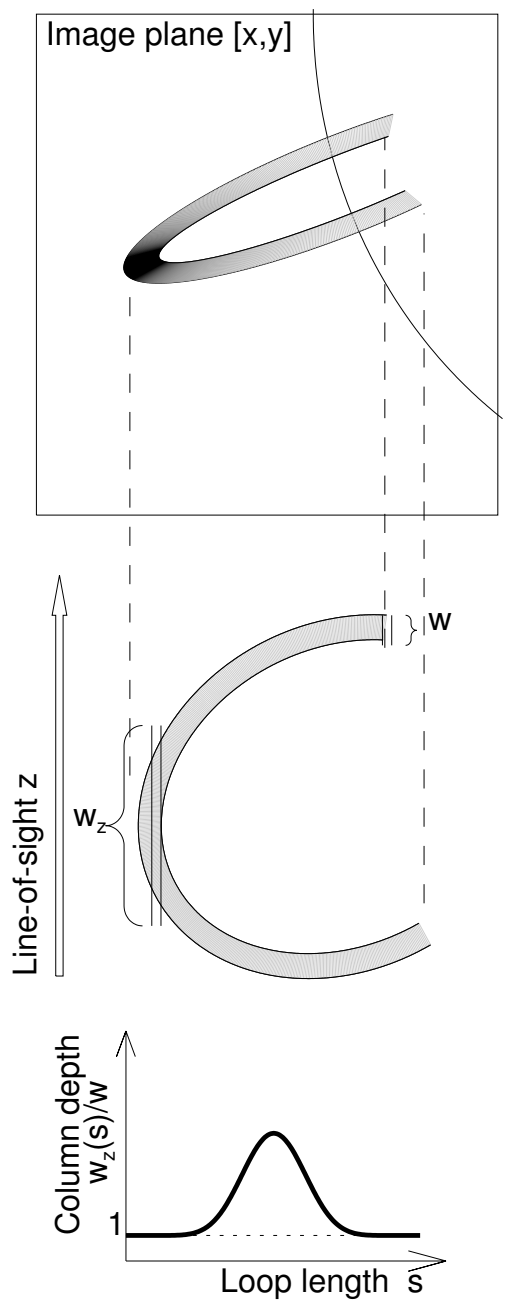

SCALE HEIGHT
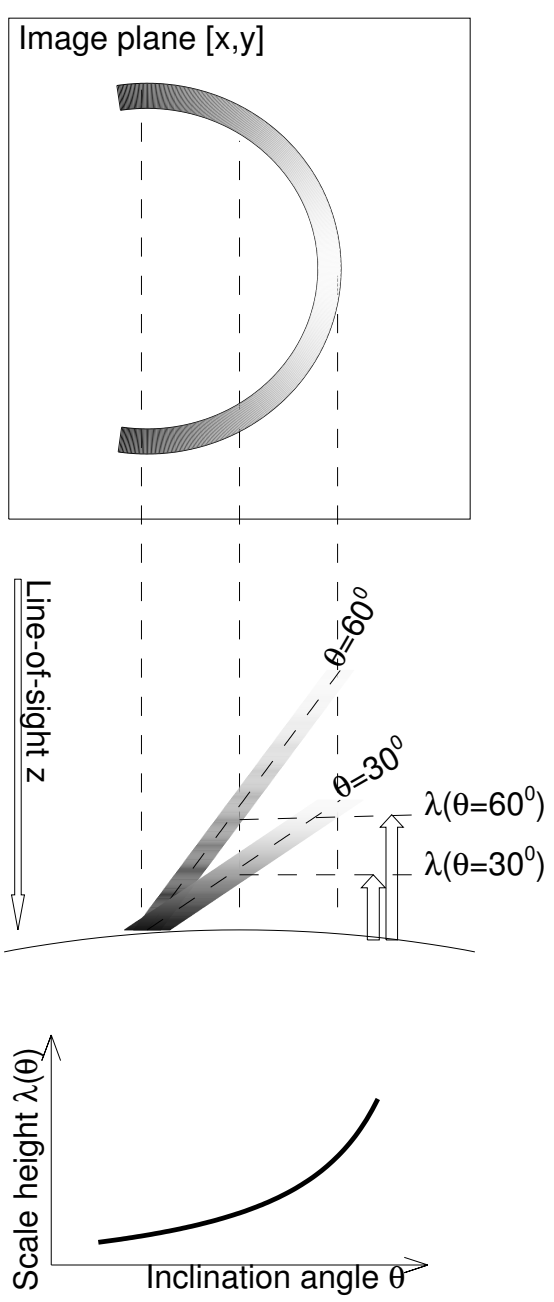

Figure 26: Left panel: the effect of the variable column depth $w_{z}(s)$ measured parallel to the line-of-sight $z$ is illustrated as a function of the loop length parameter $s$, for a loop with a constant diameter $w$. Right panel: the effect of the inclination angle $\theta$ of the loop plane on the inferred density scale height $\lambda(\theta)$ is shown. Both effects have to be accounted for when determining the electron density $n_{e}(s)$ along the loop (from Aschwanden, 2005). 

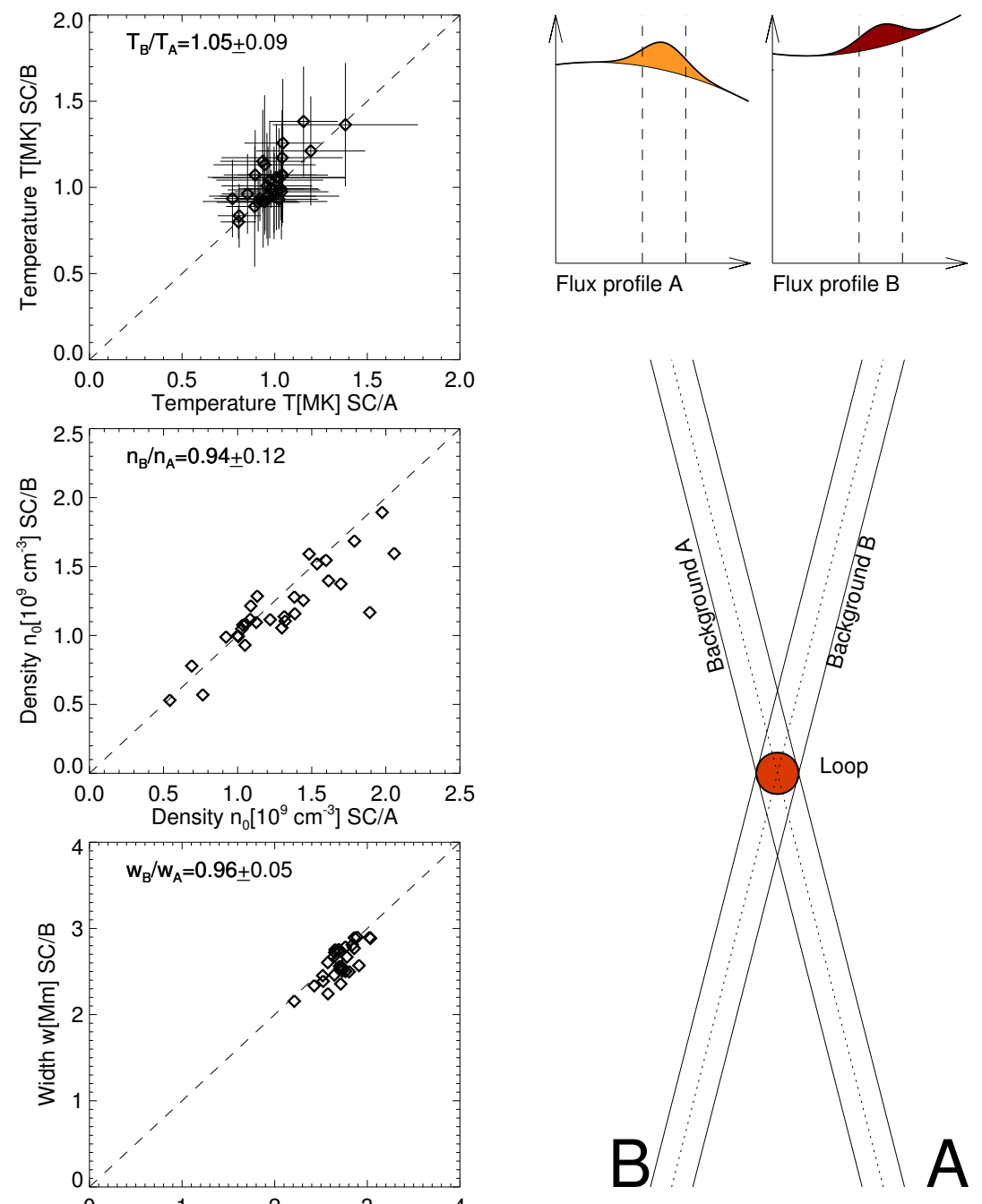

Figure 27: Self-consistency of mean loop temperatures $T_{A}, T_{B}$ (top left), base electron densities $n_{A}, n_{B}$ (middle left), and mean loop widths $w_{A}, w_{B}$ (bottom left) measured with spacecraft STEREO/A vs. STEREO/B. These loop parameters are inferred from the background-subtracted loop-associated flux (top right), based on independent background subtractions for the different line-of-sights of both spacecraf A and B (bottom right) (from Aschwanden et al., 2008b). 
and Tsiklauri, 2009) EUV loops exhibit a higher density than predicted by the RTV steady-state equilibrium (Lenz et al., 1999; Aschwanden et al., 2000, 2008b), once the heating rate fades, in particular in the late cooling phase when the radiative loss rate dominates.

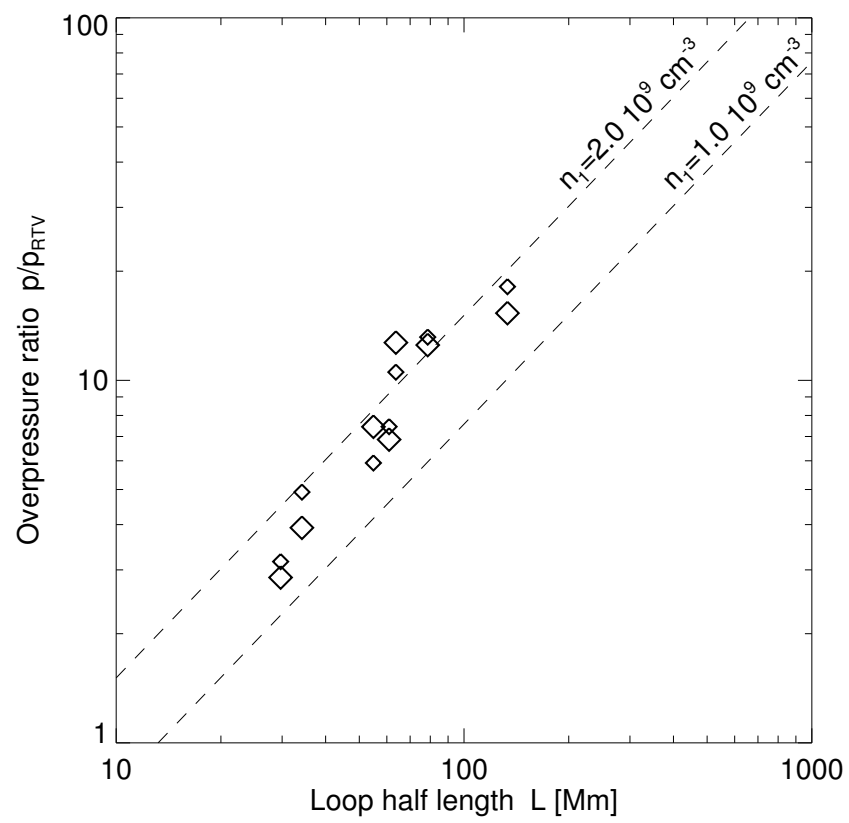

Figure 28: The loop overpressure factor $q_{p}=p / p_{\mathrm{RTV}}$ (normalized by Rosner-Tucker-Vaiana scaling law with uniform heating) is shown versus the loop half length $L$. Datapoints are given for the complete 7 loops detected along their full length, measured with STEREO-A (large diamonds) and with STEREO-B (small diamonds) (from Aschwanden et al., 2008b).

Loops with known density $n_{e}(s)$, temperature $T_{e}(s)$, and width profiles $w(s)$ represent the building blocks of active regions and the solar corona at large and, thus, can be used for tomographic modeling of active regions (Aschwanden, 2009a). Physical properties of cooling plasma in quiescent active region loops, such as its emission measure and filling factor can also be determined using the two line-of-sights of EUVI from STEREO/A and B, in combination with SOHO/SUMER, UVCS, EIT, LASCO, and Hinode (Landi et al., 2009). Direct detections of small-scale siphon flows in funnel-like legs of coronal loops by both STEREO spacecraft were also reported (Tian et al., 2009), as well as cool flows along coronal loops (Zhang and Li, 2009). Quantitative modeling of the hydrodynamic evolution of coronal loops, based on STEREO/EUVI, Hinode/EIS, XRT, and TRACE data, however, still represents a major challenge that cannot easily be reconciled in all observed wavelengths (Warren et al., 2010).

\subsubsection{Magnetic fields of loops}

While we outlined several methods of "magnetic stereoscopy" in Section 3.4, we review here some observational results of stereoscopic magnetic field modeling for coronal loops in more detail.

The simplest magnetic field model is a potential field, which can be characterized by a magnetic scalar potential function $\Phi(\mathbf{r})$,

$$
\mathbf{B}(\mathbf{r})=\nabla \Phi(\mathbf{r}),
$$


which fulfills Maxwell's equation of divergence-freeness,

$$
\nabla \cdot \mathbf{B}=\nabla^{2} \Phi=0
$$

In principle, for a given boundary condition, e.g., from vector magnetograph data $\mathbf{B}(l, b, h=0)$, and ignoring data noise, the solution of a potential magnetic field $\mathbf{B}(\mathbf{r})$ is unique and has no free parameters that can be adjusted to match observed loop geometries.

Widely used non-potential magnetic field models that include currents $\mathbf{j}(\mathbf{r})$, are the force-free models, characterized by an $\alpha$-parameter that is a constant for linear force-free (LFF) models,

$$
(\nabla \times \mathbf{B})=4 \pi \mathbf{j}=\alpha \mathbf{B},
$$

or a spatially varying function $\alpha(\mathbf{r})$ for nonlinear force-free (NLFF) models,

$$
(\nabla \times \mathbf{B})=4 \pi \mathbf{j}=\alpha(\mathbf{r}) \mathbf{B}
$$

Using stereoscopic triangulation, the $3 \mathrm{D}$ coordinates $\mathbf{r}_{\mathrm{obs}}(s)=[x(s), y(s), z(s)]$ of coronal loops can be determined, which can be compared with the $3 \mathrm{D}$ coordinates $\mathbf{r}_{f f}(s, \alpha)=[x(s), y(s), z(s)]$ of the nearest field line of a force-free model, e.g., by varying the $\alpha$-parameter and minimizing the average least-square deviation between the loop coordinates,

$$
\min \left[\Delta_{2}(\alpha)\right]=\frac{1}{s_{\max }^{2}} \int_{0}^{s_{\max }} \sqrt{\left[\mathbf{r}_{\mathrm{obs}}(s)-\mathbf{r}_{f f}(s, \alpha)\right]^{2}} d s .
$$

This method was used by Wiegelmann and Neukirch (2002) and a best-fit value $\alpha$ was determined for the linear force-free model. Thus, the stereoscopic information provides a measurement of the average current (Eq. (35)) for this model,

$$
\mathbf{j}=\frac{\alpha \mathbf{B}}{4 \pi} .
$$

Instead of fitting a constant $\alpha$ for all loops (in the LFF model), each loop could be fitted separately, which may yield an approximative force-free model (NLFF) model, but a self-consistent NLFF solution would require an iteration of a space-filling distribution $\alpha(\mathbf{r})$ that converges to a global solution of Eq. (36).

If a pair of corresponding loops can be unambiguously triangulated with stereoscopy, the obtained 3D coordinates can serve in assessing the accuracy of a theoretical magnetic field model. However, since loop segmentation and identification of corresponding loop pairs are often ambiguous, theoretical magnetic field models can be used to improve stereoscopic triangulation. This method of "magnetic stereoscopy" was proposed by Wiegelmann and Inhester (2006) and Wiegelmann et al. (2009), who applied multiple (potential, linear, and nonlinear force-free) magnetic field models to solve the correspondence problem of stereoscopic loop pairs (by optimization of the criterion Eq. (37)). This method has then been applied to real STEREO data for the first time by Feng et al. (2007a), who found values of $|\alpha| \approx(2-8) \times 10^{-3} \mathrm{Mm}^{-1}$ for 5 loops, which corresponds to a helical twist angle of $\Phi=2 \pi n<0.5$, well below the kink instability threshold $(\Phi \leq 3.5 \pi)$. The same method was also applied to SOHO/EIT and TRACE data using solar rotation stereoscopy (Feng et al., 2007b).

A comprehensive comparison of 11 NLFF and one potential-field model with a set of stereoscopically triangulated coronal loops (Figure 11) was carried out by DeRosa et al. (2009), who find average $3 \mathrm{D}$ misalignment angles in the range of $\alpha_{\text {mis }}=24^{\circ}-44^{\circ}$, which was attributed to three problems: (i) limited area of vector magnetograph data (from Hinode); (ii) uncertainties of boundary data; and (iii) non-force-freeness at photosphere-corona interface. In fact, the NLFF models

Living Reviews in Solar Physics

http://www. livingreviews.org/lrsp-2011-5 
did not fare better than the potential-field model in this case. More cross-comparisons have been performed by Sandman et al. (2009), who finds 3D misalignment angles,

$$
\alpha_{\text {mis }}=\arccos \left(\frac{\mathbf{r}_{\mathrm{obs}} \mathbf{r}_{\mathrm{pot}}}{\left|\mathbf{r}_{\mathrm{obs}}\right| \cdot\left|\mathbf{r}_{\mathrm{pot}}\right|}\right),
$$

of $\alpha_{\text {mis }}=25^{\circ} \pm 8^{\circ}, 19^{\circ} \pm 6^{\circ}$, and $36^{\circ} \pm 13^{\circ}$ for 370 stereoscopically triangulated loops in three active regions observed with STEREO. In a next step it was attempted to forward-fit potential field models with adjustable parameters to bootstrap a best-fit solution, either in terms of $N$ multiple unipolar charges $\mathbf{B}_{j}$ (Aschwanden and Sandman, 2010),

$$
\mathbf{B}(\mathbf{r})=\sum_{j=1}^{N} \mathbf{B}_{j}(\mathbf{r})=\sum_{j=1}^{N} B_{j}\left(\frac{z_{j}}{r_{j}}\right)^{2} \frac{\mathbf{r}_{j}}{r_{j}},
$$

or in terms of a $N$ submerged magnetic dipoles defined by the magnetic moments $\mathbf{m}_{j}$ (Sandman and Aschwanden, 2011),

$$
\mathbf{B}(\mathbf{r})=\sum_{j=1}^{N} \mathbf{B}_{j}(\mathbf{r})=\frac{\mu_{0}}{4 \pi} \sum_{j=1}^{N}\left[\frac{3 \hat{\mathbf{r}}_{j}\left(\hat{\mathbf{r}}_{j} \cdot \mathbf{m}_{j}\right)-\mathbf{m}_{j}}{\left|\mathbf{r}-\mathbf{r}_{j}\right|^{3}}\right],
$$

where $\mathbf{r}_{j}$ is the position of the $j$-th dipole, with $\mathbf{r}$ being the position vector, and $\hat{\mathbf{r}}_{j}=\left(r-r_{j}\right) /\left|r-r_{j}\right|$ the normal vector.

Both potential-field parameterizations achieved a smaller misalignment, which reduced to of $\alpha_{\text {mis }}=16^{\circ} \pm 14^{\circ}, 11^{\circ} \pm 7^{\circ}$, and $18^{\circ} \pm 11^{\circ}$ for the same three active regions in the case of the dipolar model (Sandman and Aschwanden, 2011), and similar values for the case of the unipolar model (Aschwanden and Sandman, 2010). An example is shown in Figure 30, which represents the first of the compared active regions, observed on 2007 Apr 30 (which is identical to the NLFF modeling shown in Figure 11). Since stereoscopic errors are estimated to have uncertainties of similar magnitude $\left(\Delta \alpha_{\mathrm{SE}} \approx 10^{\circ}\right)$, future attempts may envision bootstrapping of stereoscopy with iterative magnetic field variations in order to obtain a best match. A summary of the observed misalignment angles measured in four active regions is shown as a function of the soft X-ray flux in Figure 29 and tabulated in Table 1: The optimized unipolar potential field model (PFU) has about half the misalignment than a standard potential source surface (PFSS) model, and taking the stereoscopic errors into account $\left(\alpha_{\mathrm{SE}}\right)$, the residual misalignment that could be attributed to the non-potentiality of the magnetic field are in the order of $\alpha_{N P}=\sqrt{\alpha_{\mathrm{PFU}}^{2}-\Delta \alpha_{\mathrm{SE}}^{2}} \approx 11^{\circ}-17^{\circ}$. Interestingly, the average misalignment angles are correlated with the soft X-ray flux of the active region (Figure 29), which implies a relationship between electric currents $\mathbf{j}$ and plasma heating (Aschwanden and Sandman, 2010).

Linear force-free (LFF) models of coronal loops in active regions have one free parameter, the twist $\alpha$, which can be constrained by stereoscopic triangulation. Iterating this stereoscopic fitting procedure as a function of time yields then the time evolution of the twist parameter $\alpha(t)$ and nonpotential magnetic energy $E_{\mathrm{LFF}}(t)$, which was found to change significantly during a magnetic flux emergence event (Conlon and Gallagher, 2010). The free (non-potential) energy $E_{\mathrm{NLFF}}$ calculated with a NLFF model with Hinode data was also found to be correlated with the soft X-ray flare index in a sample of 75 active regions (Jing et al., 2010). 


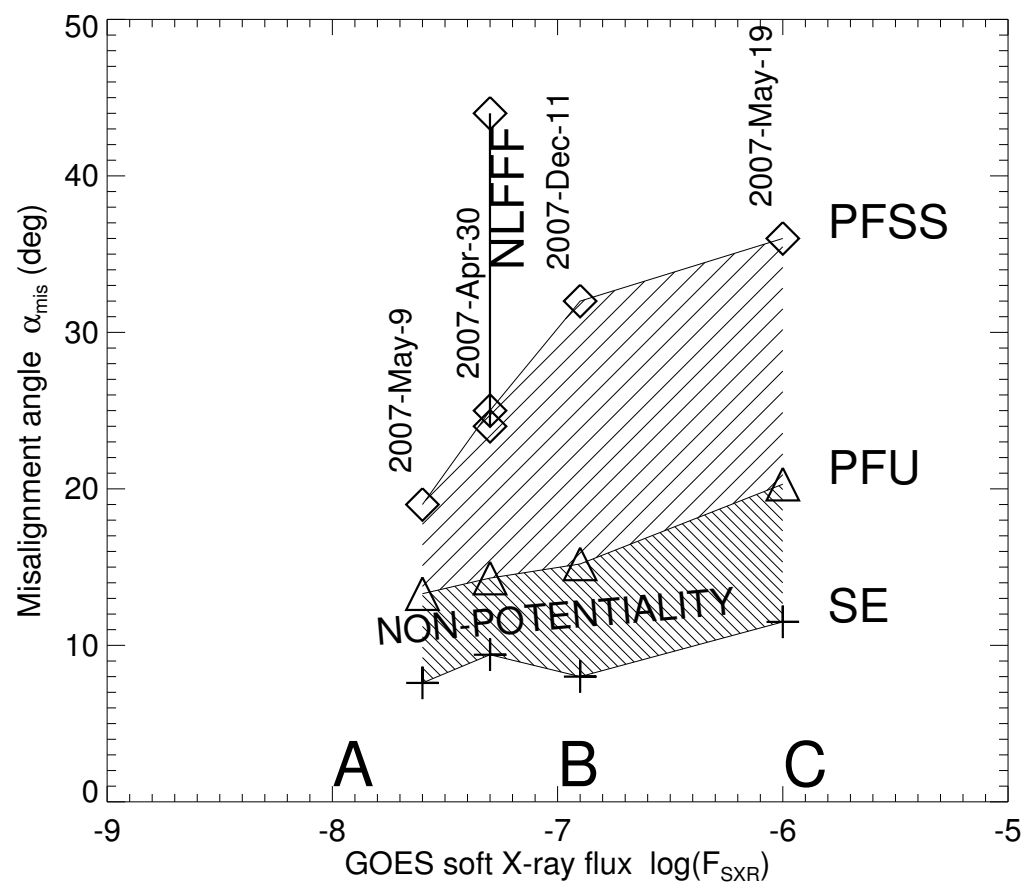

Figure 29: The mean misalignment angle for four active regions as a function of the GOES soft Xray flux: for the potential field source surface model (PFSS: diamonds), for the unipolar potential field model bootstrapped with observed STEREO loops (PFU: triangles), and contributions from stereoscopic measurement errors (SE; crosses). The difference between the best-fit potential field model (triangles) and stereoscopic errors (crosses) can be considered as a measure of the non-potentiality of the active region (hatched area) (from Aschwanden and Sandman, 2010).

Table 1: Misalignment statistics of stereoscopically triangulated loops in four active regions (Aschwanden and Sandman, 2010).

\begin{tabular}{|c|c|c|c|c|}
\hline Parameter & 2007-Apr-30 & 2007-May-9 & 2007-May-19 & 2007-Dec-11 \\
\hline Misalignment $\mathrm{NLFFF}^{1}$ & $24-44$ & & & \\
\hline Misalignment $\mathrm{PFSS}^{2}$ & $25 \pm 8$ & $19 \pm 6$ & $36 \pm 13$ & $32 \pm 10$ \\
\hline Misalignment $\mathrm{PFU}^{3}$ & $14.3 \pm 11.5$ & $13.3 \pm 9.3$ & $20.3 \pm 16.5$ & $15.2 \pm 12.3$ \\
\hline Median $\mathrm{PFU}^{3}$ & 20.0 & 16.2 & 25.8 & 15.7 \\
\hline Stereoscopy error ${ }^{4}$ & 9.4 & 7.6 & 11.5 & 8.9 \\
\hline Non-potentiality $^{5}$ & $11 \pm 9$ & $11 \pm 8$ & $17 \pm 14$ & $12 \pm 10$ \\
\hline GOES soft X-ray flux ${ }^{6}$ & $10^{-7.3}$ & $10^{-7.6}$ & $10^{-6.0}$ & $10^{-6.9}$ \\
\hline GOES class & $\mathrm{A} 7$ & $\mathrm{~A} 4$ & $\mathrm{C} 0$ & B1 \\
\hline
\end{tabular}

1) Measured with nonlinear force-free field code (DeRosa et al., 2009).

${ }^{2}$ ) Measured with potential field source surface code (Sandman et al., 2009).

3 ) Measured with unipolar potential field model (Aschwanden and Sandman, 2010).

4) Measured from inconsistency between adjacent loops.

$\left.{ }^{5}\right)$ Residual misalignment of unipolar best-fit model with stereoscopic error subtracted in quadrature, $\alpha_{\mathrm{NP}}=\sqrt{\alpha_{\mathrm{PFU}}^{2}-\Delta \alpha_{\mathrm{SE}}^{2}}$.

${ }^{6}$ ) GOES flux in units of [ $\left.\mathrm{W} \mathrm{m}^{-2}\right]$. 


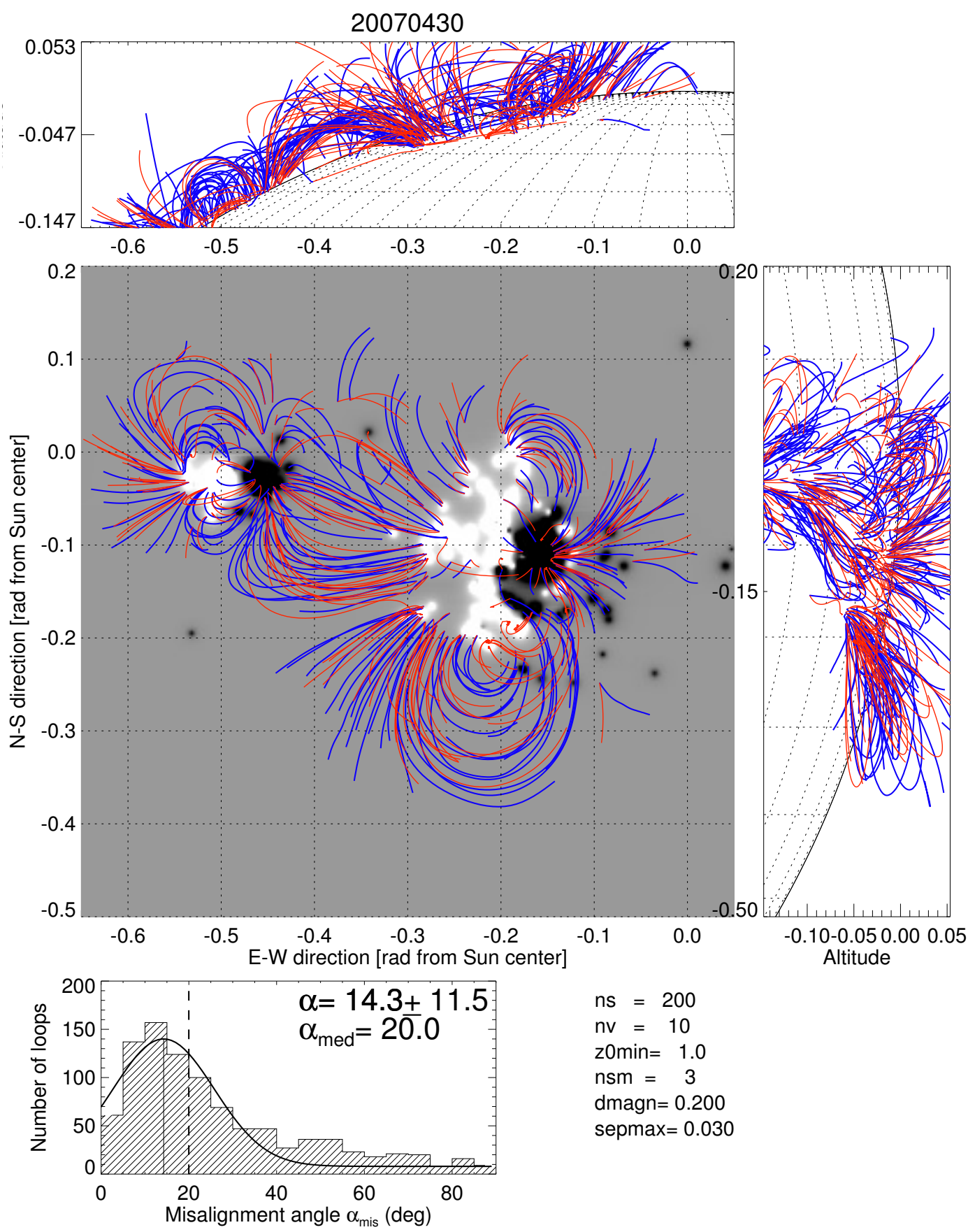

Figure 30: Best-fit potential field model of AR observed on 2007 Apr 30. The stereoscopically triangulated loops are shown in blue color, while field lines starting at identical footpoints as the STEREO loop extrapolated with the best-fit potential field (composed of $n_{c}=200$ unipolar magnetic charges) are shown in red. Side views are shown in the top and right panels. A histogram of misalignment angles measured between the two sets of field lines is displayed in the bottom panel. The distribution is fitted with a Gaussian, where the vertical solid line indicates the peak of the Gaussian (or most probable value), while the vertical dashed line indicates the median value (from Aschwanden and Sandman, 2010). 


\subsection{MHD oscillations in coronal loops}

A review of the $3 \mathrm{D}$ geometry, motion, and hydrodynamic aspects of oscillating coronal loops is given in Aschwanden (2009c), which describes two types of 3D reconstructions of kink-mode loop oscillations: (i) Deprojection of 2D loop tracings using the method of curvature radius maximization, and (ii) stereoscopic triangulation of loop tracings, both applied as a function of time. The time-dependent 3D reconstruction of oscillating loops, $[x(t), y(t), z(t)]$, yields vital diagnostic on the standing (eigen-modes) and/or propagating MHD waves, and on the linear and/or circular polarization of the excited wave modes. We describe here only the stereoscopic results.

In a survey of flare and CME events observed with the SECCHI/EUVI telescope during the first two years of the mission, at least 10 events were identified that contain loop oscillations and/or propagating waves (Aschwanden et al., 2009b), which we list in Table 2 (Movies and quicklook plots of these oscillation events are also available at http://secchi.lmsal.com/EUVI/). This list of events may not be complete, because oscillating loops and propagating waves were mostly identified by visual inspection of EUVI $171 \AA$ movies (either by some periodic motion over at least two periods or by some global wave propagation), rather than by automated detection algorithms that scan the entire EUVI database. Inspecting the spacecraft separation angle in Table 2 we see that 9 events have been observed at separation angles of $\alpha_{\text {sep }} \approx 6^{\circ}-47^{\circ}$ and are thus suitable for stereoscopic $3 \mathrm{D}$ reconstruction.

One loop oscillation event occurred during the 2007 Jun 27, 17:30 UT, flare and has been analyzed in several studies (Verwichte et al., 2009; Aschwanden et al., 2009b; Aschwanden, 2009c). No stereoscopic 3D reconstruction with a triangulation method has been attempted in the first study on this loop, because the loop footpoints are not visible in one spacecraft (STEREO/A) and cannot easily be located in the image of the other spacecraft (STEREO/B), as well as corresponding features of the upper loop segment are difficult to verify in both images (Verwichte et al., 2009). Instead, a semi-circular loop geometry was fitted in projection to both stereoscopic images by varying the inclination angle of the loop plane, leading to a loop radius of $r_{\text {loop }}=110 \mathrm{Mm}$, an azimuth angle (of the loop baseline) of $\alpha=29^{\circ}$ (anti-clockwise from the heliographic east-west direction), and an inclination angle of $\theta=27^{\circ}$. The $2 \mathrm{D}$ projection of this $3 \mathrm{D}$ geometry in the LOS directions of STEREO/A and B is shown in Figure 31. The time behavior of the oscillating loop is shown in a sequence of running-difference images in Figure 32, which displays clear kink-mode displacements (black/white loop rims) for the considered loop. A time-slice plot obtained along a cross-sectional slice reveals the damped sinusoidal oscillation (Figure 33), whose amplitude could be fitted by

$$
a(t)=a_{0}+a_{1} \cos \left(\frac{2 \pi\left(t-t_{0}\right)}{P}+\Phi\right) \exp \left(-\frac{t-t_{0}}{\tau_{d}}\right)
$$

yielding an initial amplitude of $a_{1}=2.5$ EUVI pixels $(2900 \mathrm{~km})$, a period of $P=565 \mathrm{~s}(\approx 9 \min )$, and an exponential damping time of $\tau_{d}=1600 \mathrm{~s}(\approx 27 \mathrm{~min})$, similar to the values found by Verwichte et al. (2009). Note that the EUVI cadence is $150 \mathrm{~s}(2.5 \mathrm{~min})$.

In a subsequent study (Aschwanden, 2009c), stereoscopic triangulation has been applied to the oscillating loop and the reconstructed 3D geometry is shown in Figure 34: The XY-projection in the image plane of STEREO/A and B is rendered in the left-hand panels, and the YZ-projections as viewed from the east direction at the east limb are shown in the right-hand panels. The spacecraft separation angle at that time was $\alpha_{\text {sep }}=8.26^{\circ}$. The triangulation has been repeated three times with independent manual tracings and the three solutions are shown in Figure 35, projected parallel and perpendicular to the average loop plane. Surprisingly, the triangulated loop reveals an S-shaped geometry in the vertical projection top-down (Figure 35, right panels), and a heavily deformed asymmetry in the side-on projection (Figure 35, middle panels). Thus, the oscillating loop seems to be neither circular nor coplanar. This is an intriguing new result that could not have been obtained without STEREO observations.

Living Reviews in Solar Physics

http://www. livingreviews.org/lrsp-2011-5 
Table 2: Flare and CME events with loop oscillations and waves observed with STEREO/EUVI (Aschwanden, 2009c).

\begin{tabular}{|c|c|c|c|c|c|c|c|c|c|c|c|}
\hline \multirow[t]{2}{*}{$\#$} & \multirow[t]{2}{*}{ Date } & \multirow{2}{*}{$\begin{array}{l}\text { Time } \\
(\mathrm{UT})\end{array}$} & \multirow{2}{*}{$\begin{array}{l}\text { Heliogr. } \\
\text { location }\end{array}$} & \multirow{2}{*}{$\begin{array}{c}\text { Stereo } \\
\text { angle } \\
\text { (deg) }\end{array}$} & \multirow{2}{*}{$\begin{array}{c}\text { GOES } \\
\text { class }\end{array}$} & \multicolumn{2}{|c|}{ RHESSI $^{1}$} & \multicolumn{3}{|c|}{$\mathrm{EUVI}^{2}$} & \multirow{2}{*}{$\begin{array}{c}\mathrm{CME}^{3} \\
\text { Rep. }\end{array}$} \\
\hline & & & & & & $\begin{array}{c}\mathrm{E} \\
(\mathrm{keV})\end{array}$ & $\begin{array}{c}\mathrm{P} \\
(\mathrm{cts} / \mathrm{s})\end{array}$ & $\begin{array}{c}\text { A } \\
\left(10^{5}\right.\end{array}$ & $\begin{array}{c}\mathrm{B} \\
\mathrm{DN} / \mathrm{s})\end{array}$ & Com. & \\
\hline 8 & $07 / 01 / 12$ & $01: 00-03: 00$ & N00W87 & 0.2 & $\mathrm{C} 1.5$ & 12 & 34 & 31.2 & & IPEW & $\mathrm{L}$ \\
\hline 35 & $07 / 05 / 02$ & $23: 20-23: 59$ & S09W16 & 6.3 & $\mathrm{C} 8.5$ & 12 & 30 & 23.0 & 17.3 & IPW & $\ldots$ \\
\hline 43 & $07 / 05 / 16$ & $17: 10-18: 10$ & N03E34 & 8.2 & $\mathrm{C} 2.9$ & 12 & 26 & 12.7 & 9.9 & IPDW & LS \\
\hline 46 & $07 / 05 / 19$ & $12: 40-13: 20$ & N03W03 & 8.6 & B1.3 & 12 & 46 & 17.4 & 13.9 & IPEDW & LS \\
\hline 100 & $07 / 06 / 08$ & $12: 30-13: 30$ & S08W08 & 11.9 & B7.6 & 12 & 28 & 7.9 & 5.9 & IPDW & LS \\
\hline 104 & $07 / 06 / 09$ & $13: 20-14: 40$ & S10W23 & 12.1 & M1.0 & 12 & 184 & 69.4 & 45.8 & IW & $\mathrm{L}$ \\
\hline 109 & $07 / 06 / 27$ & $17: 30-18: 30$ & S20E89 & 15.4 & C1.3 & 3 & 34 & 2.9 & 2.4 & IDOW & $\ldots$ \\
\hline 133 & 07/08/06 & $15: 20-15: 50$ & S12E38 & 23.3 & C1.1 & 12 & 32 & 7.8 & 25.2 & IPW & LS \\
\hline 175 & $07 / 12 / 31$ & $00: 30-01: 40$ & S15E87 & 44.0 & $\mathrm{C} 8.3$ & 25 & 288 & 7.5 & 39.3 & IPDOW & LS \\
\hline 183 & $08 / 03 / 25$ & $18: 30-19: 30$ & S25E90 & 47.2 & M1.7 & 12 & 944 & 17.8 & 192.2 & IPDEW & LS \\
\hline
\end{tabular}

\#) Event identification number in EUVI flare and CME catalog:

http://secchi.lmsal.com/EUVI/euvi_events.txt

1) RHESSI:

$\mathrm{E}=$ highest detected energy range: $3-6,6-12,12-25,25-50,50-100 \mathrm{keV}$, $\mathrm{P}=$ peak count rate of RHESSI light curve.

$\left.{ }^{2}\right)$ EUVI comments:

$\mathrm{A}=$ background-subtracted peak flux detected in EUVI/A,

$\mathrm{B}=$ background-subtracted peak flux detected in EUVI/B,

$\mathrm{D}=$ Dimming in EUV,

$\mathrm{E}=$ Eruptive feature,

$\mathrm{I}=$ Impulsive EUV emission (simultaneous with hard X-rays),

$\mathrm{O}=$ Occulted (for A if flare position is east, or for B if west),

$\mathrm{P}=$ Postflare loop emission,

$\mathrm{W}=$ Waves or oscillations.

3) CME reports:

$\mathrm{L}=\mathrm{LASCO} / \mathrm{SOHO}$,

$\mathrm{S}=\mathrm{SECCHI}$ Cor-1 or Cor-2.

If we fit a circular geometry to the same 2D loop tracings (dashed yellow curves in Figure 34), we obtain from the average and standard deviations of 3 independent tracings the following parameters: a baseline azimuth angle of $\alpha=27.0^{\circ} \pm 4.5^{\circ}$, a loop plane inclination angle of $\theta=24.1^{\circ} \pm 1.0^{\circ}$, a loop radius of $r=83 \pm 10 \mathrm{Mm}$, and a full loop length of $L=319 \pm 19 \mathrm{Mm}$. The full 3D loop length of the triangulated loop is slightly shorter than the circular model, i.e., $L=311 \pm 22 \mathrm{Mm}$. This compares favorably with the semi-circular forward-fitting of Verwichte et al. (2009), who obtained values of $\alpha=19^{\circ}, \theta=27^{\circ}, r=110 \mathrm{Mm}$, and $L=346 \mathrm{Mm}$. Thus, we conclude that the circular forward-fitting model yields values that are very close to those of the stereoscopic method, differing by $\lesssim 10^{\circ}$ for the loop plane inclination angle and for the loop length, which is the most important parameter for coronal seismology. However, the forward-fitting model makes the assumption of circularity and coplanarity and, thus, cannot reveal asymmetric and non-planar shapes of the oscillating loop.

In Figure 35, we transformed the loop coordinates from the image coordinate system $\left(x_{A}, x_{B}, x_{Z}\right)$ into the loop coordinate system $(X, Y, Z)$, which is defined in the following way: the horizontal $X$-axis is aligned with the loop baseline between the two loop footpoints, with the midpoint as center $X=0$. The vertical $Z$-axis intersects the baseline midpoint and the apex midpoint of the loop, while the $Y$-axis is perpendicular to the $X-Z$ plane. The coordinate transformation is ac- 

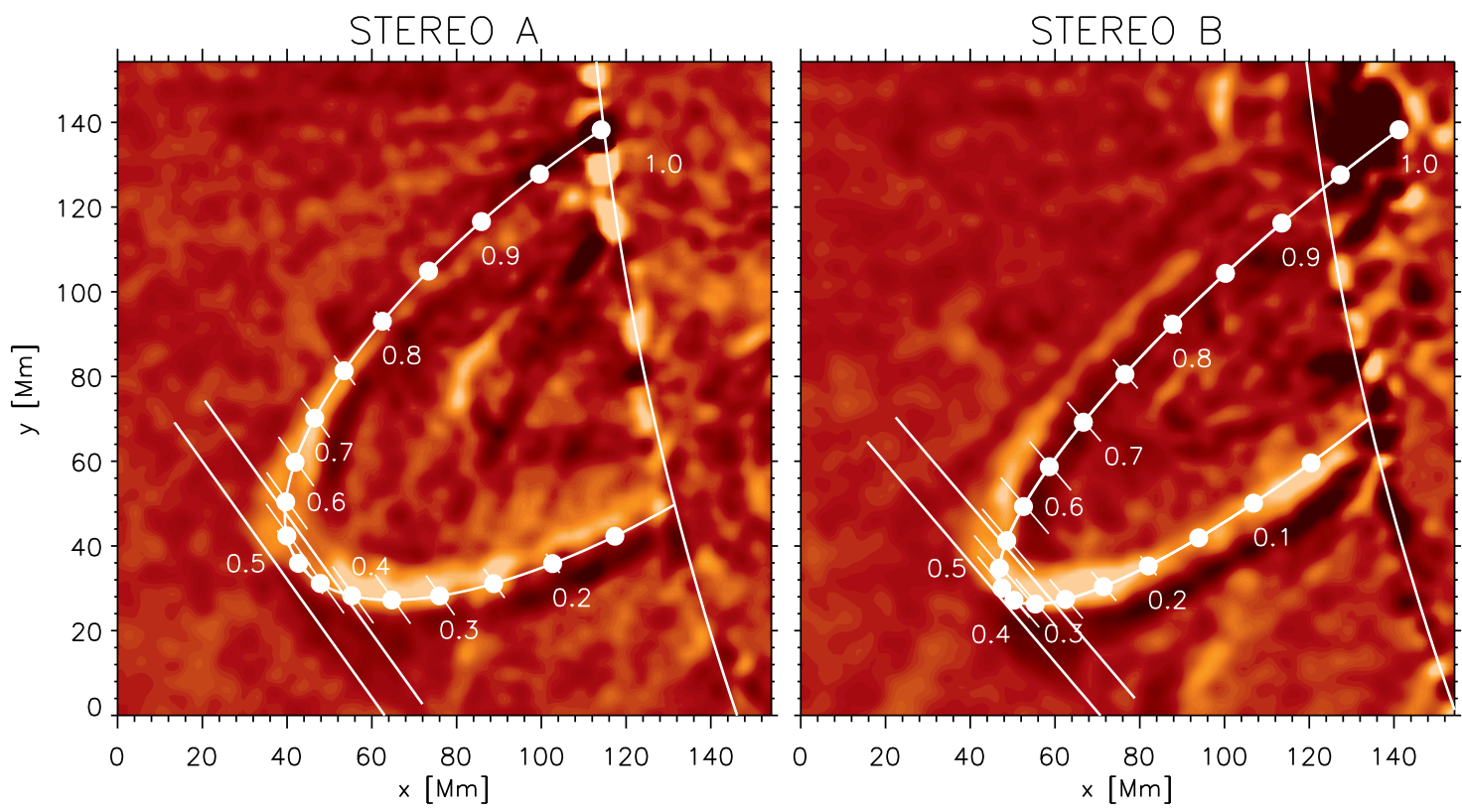

Figure 31: 3D reconstruction of an oscillating loop observed during the flare of 2007 Jun $27, \approx 18: 19$ UT, superimposed on the STEREO/A (left) and STEREO/B images (right), obtained by stereoscopic fitting of a circular loop geometry (from Verwichte et al., 2009).

complished by 3 subsequent rotations (i.e., by the azimuth angle $\alpha$, the loop plane inclination angle $\theta$, and the longitude angle $l_{1}$, see Figure 23). The oscillating loop shown in Figure 35 is clearly heavily deformed and deviates significantly from a coplanar and circular model. The maximum deviations $\Delta r$ from the average loop radius $r$ are $0.54<(\Delta r / r)<1.45$, and the non-planarity reaches up to $\lesssim 0.21$ of the loop radius, which is much larger than for typical active region loops, which have radial variations of $\lesssim 1.2$ and non-planarity deviations of $\lesssim 0.1$. Obviously, this loop is highly non-circular and non-planar, possibly affected by the dynamic magnetic forces associated with the launch of a CME that usually is responsible for triggering loop oscillations.

Using the method of stereoscopic 3D triangulation, as shown for a single time frame at 2007 Jun 27, 18:18:30 UT in Figure 34, we repeat now the same procedure for 12 (epipolar-coaligned) stereoscopic image pairs in the time interval of 17:58-18:26 UT. The same time sequence is shown in form of running-time difference images in Figure 32, yielding an oscillation period of $P=$ $565 \mathrm{~s}$ and an exponential damping time of $\tau_{\text {damp }} \approx 1600 \mathrm{~s}$. The triangulated $3 \mathrm{D}$ geometry of the oscillating loop is shown in Figure 36, which has a consistent asymmetric S-shape throughout the entire observed time interval $(\approx 35 \mathrm{~min})$ as shown in Figure 34 , while the oscillation amplitude is much smaller than the deviations of the loop geometry from a circular model. We display the average amplitude of loop motions in $x$-direction (east-west amplitude $d x(t)$ in Figure 36 top right panel), and in $z$-direction (line-of-sight amplitude $d z(t)$ in Figure 36 middle right panel). Both amplitudes show a correlated oscillation with similar periods and amplitudes, i.e., $P=681 \mathrm{~s}$ for $d x(t)$ and $P=685 \mathrm{~s}$ for $d z(t)$, and $A_{0}=1.4 \mathrm{Mm}$ for $d x(t)$ and $A_{0}=1.5 \mathrm{Mm}$ for $d z(t)$, respectively. The amplitude $d x(t)$ corresponds to an oscillation in the vertical plane (with respect to the solar surface), and $d z(t)$ to an oscillation in the horizontal plane, but the two oscillations are almost in phase $\left(t_{0} / P=0.19\right.$ vs. $\left.t_{0} / P=0.26\right)$. The fact that the two oscillation directions have a similar amplitude implies that the polarization is a combination of horizontal and vertical planes

Living Reviews in Solar Physics

http://www . livingreviews . org/lrsp-2011-5 

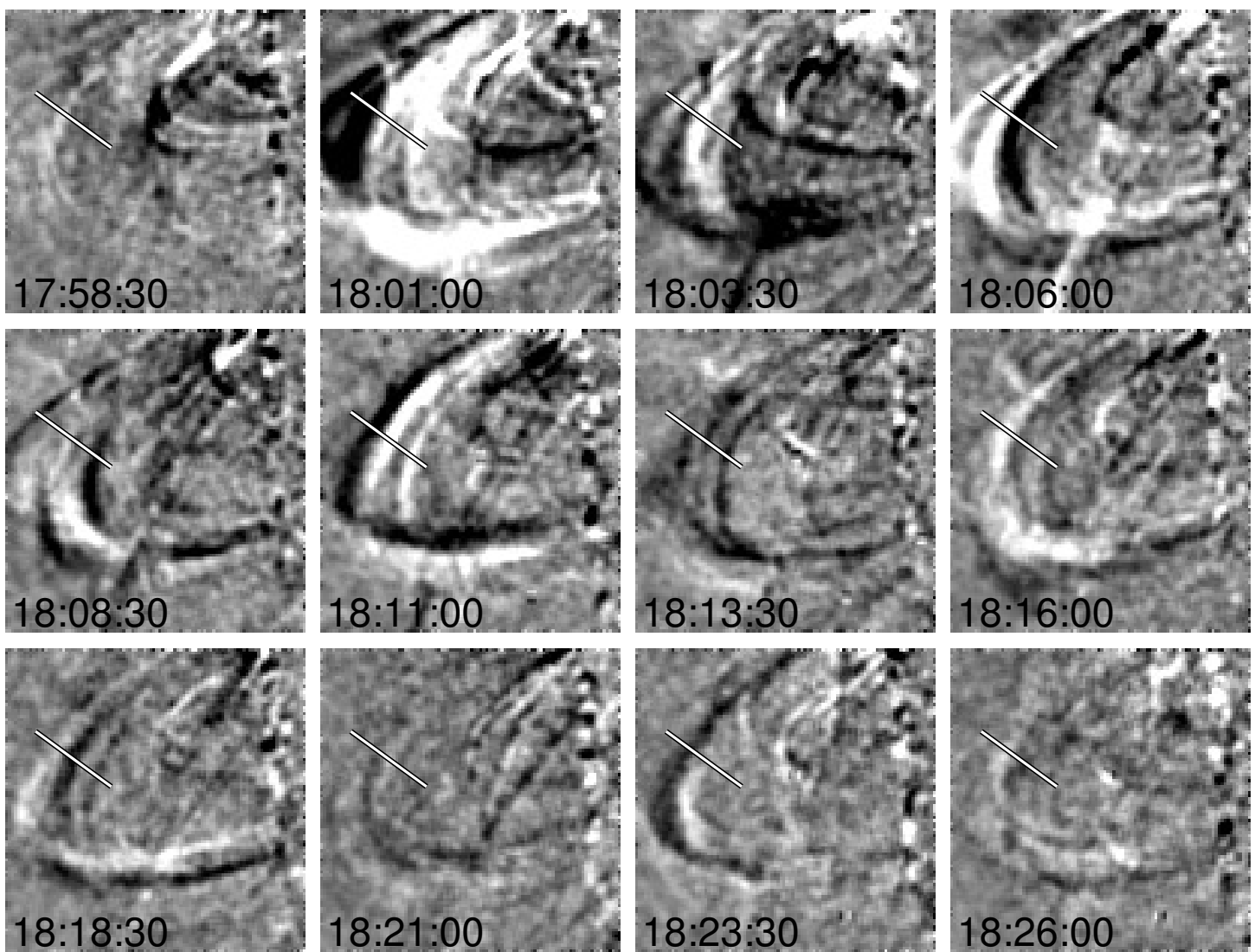

Figure 32: Sequence of running-difference EUVI/B images in the area of the oscillating loop during the time period of 2007 Jun 26, 17:56:00 UT and 18:26:00 UT. The amplitude measurement of the oscillating loop is carried out along the cross-sectional slit marked with a diagonal bar (from Aschwanden et al., 2009b). 

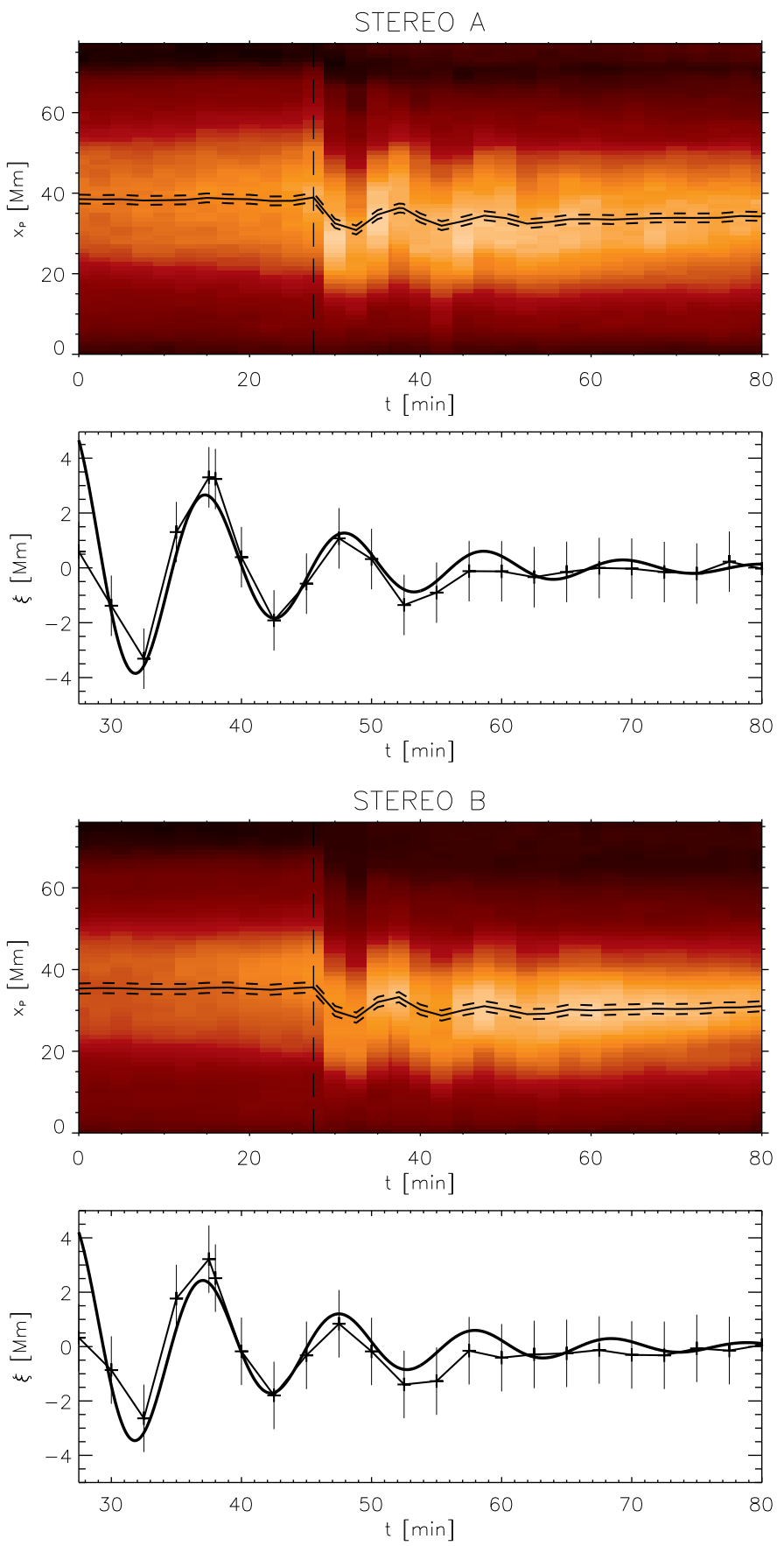

Figure 33: Time-slice plots (color) and amplitude of kink mode as a function of time with fitted damped sine function (graphs) for both STEREO/A and B spacecraft, for the loop oscillation event of 2007 Jun 27, 17:30 UT (from Verwichte et al., 2009). 

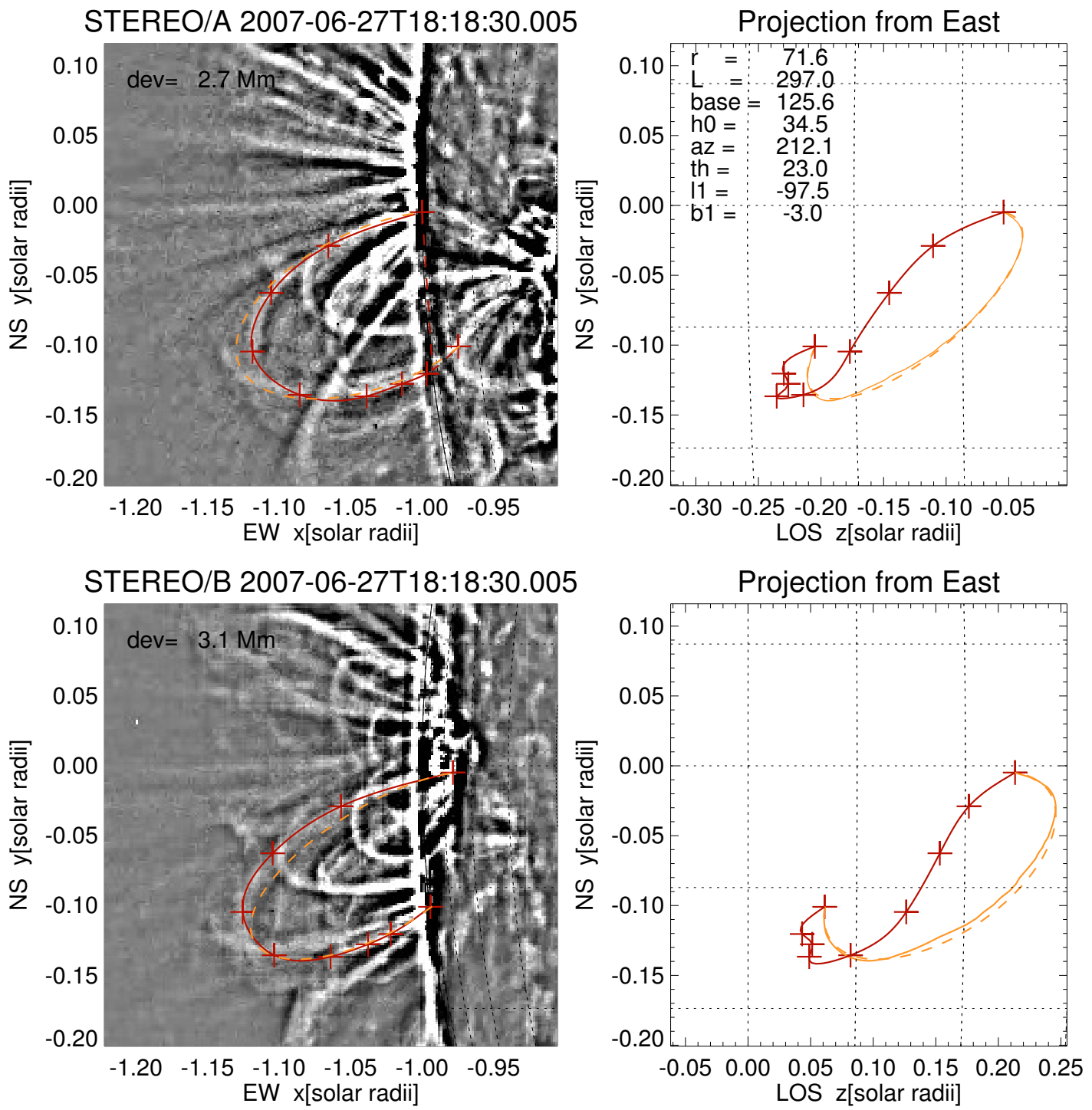

Figure 34: Still from a movie showing 3D reconstruction of the same oscillating loop as Figure 32, observed on 2007 Jun 27, 18:19 UT, superimposed on a highpass-filtered image of STEREO/A (bottom) and STEREO/B image (top), using the stereoscopic triangulation method. The loop shape is traced with 9 points (red crosses), interpolated with a $2 \mathrm{D}$ spline (red curve), and fitted with an elliptical geometry (yellow curve). The circular model (yellow curve) and the solution of the 3D reconstruction (red curve) is also projected into the $(z, y)$ plane (right panels), with $z$ being the LOS (from Aschwanden, 2009c). (To watch the movie, please go to the online version of this review article at http://www.livingreviews.org/ lrsp-2011-5.) 

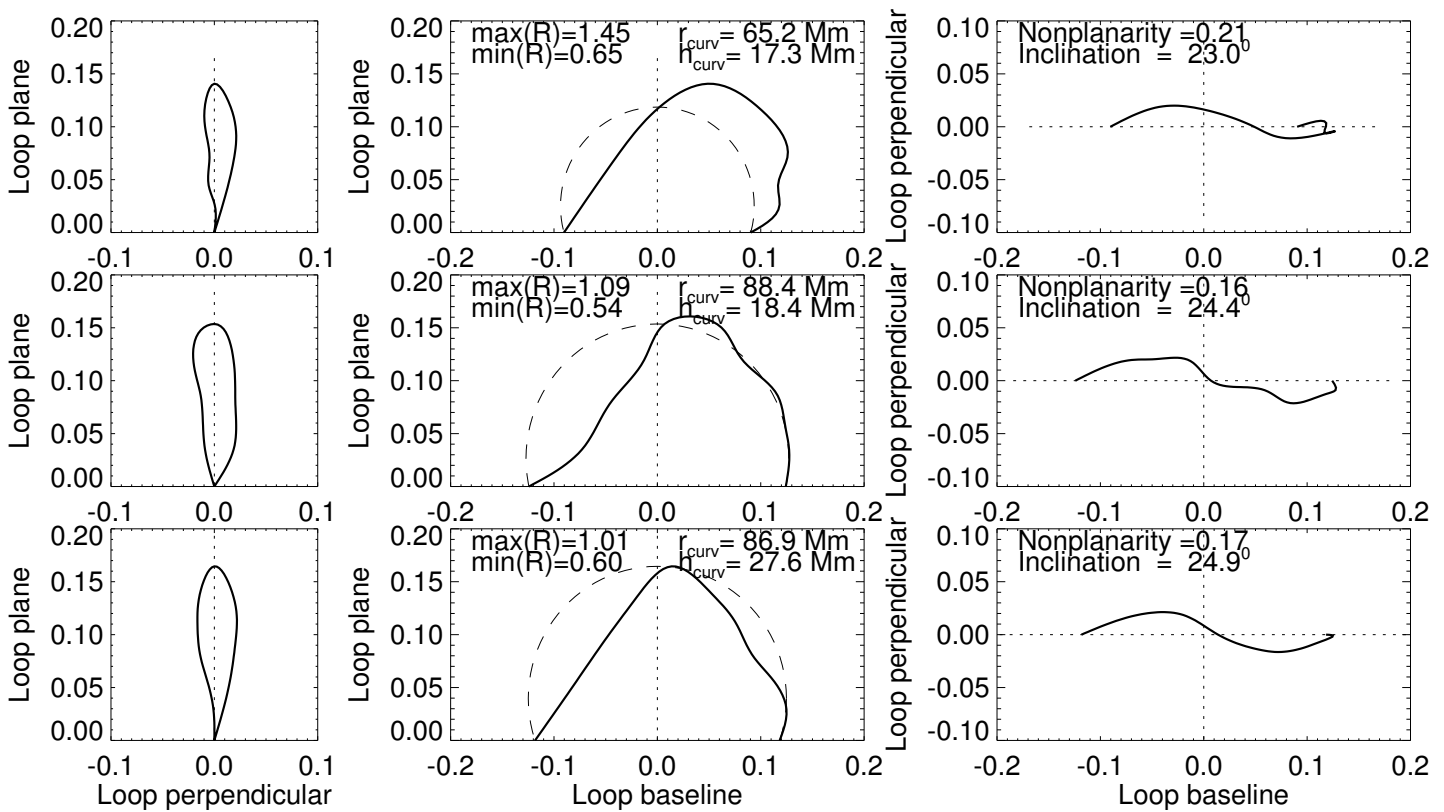

Figure 35: Projections of the reconstructed 3D loop geometry (see Figure 34) into the 3 orthogonal planes of the loop coordinate system: edge-on (left), side-on (middle), and top-down (right). The three rows present 3 independent trials of manual loop tracings.

with a polarization angle near $45^{\circ}$. However, since the average 3D loop geometry corresponds to a non-planar, helically-twisted shape, the 3D motion can also be described in terms of circular polarization or a torsional mode. It is instructive to inspect a movie visualization of Figure 36.

In Figure 37 we show the triangulated loop motion projected into the 3 orthogonal directions of the loop coordinate system. In order to illustrate the plausibility of circular polarized kink motion we simulate a helically twisted loop (with a twist of 0.75 turns and geometric tapering of the toroidal radius towards the footpoints) that undergoes a periodical change in the twist angle, where the displacement is highest at the loop apex and falls off towards the footpoints. This simple simulation shown in Figure 37 (bottom half) demonstrates qualitatively how the observed loop shapes and motions (Figure 37 top half) can be explained by a circular polarization. Some further modeling studies are required to decide whether we deal with a circularly polarized (helical) kink mode or with a torsional wave mode. Torsional wave modes and helical loop geometries are of particular interest for triggering mechanisms of flares and CMEs by the kink instability. A large twist angle of $\Phi=12 \pi$ was recently observed to trigger a flare (Srivastava et al., 2010). In addition, numerical MHD simulations with realistic initial conditions for the excitation mechanisms may shed some light on the coupling of various known MHD wave modes, i.e., kink-modes, sausage-modes, and torsional modes (Ofman, 2009).

\subsection{MHD waves in coronal loops}

Besides fast-mode MHD waves, which can be subdivided into kink-mode (asymmetric, see Section 4.5) and sausage-mode (symmetric) types, there are also slow-mode MHD waves, which propagate with acoustic (sound) speed. The sound speed can be expressed in terms of the electron temperature $T_{e}$ (assuming the coronal approximation $T_{e}=T_{i}$, although $T_{i}>T_{e}$ according to some 

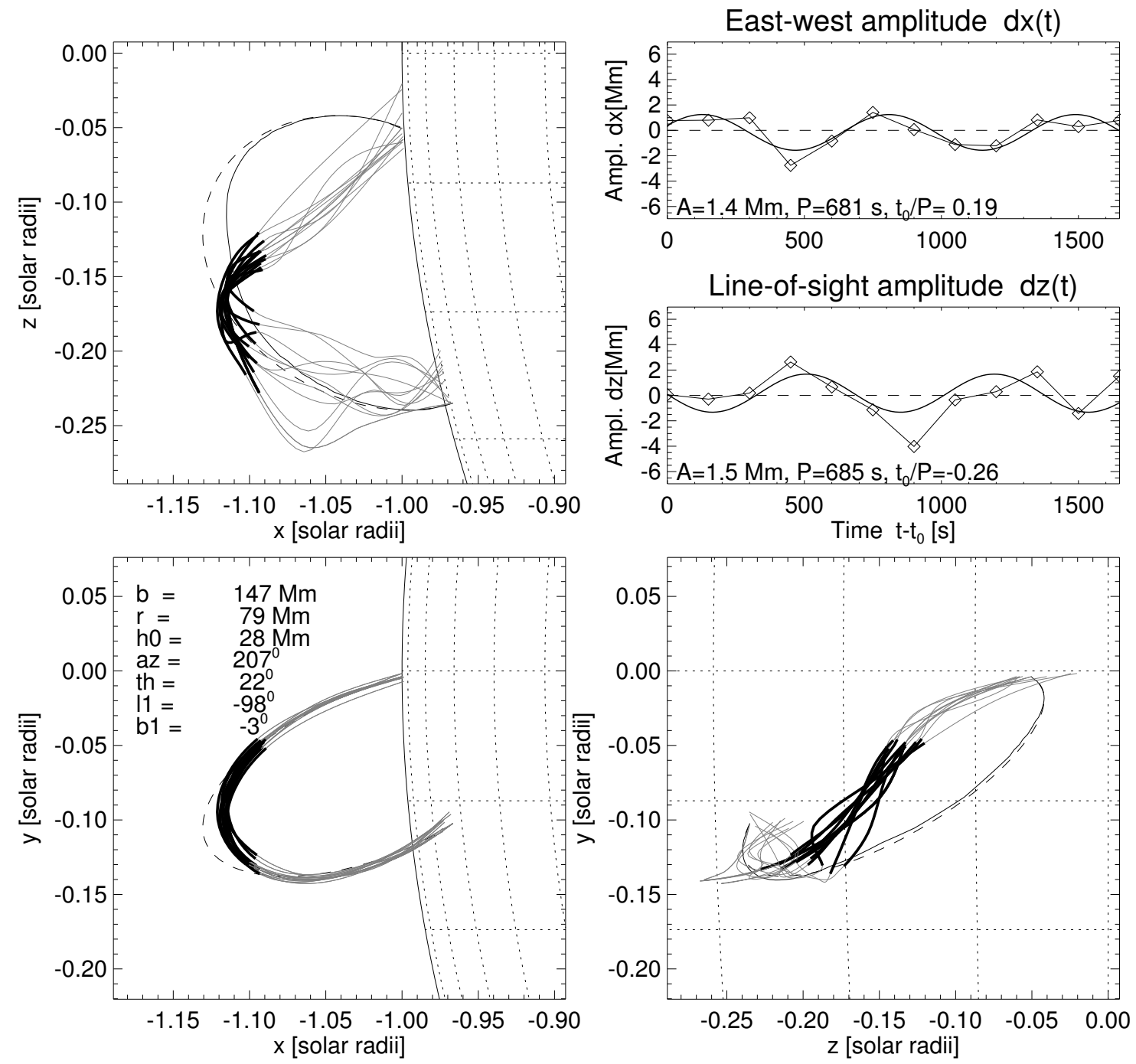

Figure 36: Still from a movie showing 3D reconstruction of loop oscillations for a sequence of 16 EUVI/A+B $171 \AA$ images in the time interval of 2007 Jun 27, 17:58-18:26 UT, using the stereoscopic triangulation method. The loop tracings in EUVI/A are rendered in the $x-y$ plane (bottom left panel), while the orthogonal reconstruction are shown in the $x-z$ plane (top left panel) and in the $z$-y plane (bottom right panel). The loop tracings are rendered with grey curves, the semi-circular fit with a dashed curve, and the curvature radius maximization method with a thin black curve. The oscillation amplitudes averaged in the loop segments $0.3<s / L<0.6$ (marked with thick black curves) are shown in $x$-direction (east-west amplitude $d x(t)$ in top right panel) and in the $z$ direction (line-of-sight amplitude $d z(t)$ in middle right panel). (To watch the movie, please go to the online version of this review article at http://www.livingreviews.org/lrsp-2011-5.) 

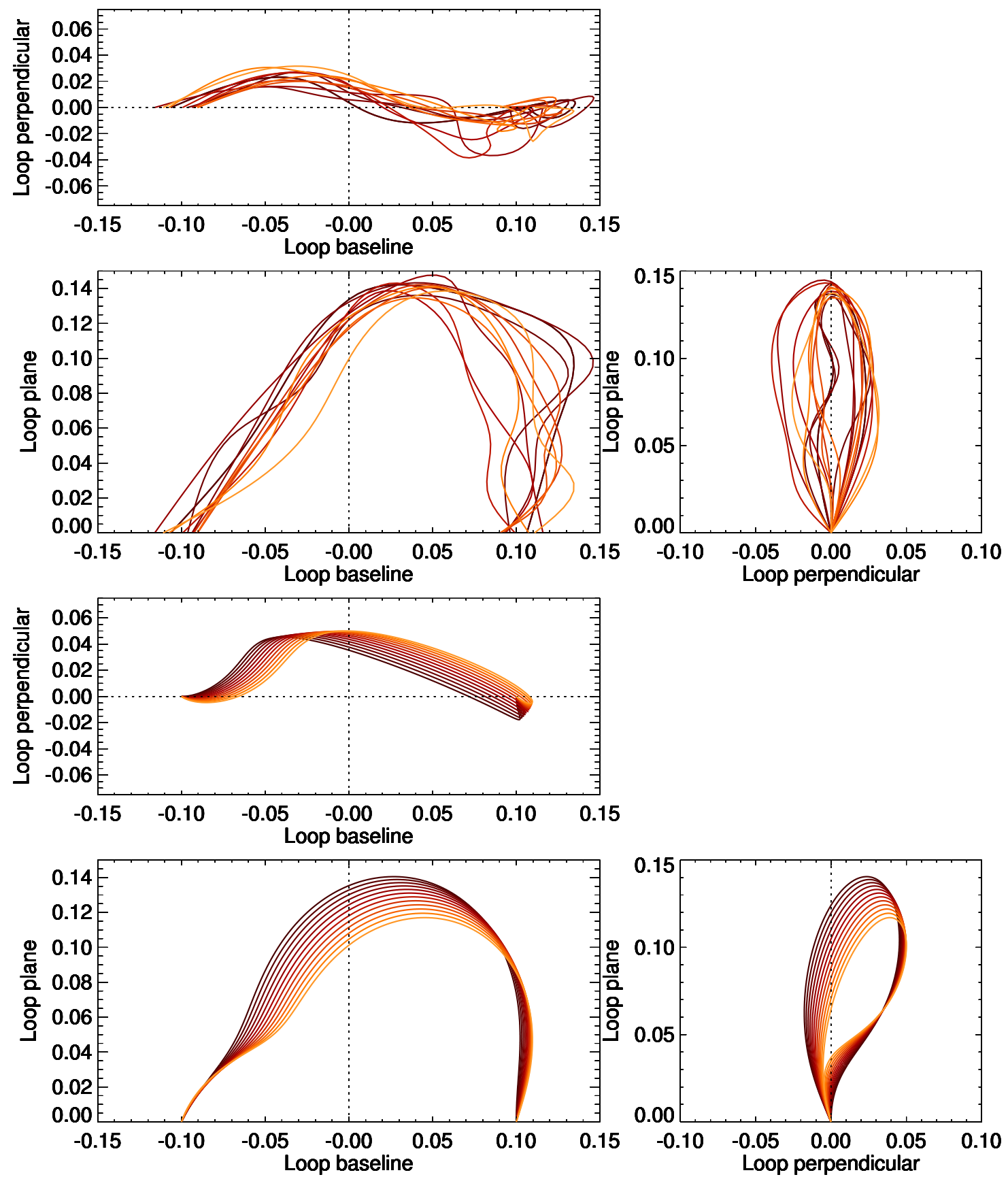

Figure 37: Still from a movie showing Orthogonal projections of a triangulated oscillating loop (frames in top half) during the same time interval as shown in Figure 13 (with the time marked by colors, progressing in order of brown-red-orange-yellow). The bottom panels visualize the same projections of a helically twisted model loop (see model parameters in text). (To watch the movie, please go to the online version of this review article at http://www.livingreviews.org/lrsp-2011-5.) 
observations),

$$
c_{s}=\left(\frac{\gamma p_{t h}}{\rho}\right)^{1 / 2}=1.66 \times 10^{4} \sqrt{\frac{T_{e}}{\mu}} \quad\left[c \mathrm{cs}^{-1}\right],
$$

where $\gamma=5 / 3$ is the adiabatic index, $p_{t h}=2 n_{e} k_{B} T_{e}$ the thermal pressure, $\rho=n_{i} \mu m_{p}$ the mass density, and $\mu=1.27$ the mean molecular weight. However, if a sound wave propagates at an angle of $\theta$ to the line-of-sight, the observed speed scales with the sine function of the line-of-sight angle, i.e., $v_{\mathrm{obs}}=c_{s} \sin \phi$. This projection angle $\phi$ can be determined by stereoscopic triangulation of the loop structure that guides the sound wave propagating in longitudinal direction.

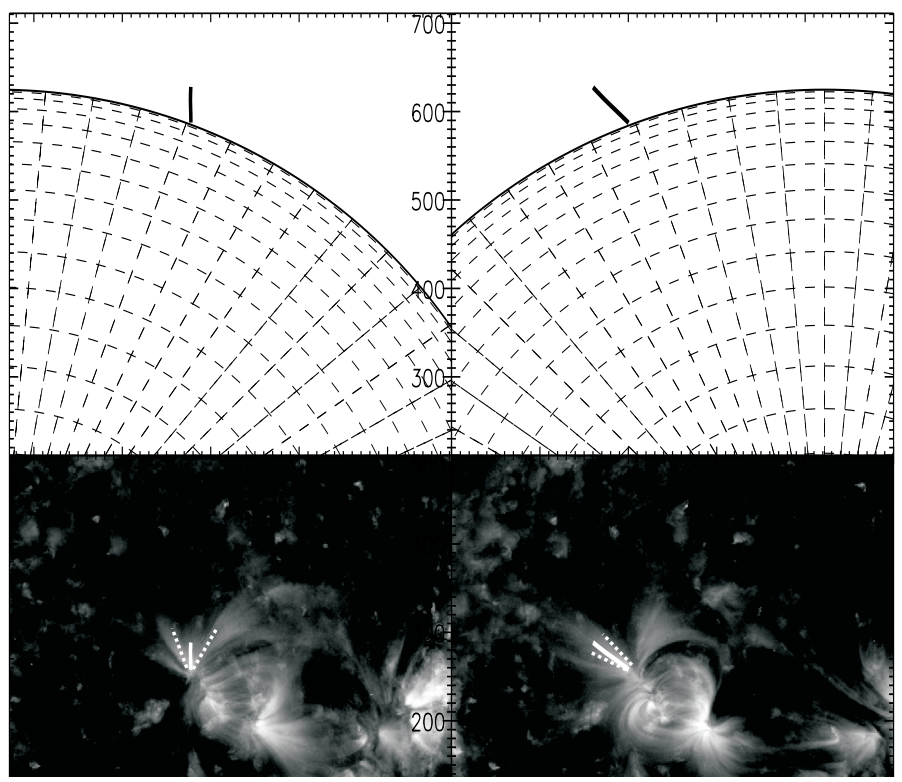

Figure 38: Derived 3D geometry of the wave propagation along the coronal (fan) loop, observed on 2008 Jan 10 with STEREO/A and B (bottom panels) The top panels show the projected geometry of the propagation vector with the view rotated by $90^{\circ}$ seen from solar north (from Marsh et al., 2009).

Such a measurement has been conducted for slow-mode (acoustic) waves observed with STEREO/A and B on 2008 Jan 10 (Marsh et al., 2009). The propagating waves were observed in a fan-like loop structure emanating from one magnetic pole of a bipolar active region (Figure 38). A time-slice plot of the EUV flux along the fans as a function of time is shown in Figure 39, which confirms the quasi-periodic wave propagation (diagonal pattern), for which a velocity of $v_{A}=62.9 \pm 0.1 \mathrm{~km} \mathrm{~s}^{-1}$ was measured with STEREO/A, and $v_{B}=104.1 \pm 0.2 \mathrm{~km} \mathrm{~s}^{-1}$ with STEREO/B, respectively. The wave period was found to be $P \approx 12 \mathrm{~min}$. Stereoscopic triangulation of the fans yielded an inclination angle of $\theta=37^{\circ} \pm 6^{\circ}$ to the local vertical. 3D projections of the triangulated directions are also shown in Figure 38 (top panels). Correcting the observed wave speed for the corresponding line-of-sight angle yields a corrected speed of $c_{A}=132 \pm 9 \mathrm{~km} \mathrm{~s}^{-1}$ and $c_{B}=132 \pm 11 \mathrm{~km} \mathrm{~s}^{-1}$, which represents the first measurement of the true coronal longitudinal slow-mode speed $c_{s}$ in $3 \mathrm{D}$. Inserting this true sound speed into Eq. (43), a temperature of $T_{e}=0.84 \pm 0.15 \mathrm{MK}$ is inferred, which is close to the peak sensitivity of the used $171 \AA$ passband. The temperature structure of the same fan loop was also investigated with EIS/Hinode and a mean temperature of $T_{e}=0.89 \pm 0.09 \mathrm{MK}$ was obtained, in excellent agreement with the EUVI measurements, which confirms that the slow-mode phase speed is identical to the sound speed (Marsh and Walsh, 2009). 

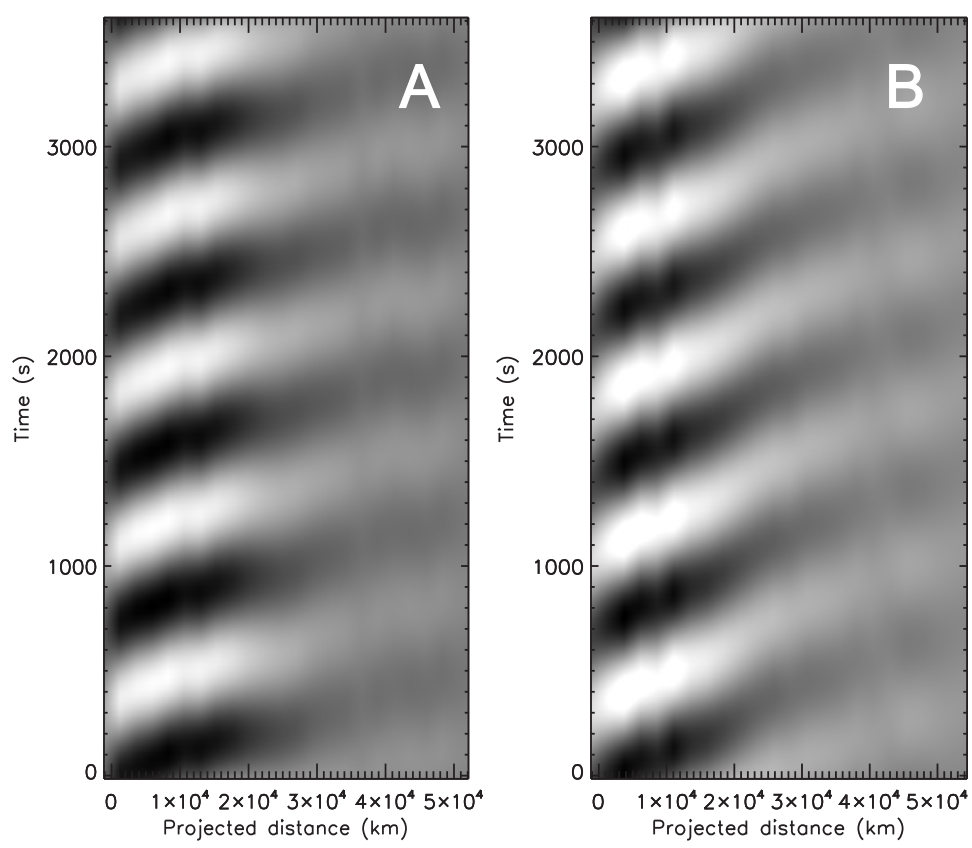

Figure 39: Time-slice plots of the integrated intensity along the (fan) loops for STEREO/B (left) and A (right). The abscissa indicates the observed distance long the loop, perpendicular to the line-of-sight from each spacecraft (from Marsh et al., 2009).

\subsection{Quiescent filaments and prominences}

EUV filaments (when seen in absorption on the solar disk) or prominences (when seen in emission above the limb) reveal the location of the magnetic neutral line in active regions and often represent progenitors of CMEs. They may exist in a quiescent state for a long time, but some become magnetically unstable and initiate the eruptive state of a CME. Analysis of spectra and images of prominence plasma is not trivial, since non-LTE (departure from Local Thermodynamic Equilibrium) radiative transfer models are required (see recent review by Labrosse et al., 2010b).

Some attempts of 3D reconstruction of quiescent EUV filaments have been made in the preSTEREO era, using the solar rotation and SOHO/EIT data (Foullon, 2003). The 3D geometry of filaments can often be approximated by a vertical slab or sheet-like plasma structure. A method to estimate the width and inclination of such filament sheets has been applied to STEREO He II $304 \AA$ data (Gosain and Schmieder, 2010). On the other side, helical twisted flux ropes have also been observed as precursors of filaments (Raouafi, 2009). The automated detection of filaments in He $304 \AA$ images is often difficult due to the varying brightness level of the background, but useful methods have been developed to remove this background (Artzner et al., 2010). Automated detection of limb prominences in He II $304 \AA$ data has been developed, which can discriminate limb prominences from active regions or the quiet corona in $93 \%$ of the cases (Labrosse et al., 2010a). Another solar prominence catcher and tracker (SLIPCAT) algorithm produced a catalog of 9477 well-tracked prominences during the 2007 April-2009 October period of STEREO $304 \AA$ observations (Wang et al., 2010). The statistical results showed that most prominences occur below a latitude of $60^{\circ}$, have a length of $\approx 50 \mathrm{Mm}$, a height of $\approx 26 \mathrm{Mm}$, and $80 \%$ show no obvious motion (Wang et al., 2010). Another statistical study of 68 quiescent filament channels with STEREO/EUVI and Hinode/XRT revealed an asymmetry in the morphology due to the variation in axial flux of the flux rope along the channel (Su et al., 2010).

Living Reviews in Solar Physics

http://www . livingreviews . org/lrsp-2011-5 
A particular topology is the "prominence cavity", which is the limb manifestation of a longitudinally extended polar-crown filament channel (or tunnel), which has a lower density than the surrounding corona. The 3D geometry and morphology of such a prominence cavity has been reconstructed from STEREO data (Gibson et al., 2010).

A complex evolution of a quiescent and active filament, from the formation, interaction, to merging, accompanied by repeated heating via magnetic reconnection preceding the eruption on 2007 May 19, has been observed with STEREO (Bone et al., 2009). The same filament was stereoscopically triangulated and the height of the EUV emission was determined to $\approx 48 \pm 10 \mathrm{Mm}$ prior to eruption (Gissot et al., 2008; Liewer et al., 2009; Xu et al., 2010), which was found be higher than the location of $\mathrm{H} \alpha$ emission (Xu et al., 2010).

\subsection{Erupting filaments and prominences}

The best observed erupting filament during the STEREO era occurred on 2007 May 19 (Liewer et al., 2008, 2009; Culhane et al., 2008; Li et al., 2008; Gissot et al., 2008; Bone et al., 2009; Xu et al., 2010), early in the STEREO mission when the two spacecraft were separated by $8.5^{\circ}$, at an angle that is most suitable for stereoscopy. The filament could be followed in STEREO/EUVI $304 \AA$ from $\approx 12$ hours before to about 2 hours after the eruption, allowing to determine the 3D trajectory of the erupting filament (Figure 40), (Liewer et al., 2008, 2009). The trajectory of the erupting filament was also determined with an optical-flow (Velociraptor) algorithm (Gissot et al., 2008). The magnetic field topology was found to be highly non-potential, with a multipolar configuration, hosting frequent flares, multiple filament eruptions, and CMEs (Li et al., 2008). The 3D reconstruction of the filament and the chromospheric ribbons in the early stage of the eruption suggest that simultaneous heating occurred in the rising filament plasma and in the chromosphere below, as expected from a flare-like magnetic reconnection process (Liewer et al., 2009; Culhane et al., 2008). Simultaneous analysis of Hinode/SOT, TRACE, and EUVI data lead to conclusion that a pre-eruption sheared-core magnetic field is gradually destabilized by evolutionary tether-cutting flux cancellation, which was driven by converging photospheric flows, where the main filament ejection is triggered by flux cancellation between the positive flux elements and the surrounding negative field (Sterling et al., 2010). Comparisons of He II and $\mathrm{H} \alpha$ images show that emission in He II occurs together with disappearence in $\mathrm{H} \alpha$ and, thus, the disappearance results from heating and motion, rather than from draining and loss of filamentary material (Liewer et al., 2009).

STEREO observations of other eruptive filaments revealed also oscillating threads and filament disappearance (Gosain et al., 2009), a rotating ribbon-like 2D geometry rather than a twisted 3D flux tube (Bemporad, 2009; Bemporad et al., 2009), the eruption of a twisted filament (2008 Mar 25 ) that is consistent with the kink-unstable flux rope model or the sheared and opposite-polarity emerging twisted magnetic flux rope model (Aschwanden et al., 2009b), a slow gradual filament eruption with a weak but persistent acceleration of $3 \mathrm{~m} \mathrm{~s}^{-2}$ over 17 hours (Figure 41), followed by a gradual CME (Li et al., 2010a), and an initial mass off-loading phase that triggered the rise and catastrophic loss of equilibrium of a flux rope (Seaton et al., 2011). 


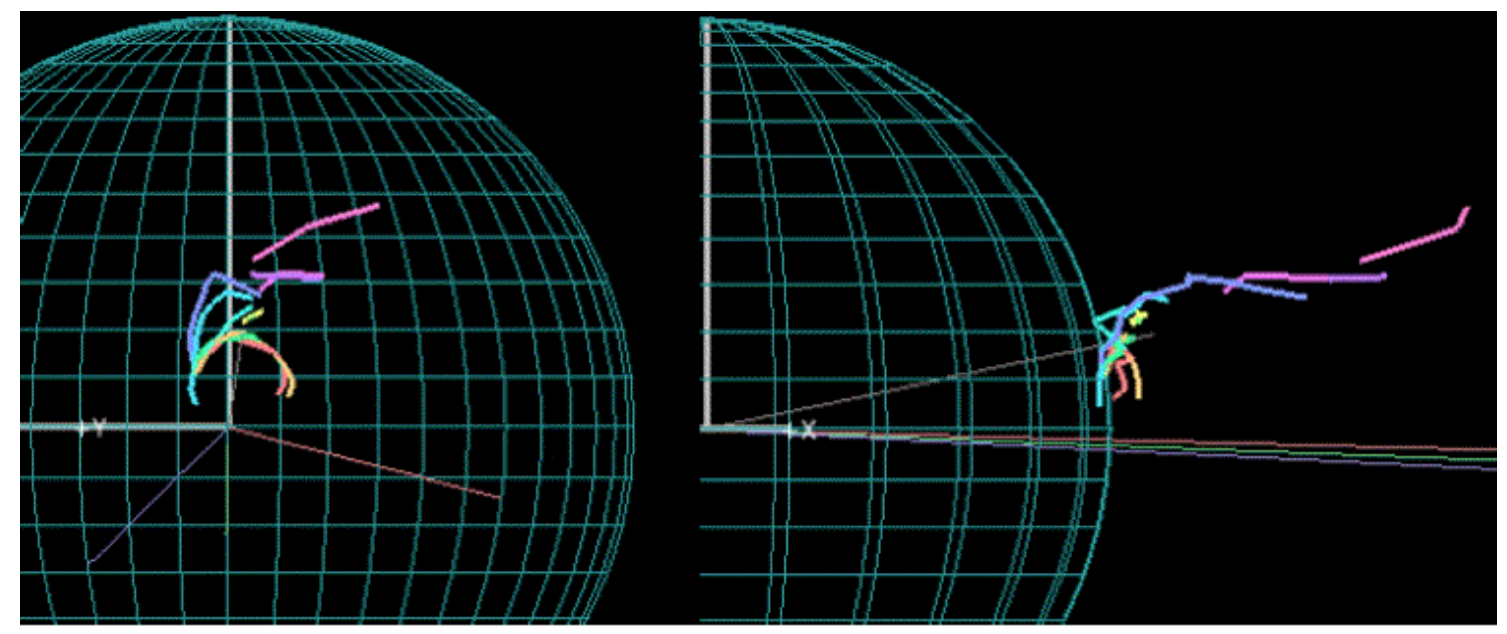

Figure 40: Two views of 3D reconstruction of the 2007 May 19 erupting filament during 11 time intervals, each one rendered with a different color. The filament erupts with the south-west end rising fastest, performing some counter-clockwise rotation (from Liewer et al., 2009). 


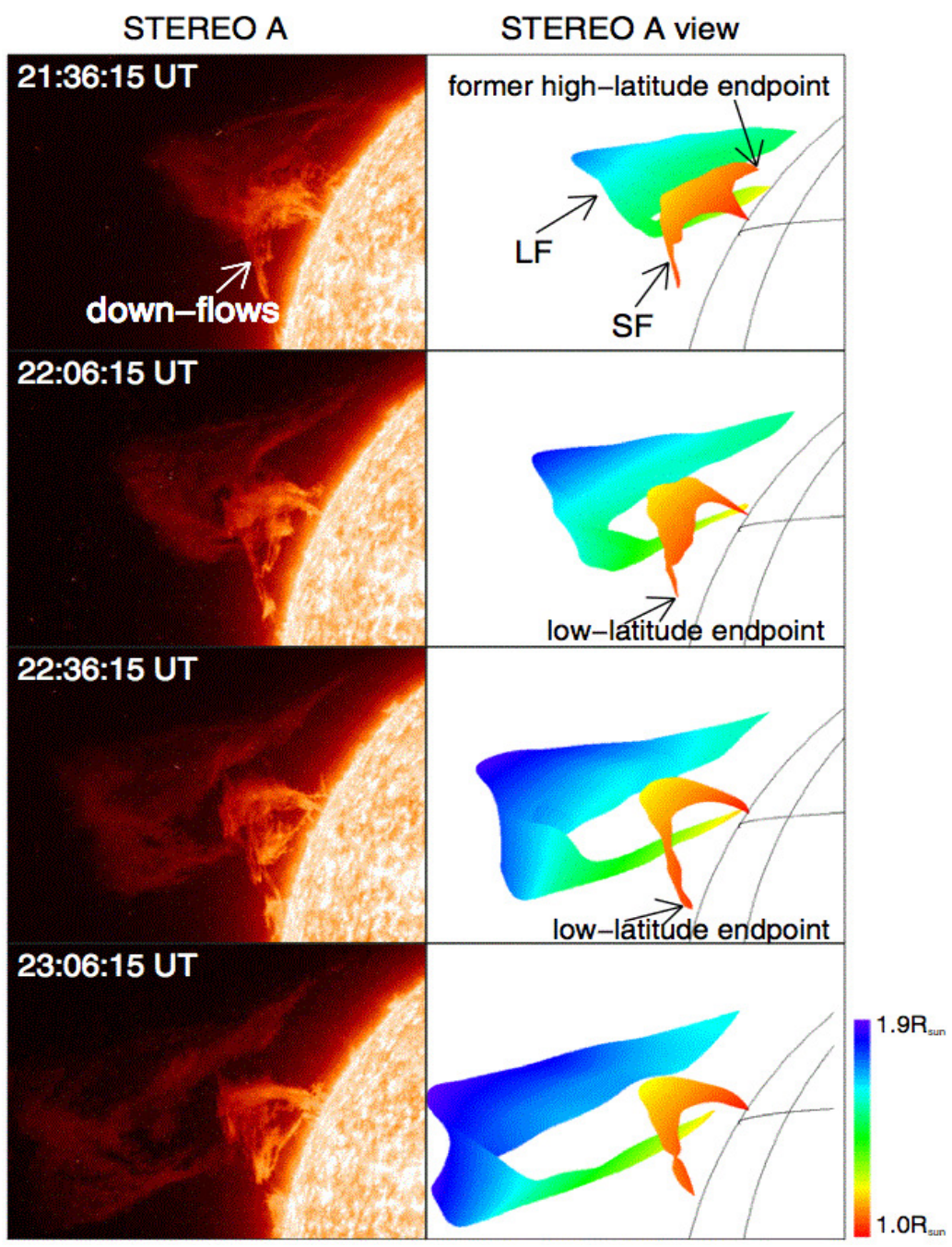

Figure 41: 3D reconstruction of the gradual filament eruption observed on 2009 Sept 26, shown from STEREO/A (left) and reconstructed in 3D (with the z-coordinate in the range of $1.0-1.9 R_{\odot}$ indicated with colors) (from Li et al., 2010a). 


\subsection{Bright points, jets, and plumes}

In this section we summarize stereoscopy of small-scale phenomena in the lower corona, such as point-like features (also called bright points), EUV and soft X-ray jets (collimated plasma outflows mostly along straight channels), and polar plumes (long-lived plasma structures along open field lines in polar coronal holes). These small-scale phenomena may all be triggered by small-scale magnetic reconnection processes in the lower corona that produce plasma heating in small confined loops (EUV bright points) or at the footpoints of open field lines (jets and plumes; Figure 42).

Stereoscopic triangulation of 210 EUV bright points yielded the following heights above the photosphere: $4.4 \pm 1.7 \mathrm{Mm}(304 \AA), 5.1 \pm 1.9 \mathrm{Mm}(171 \AA), 6.1 \pm 1.9 \mathrm{Mm}(284 \AA)$, and $6.7 \pm 2.0 \mathrm{Mm}$ (195 ^) (Kwon et al., 2010). The length of EUV bright points was found to be roughly twice the average height. The findings are consistent with small-scale flaring loops that have a temperature stratification and are heated by magnetic reconnection.

A statistical study of 79 polar jet events observed with STEREO/EUVI and COR-1 demonstrated that the coronal jets are always anchored in underlying small-scale chromospheric bright points, but their morphology revealed symmetric ("Eiffel tower-type"), asymmetric ("lambdatype"), and helical geometries (Nisticò et al., 2009), consistent with magnetic reconnection in tripolar configuration (Figure 42). More than 10,000 jets were identified also in white light with STEREO COR-1 during 2007-2008 (Paraschiv et al., 2010). Some jets are recurring, triggered by a repetitive cycle of magnetic flux cancellation, reconnection, and chromospheric evaporation (Chifor et al., 2008). However, the source of the steady fast solar wind emanating from coronal holes is not all supplied by jets, but also by erupting small-scale loops (He et al., 2010), with temperatures up to $T=12$ MK (Madjarska, 2011). Some recurrent, quasi-homologous jets exhibit a helical geometry and the dynamics of untwisting field lines, which has been modeled (Figure 44) with both a helical rotating current sheet that generates jets and a quasi-steady mode that occurs in a 2D-like current sheet located along the fan between the sheared spines (Pariat et al., 2010). Stereoscopic triangulation of such a polar jet during the initiation phase confirmed the helical geometry and the untwisting mechanism (Patsourakos et al., 2008). Similarly, the unfolding motion of a twisted magnetic flux rope was inferred in a macrospicule, while the associated X-ray jet consisted of a radial outflow (Kamio et al., 2010). A dichotomy of standard jets (Figure 42) and blowout jets (Figure 43) was proposed by Moore et al. (2010), where the non-standard jets undergo a miniature version of the blowout eruption known in major CMEs.

Soft X-ray and EUV observations with Hinode/XRT and STEREO/EUVI demonstrated that $90 \%$ of jets observed in a polar hole were associated with plume haze and $70 \%$ of the jets are followed by polar plumes with a time delay of a few minutes, which suggests that polar jets are precursors of polar plume formations (Raouafi et al., 2008). A time-evolving 3D tomographic reconstruction of polar plumes was attempted with SOHO/EIT images, using the solar rotation and relying on their long lifetime (Barbey et al., 2008). The first stereoscopic triangulation of polar plumes was performed on 10 polar plumes, which showed the superradial expansion in the coronal holes, a density scale height of 1.6-1.8 times the hydrostatic scale height, and outflows of $\lesssim 10 \mathrm{~km} \mathrm{~s}^{-1}$, insufficient to drive the fast solar wind (Feng et al., 2009; Curdt et al., 2008). In contrast, time series analysis of STEREO/EUVI image sequences revealed quasi-periodically driven high-velocity outflows in polar plumes with mean velocities of $135 \mathrm{~km} \mathrm{~s}^{-1}$ at temperatures of $T \approx 0.5-1.5 \mathrm{MK}$, which makes them to an efficient source of heated mass originating in the upper chromosphere and feeding the fast solar wind (McIntosh et al., 2010). Alternative interpretations in terms of acoustic waves, however, cannot be ruled out according to recent debates. 


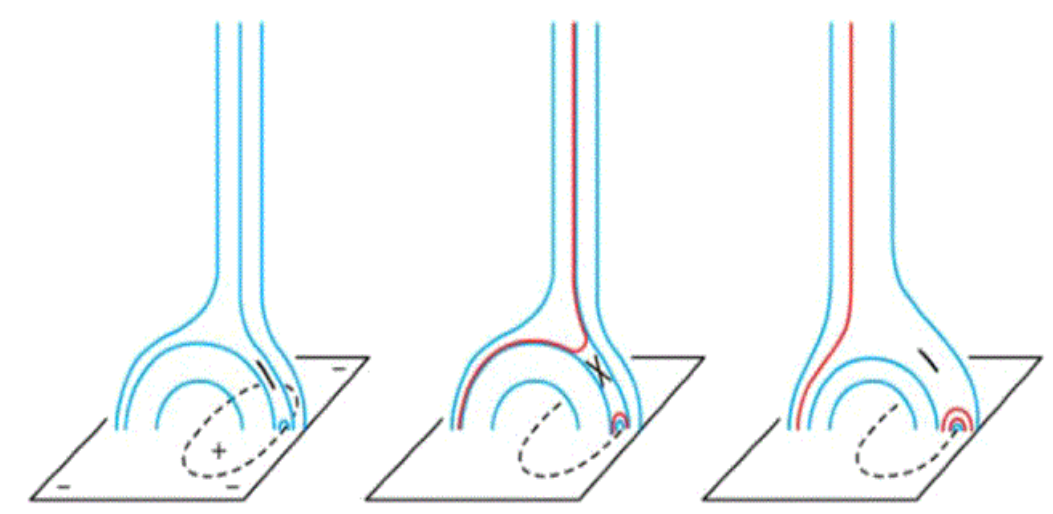

Figure 42: Schematic depiction of tripolar reconnection between a closed-field loop and an open-field line (left), which channels the heated plasma after reconnection (marked with X) upward along the open field line (red line). This tripolar configuration became the standard scenario for coronal jets (from Moore et al., 2010).
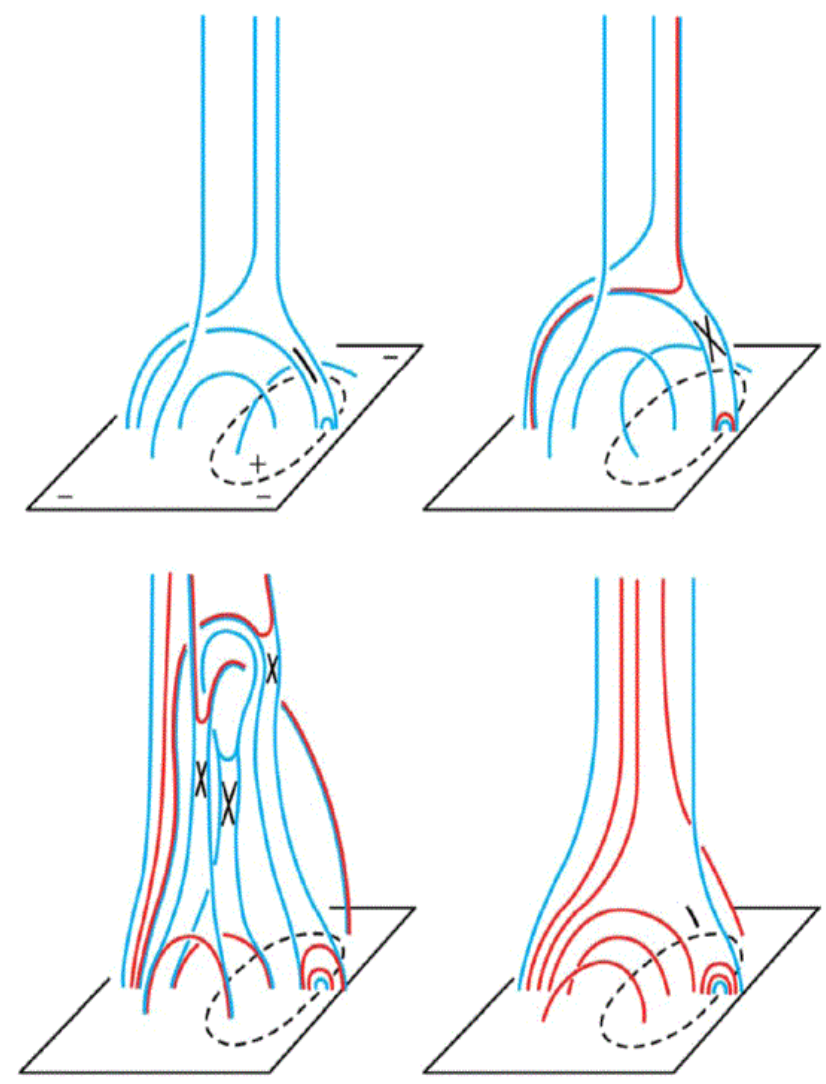

Figure 43: Schematic depiction of the topology, eruption, and reconnection of the magnetic field for a blowout jet. The red field lines demarcate the already reconnected field that contains hot plasma, while the blue field lines indicate the pre-reconnection of not-reconnecting field (from Moore et al., 2010). 


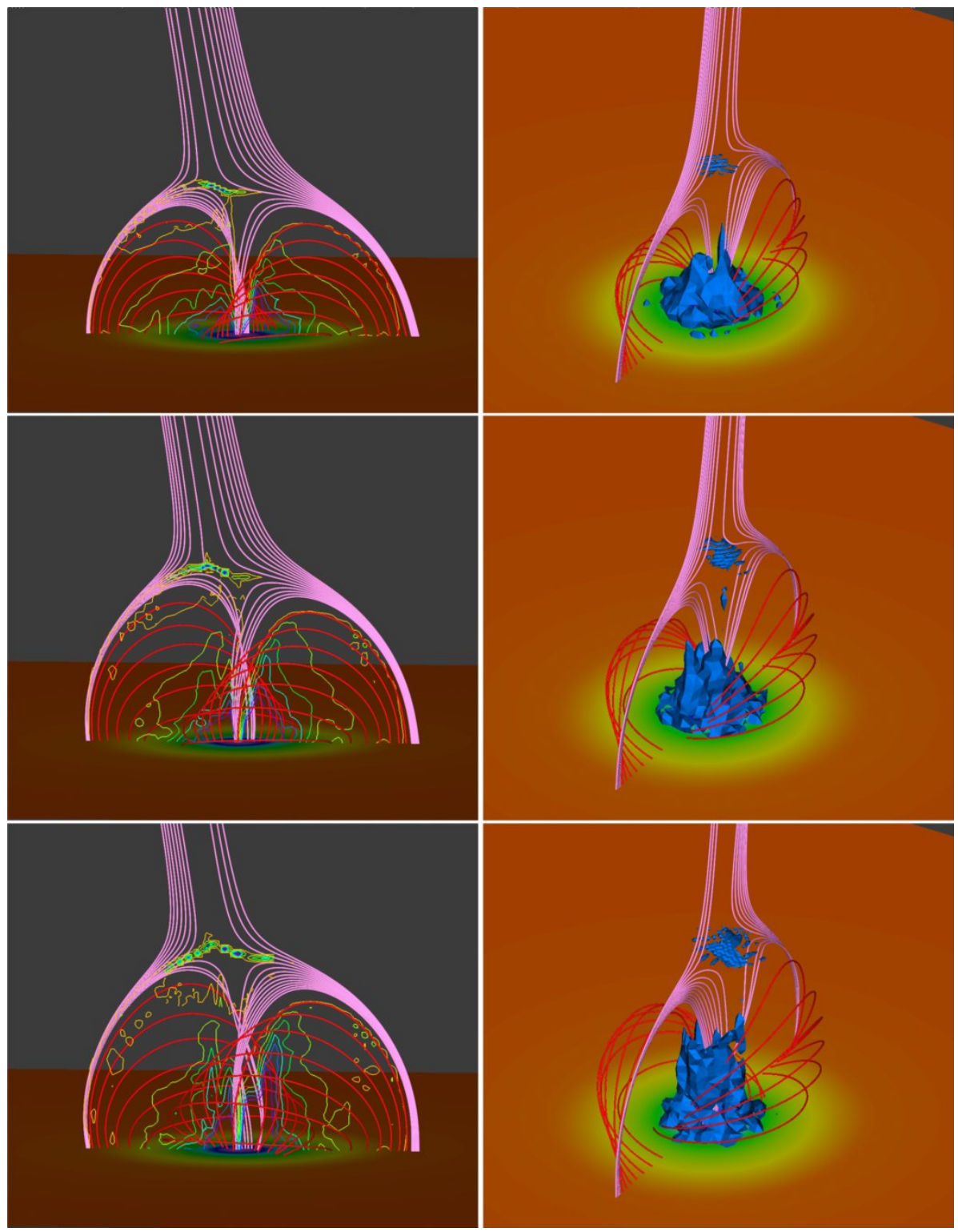

Figure 44: Side view (left) and perspective view (right) of the formation of the current sheet before onset of the first jet (at three different times). The field lines map out the fan surface and fan separatrix (from Pariat et al., 2010). 


\subsection{Solar flares}

Stereoscopic or tomographic 3D reconstructions of solar flares are extremely difficult due to the rapid evolution during the impulsive energy release phase. Even in the postflare phase, loop arcades are gradually expanding and subject to conductive and radiative cooling with constantly changing brightness in each soft X-ray and EUV temperature filter on time scales of minutes. The rapid dynamics excludes any solar rotation based method and allows at best only instantaneous stereoscopy. However, even instantaneous stereoscopy, e.g., with the STEREO/EUVI and SDO/AIA telescopes, are strongly hampered in the impulsive flare phase due to overexposure, pixel bleeding, and the appearance of diffraction patterns from the focal support structure in the telescope aperture. For these reasons, there exists not much stereoscopic literature about solar flares.

Some alternative 3D reconstruction techniques of solar flares have been invented in the preSTEREO era, such as using Doppler-shift measurements of plasma upflows in flare loops to constrain the flare loop inclination angle (Nitta et al., 1999), or using the solar surface as a "Compton mirror" to view precipitating electrons from bottom up, a method called 'stereoscopic electron spectroscopy' (Kontar and Brown, 2006), or dual spacecraft measurements of hard X-rays with RHESSI and type III bursts with WIND in a partially disk-occulted flare (Krucker et al., 2008).
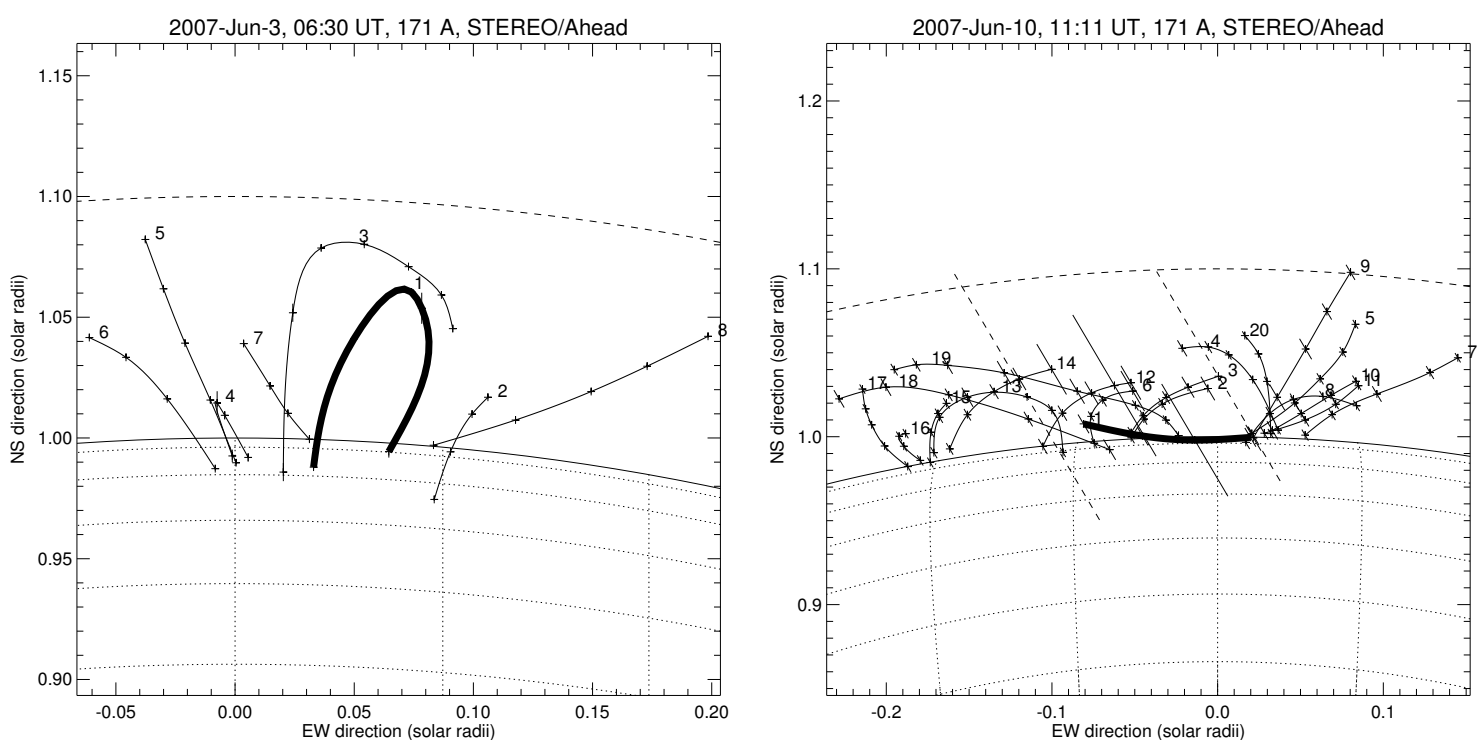

Figure 45: 3D reconstruction of postflare loops of the 2007 Jun 3, 07:50 UT, flare with STEREO/A and B (left), and of the central flaring, non-eruptive filament in the 2007 Jun 10, 11:11 UT, flare (right). Note the difference in altitude for the eruptive flare with post-flare arcade (left) and the non-eruptive flare filament (right) (from Aschwanden et al., 2009b).

A review and a flare catalog of 185 flare events observed with STEREO has been compiled for the first two years of the mission (Aschwanden et al., 2009b), detected by GOES above the C1-level or with RHESSI at $>25 \mathrm{keV}$. CMEs were reported for a third of these events. This flare dataset contains: $79 \%$ events with impulsive EUV emission (coincident with hard X-rays), $73 \%$ show delayed EUV emission from postflare loops and arcades, $24 \%$ represent occulted flares, $17 \%$ exhibit EUV dimming, $5 \%$ show loop oscillations or propagating waves, and at least $3 \%$ show erupting filaments. The stereoscopic view allows us to triangulate a number of individual loops in the flare region, of which we show two examples in Figure 45. The two examples characterize also two different flare categories: (i) eruptive flares that form a postflare arcade below the magnetic 
reconnection region after eruption, usually delayed in EUV with respect to soft X-rays due to the flare plasma cooling (Figure 45, left); and (ii) confined flares that show an impulsive heating phase of a highly-sheared non-eruptive filament, while the overall magnetic configuration pretty much stays intact and unchanged during the entire flare. The altitude of the flaring non-eruptive filament was stereoscopically triangulated to $h=1.8 \pm 2.4 \mathrm{Mm}$, which essentially corresponds to the upper bound of the chromosphere (Figure 45, right). A number of flares appeared to be occulted by one STEREO spacecraft, but in full view by the other spacecraft, which produces two dissimilar EUV time profiles and helps to isolate the fainter coronal emission of a flare.

Other flare studies involving STEREO data include: Magnetic modeling with NLFFF models that localized quasi-circular flare ribbons at the separatrix between open and closed fields ( $\mathrm{Su}$ et al., 2009); correlation of the height-time evolution of CMEs with flare hard X-ray emission (Chen and Kunkel, 2010); STEREO-constrained two-ribbon flare geometry and occultation height of RHESSI and radio observations Krucker et al. (2010); a STEREO-identified twisted helical penumbral filament before an M8.9 flare (Figure 46) (Kumar et al., 2010); evidence of plasmoidlooptop interaction and magnetic inflows during a solar flare and CME eruptive event (Milligan et al., 2010); evidence for internal tether-cutting in a flare and CME event (Raftery et al., 2010); and a correlation between the CME acceleration profile and the energy release rate as measured in hard X-rays with RHESSI (Temmer et al., 2010). In most of these studies, STEREO data were used in a qualitative way to disentangle the 3D flare geometry, while quantitative 3D multi-wavelength modeling that could most suitably done with STEREO (and from triple view points in conjunction with AIA) is still lacking. Ultimately, 3D modeling of flare volumes is necessary to infer accurate values for total flare energies, to determine the 3D fractal dimensions, and to test spatio-temporal scaling laws and powerlaw occurrence frequency distributions in terms of self-organized criticality models (Aschwanden, 2011).
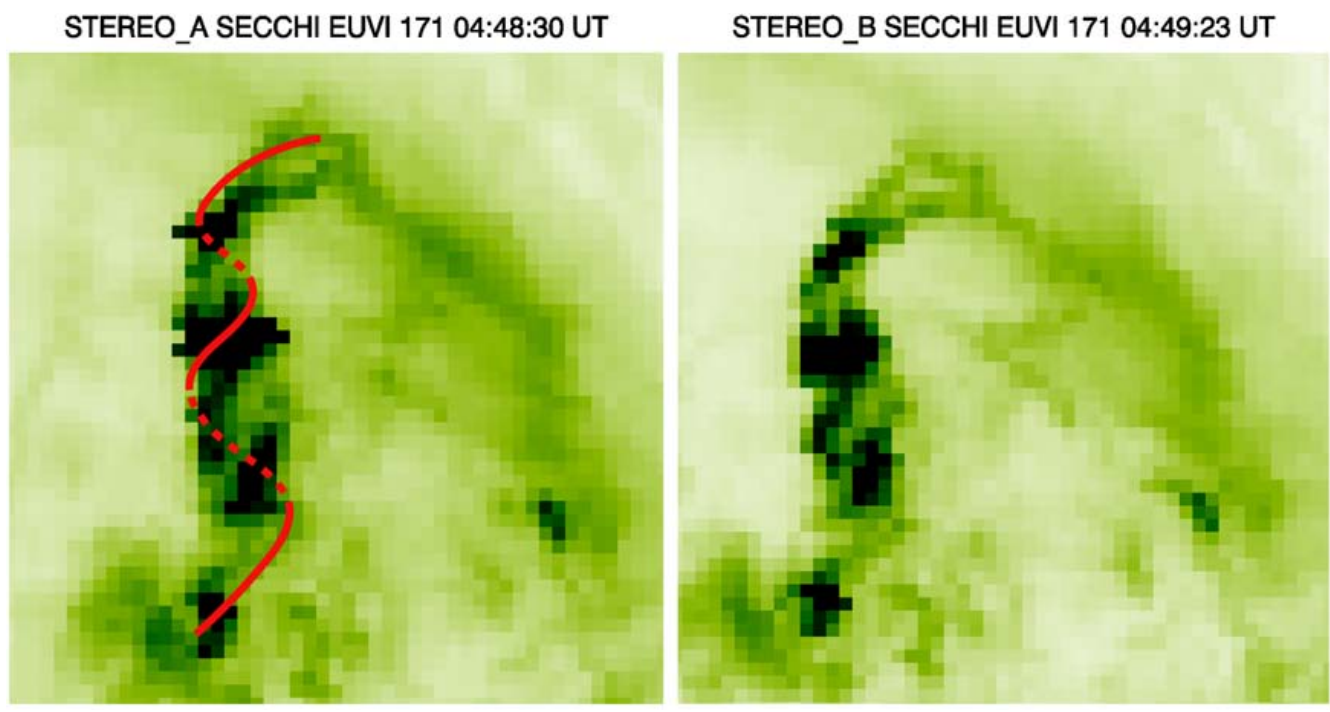

Figure 46: STEREO/EUVI/A and B observations of a twisted helical filament with approximately two turns (red helical line) observed shortly before the M8.9 flare on 2007 Jun 4, 05:08 UT (from Kumar et al., 2010). 


\subsection{CME source regions and EUV dimming}

In this review we limit our scope to stereoscopy and tomography in the solar corona, which includes the manifestations of Coronal Mass Ejections (CMEs) in the source regions, such as EUV dimming and global (EIT) waves, while stereoscopy and tomography of CMEs in the heliosphere and interplanetary space should be reviewed separately, given the massive amount of literature that emerged over the first four years of the STEREO mission. A review on models and observations of CMEs appeared recently in Living Reviews in Solar Physics (Chen, 2011), however, without treatment of stereoscopic and tomographic 3D reconstruction methods. Other recent reviews on CME theoretical models can be found, e.g., in Forbes et al. (2006), Schrijver (2009), or Démoulin and Aulanier (2010).

Manifestations of CMEs in the source region are best visible in EUV and in soft X-rays. There is a wide concensus that both CMEs and flares are interlinked processes of the same magnetic instability in coronal eruptive events. The triggering mechanism of the magnetic instability could be (i) a tether-cutting or flux cancellation mechanism, (ii) shearing motions, (iii) the magnetic breakout model, (iv) an emerging flux triggering mechanism, (v) a flux injection triggering mechanism, or (vi) a kink instability or torus instability (Chen, 2011; Cheng et al., 2010). In all cases, a part of the unstable coronal volume starts to expand and to raise upward, which diminishes the density inside the expanding volume according to,

$$
n_{e}(t)=n_{0}\left(\frac{r(t)}{r_{0}}\right)^{-3}
$$

in the approximation of a spherical CME bubble with initial radius $r_{0}$ and density $n_{0}$. The expansion of the CME bubble may be driven by a Lorentz force, which can cause a near-constant acceleration $a$ during the initial phase, so that the kinematics can be described as,

$$
r(t)=r_{0}+v_{0}\left(t-t_{0}\right)+\frac{1}{2} a\left(t-t_{0}\right) .
$$

The density decrease inside the expanding CME bubble causes a deficit in the emission measure in the corresponding part of the solar corona,

$$
E M(x, y, t)=\int n_{e}^{2}(x, y, z, t) d z=\int n_{0}^{2}\left(\frac{r(t)}{r_{0}}\right)^{-6} d z
$$

which is called $E U V$ dimming. A statistical study that investigated the relationship between EUV dimming (detected with SOHO/CDS) and CME events (detected with SOHO/LASCO) for the time span of 1998-2005 found that 55\% of identified dimming regions are associated with CMEs, while $84 \%$ of the detected CMEs could be tracked back to dimming regions (Bewsher et al., 2008). The mutual correlation might be even higher if dimming regions on the backside of the Sun could be observed. Of course, there are also exceptions, so-called stealth CMEs, which apparently start higher up in the corona and expel so little mass that they cannot be detected in EUV, but are visible in polarized brightness in white-light (Robbrecht et al., 2009). One out of three CMEs during the solar minimum were found to be a stealth CME without coronal signatures, such as dimming, waves, filament eruptions, flares, or post-eruptive arcade on the disk (Ma et al., 2010).

An example of a CME with a bubble-like expansion, observed with STEREO/EUVI/A and B on 2008 Mar 25 is shown in Figure 47. The EUV light curve (diamonds in Figure 47 top panel) shows a massive drop of the total EUV brightness about at the same time when the hard Xray emission of the associated flare peaks (solid curve in Figure 47 top panel), which illustrates the simultaneity of the magnetic instability that causes the launch of the CME, and the coupled magnetic reconnection process that drives the flare with particle acceleration, precipitation (hard 
X-ray bremsstrahlung), and chromospheric evaporation (free-free emission of upflowing heated plasma seen in soft X-rays). A geometric model of a spherical CME bubble expansion and the related EUV dimming calculated from the line-of-sight integral of the emission measure through the solar corona across the CME bubble is shown in Figure 48. The relative dimming in EUV is strongest in the lowest density scale height of the solar corona where most of the expelled mass resides. The 4D modeling of the CME expansion and related EUV dimming of this CME event of 2008 Mar 25 is visualized with a numerical simulation in Figure 49 and is described in more detail in several studies (Aschwanden, 2009b; Aschwanden et al., 2009b; Patsourakos et al., 2010).
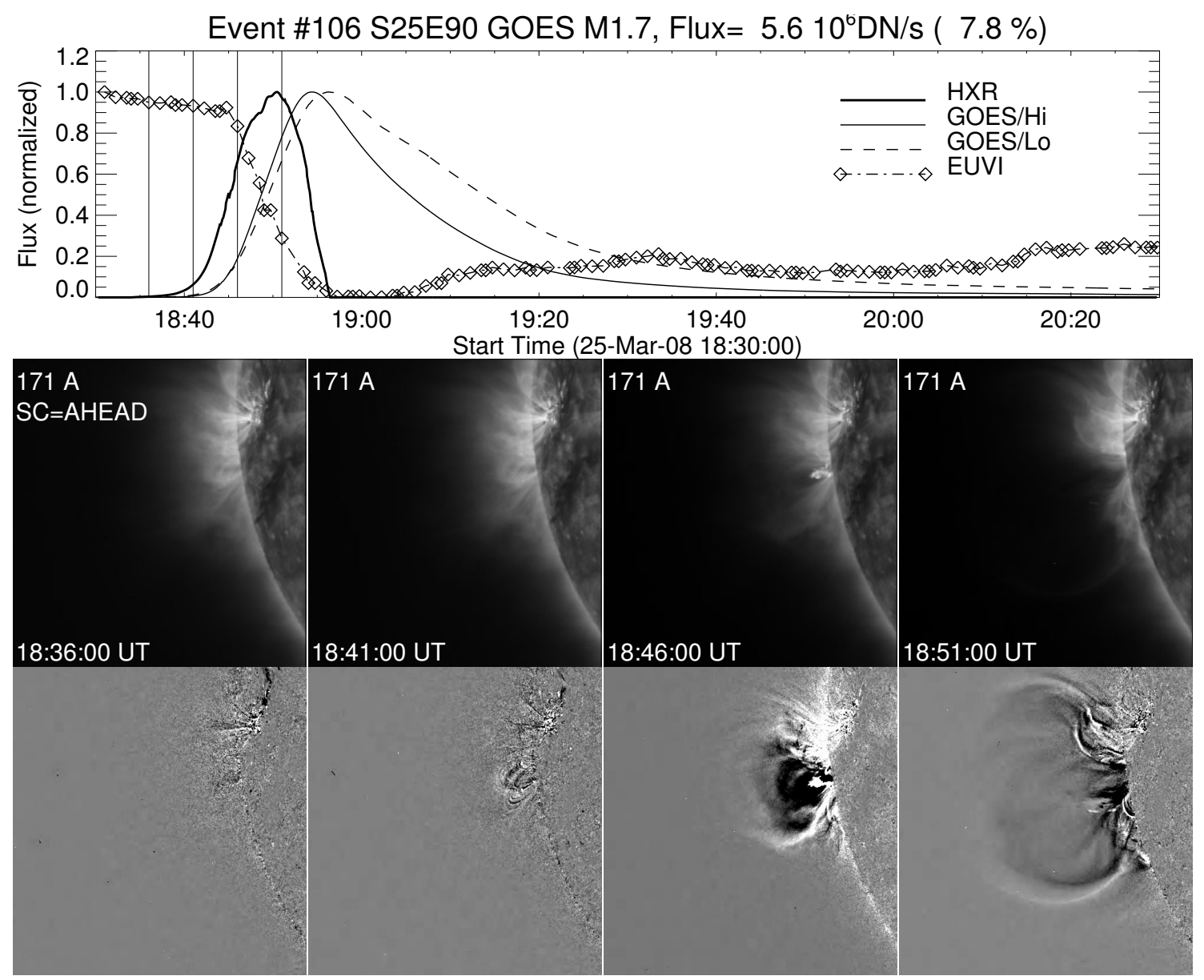

Figure 47: Soft X-ray (GOES/Lo 0.5-4 $\AA$ and 1-8 A, thin curves) and EUV (EUVI/A, diamonds) light curves, time derivative, $d I(t) / d t$ ), of the harder soft X-ray light curve (thick solid line) are shown for the flare/CME event of 2008 Mar 25, 18:30 UT (top panel). Four EUVI/A images (second row) and running difference images (bottom row). Note the strong dimming in the EUV light curve. The diamond symbols mark the times of the EUV images, while the selected images shown below are marked with vertical lines. The peak EUV flux is $F=5.6 \times 10^{6} \mathrm{DN} \mathrm{s}^{-1}$ (or $7.8 \%$ of the total flux). The FOV of the images is 512 EUVI pixels $(\approx 600 \mathrm{Mm}$ ) (from Aschwanden et al., 2009b).

The amount of EUV dimming can quantitatively be used to estimate the mass of a CME. If we characterize the footprint area of a CME with a spherical area with radius $r_{\mathrm{CME}}=R_{\odot} \theta$ and CME angle $\theta$ (latitude or longitude extend in units of radian), and the vertical mass extent with

Living Reviews in Solar Physics

http://www. livingreviews.org/lrsp-2011-5 

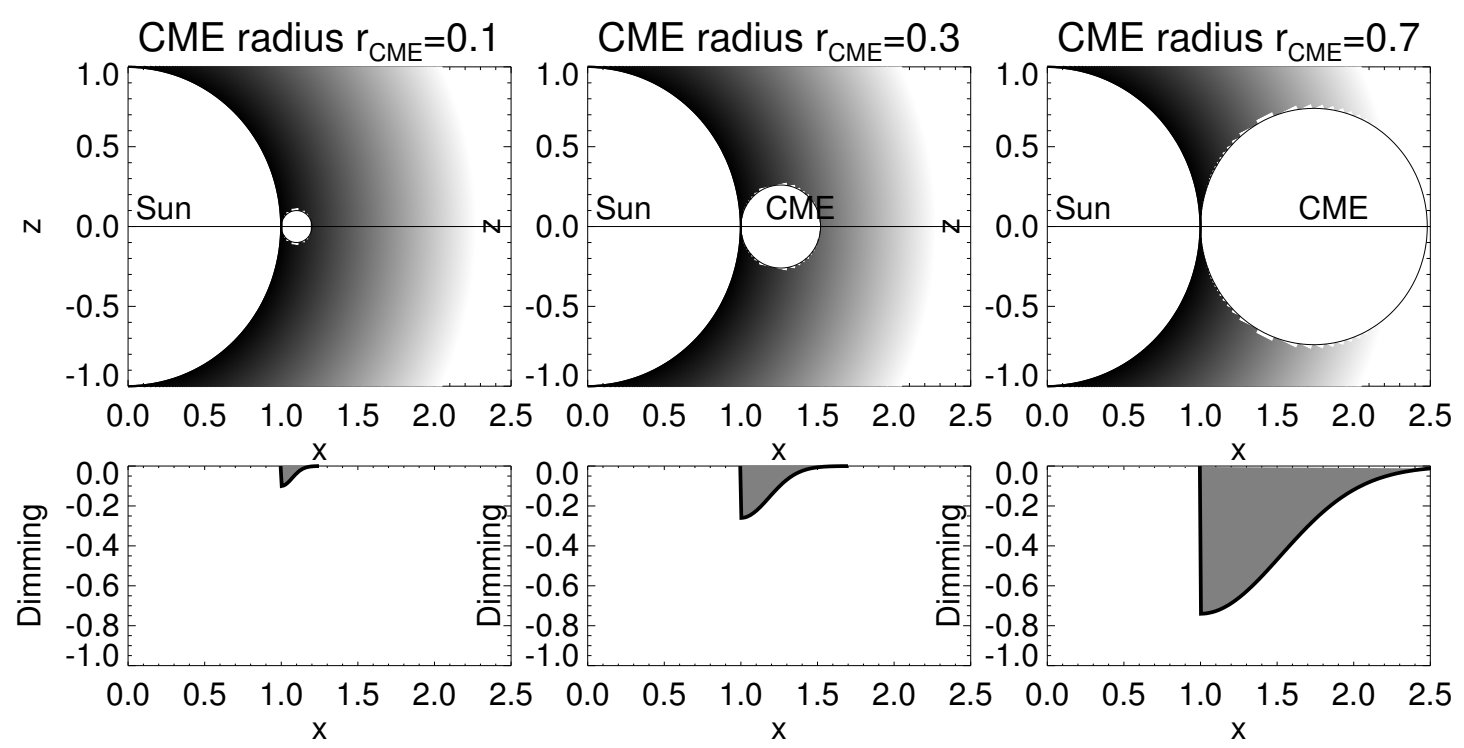

Figure 48: A numerical simulation of adiabatic CME expansion and resulting EUV dimming is shown in the $x$ - $z$ plane for three different times, with $x$ the direction of the CME trajectory and $z$ the line-ofsight direction of the observer. The relative EUV dimming $q_{\text {dimm }}(x, t)$ (normalized to the preflare value) resulting from the LOS-integrated emission measure is shown in the lower panels (from Aschwanden et al., 2009b).

a hydrostatic density scale height $\lambda_{T}$, the original CME source volume is,

$$
V_{\mathrm{CME}}=\left(R_{\odot} \theta\right)^{2} \lambda_{T},
$$

which leads to the following CME mass estimate,

$$
m_{\mathrm{CME}}=V_{\mathrm{CME}} n_{e} m_{p}=1.16 \times 10^{15}\left(\frac{\theta}{10^{\circ}}\right)^{2}\left(\frac{T}{1 \mathrm{MK}}\right)\left(\frac{n_{\mathrm{base}}}{10^{9} \mathrm{~cm}^{-3}}\right)[g] .
$$

This yields for a typical base density of $n_{\text {base }}=10^{9} \mathrm{~cm}^{-3}$, a coronal temperature of $T=1.0 \mathrm{MK}$, and an opening angle range of $\theta \approx 3^{\circ}-30^{\circ}$, CME masses in the range of $m_{\mathrm{CME}} \approx 10^{14}-10^{16} \mathrm{~g}$. More accurate estimates could easily be obtained by combining the coronal EUV filters in order to have more comprehensive temperature coverage (Robbrecht and Wang, 2010; Landi et al., 2010), e.g., $T \approx 0.7-2.7$ MK for the three STEREO/EUVI filters in 171,195 , and $284 \AA$. Applying Eq. (48) to the 2008 Mar 25 flare, where the EUV dimming extends over an opening angle of $\theta \approx 13^{\circ}$, we estimate a CME mass of $m_{\mathrm{CME}} \approx 2 \times 10^{15} \mathrm{~g}$, which includes the CME plasma in the temperature range of $T \approx 0.7-1.3 \mathrm{MK}$ and, thus, represents a lower limit for the entire CME mass. More detailed geometric modeling for arbitrary locations in the solar corona and combining the emission measures from different temperature filters in $6 \mathrm{CME}$ events yielded CME masses in the range of $m_{\mathrm{CME}}=(2-7) \times 10^{15} \mathrm{~g}$, which agreed between the two STEREO/A and B spacecraft $\left(m_{A} / m_{B}=1.3 \pm 0.6\right)$ as well as with white-light measurements by COR2 $\left(m_{\mathrm{EUVI}} / m_{\mathrm{COR} 2}=1.1 \pm 0.3\right)$ (Aschwanden et al., 2009a). Previous CME masses were determined by assuming the propagation direction to be aligned with the plane-of-sky (Vourlidas et al., 2010), but improved values that are corrected for the (stereoscopically triangulated) "true" 3D propagation direction have been derived in the latter comparison (Colaninno and Vourlidas, 2009), based on the assumption that both STEREO spacecraft measure equal CME masses. 

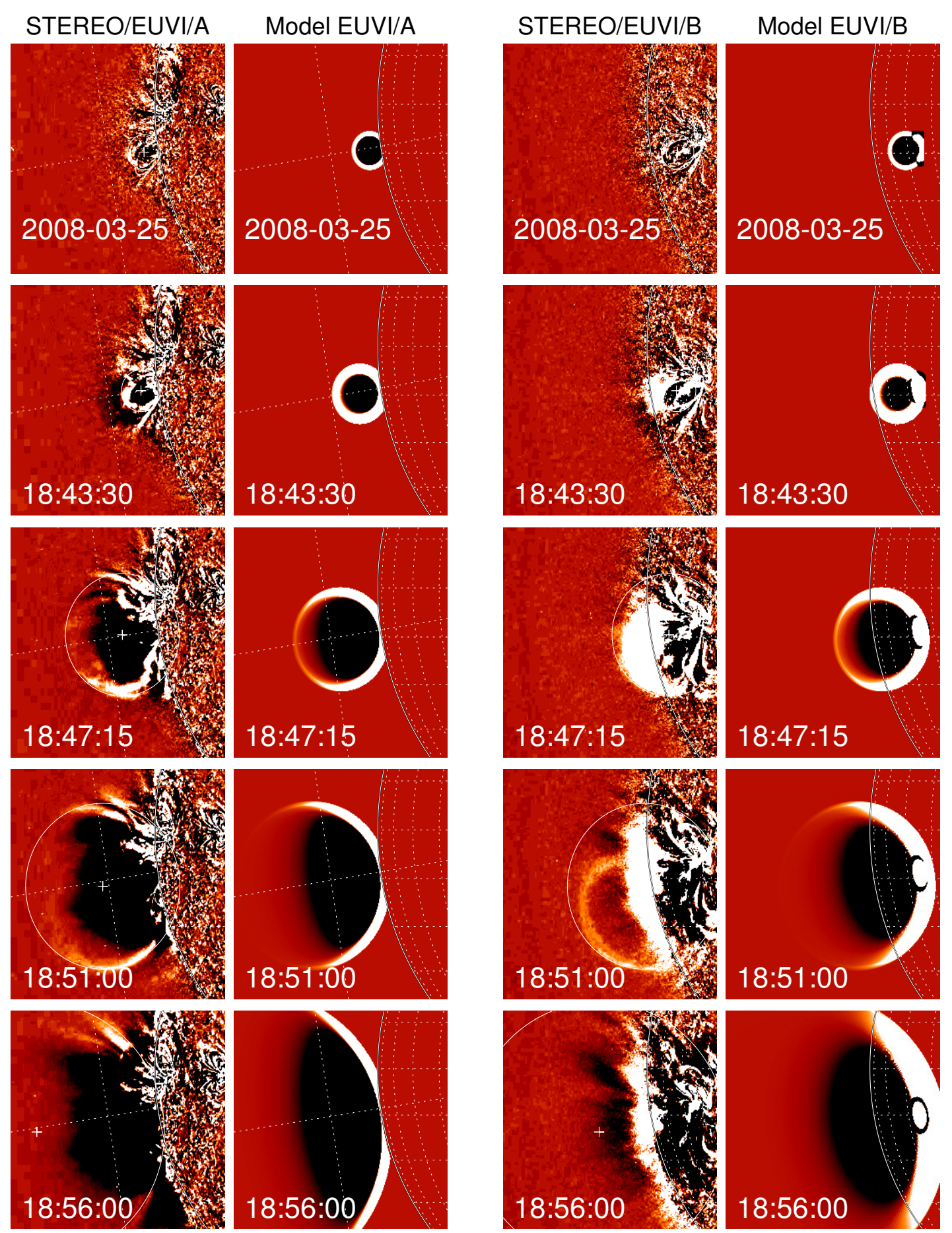

Figure 49: Comparison of observed and simulated EUVI base-difference images at 5 times for the observations of STEREO/A $171 \AA$ (left two columns) and STEREO/B $171 \AA$ (right two columns). The pre-CME image at 18:36 UT was subtracted in these base-difference images (from Aschwanden et al., 2009b). 
A numerical simulation of a coronal wave - CME - dimming event (2009 Feb 13) was carried out when the STEREO spacecraft were in quadrature, which provided a top-down as well as a side-view of the expanding CME (Cohen et al., 2009). STEREO quadrature observations also revealed that the coronal dimming occurs before the chromospheric eruption, indicating that the removal of the overlying coronal magnetic field is the trigger in miniature CMEs (Innes et al., 2010). Sequential (homologous) CME events may cause "double dimmings" (Li et al., 2010b). EUV dimming may occur at two footpoint locations of an eruptive loop, especially in cases with emerging flux trigger mechanisms (Zheng et al., 2011). The detection and measurement of coronal EUV dimming regions can now be conducted with automated algorithms (Attrill and Wills-Davey, 2010). It would be interesting to compare the automatically detected dimming areas from the two STEREO spacecraft and to stereoscopically triangulate the altitude of the centroids of the dimming areas, which is expected to correspond to a half density scale height.

\subsection{Global coronal waves}

Global coronal waves (originally discovered with SOHO/EIT) propagate concentrically outward of a CME launch site, globally over the solar surface, with typical speeds of $\approx 170-350 \mathrm{~km} \mathrm{~s}^{-1}$. Interpretations of these CME-triggered global waves include: (i) fast-mode MHD magnetoacoustic waves, (ii) successive stretching of magnetic field lines, (iii) successive magnetic reconnection (Attrill et al., 2007), (iv) current shell model, or (v) slow-mode wave or soliton wave model (see review by Chen, 2011, and references therein). Let us review what stereoscopic observations contributed to a better understanding of these global coronal waves.
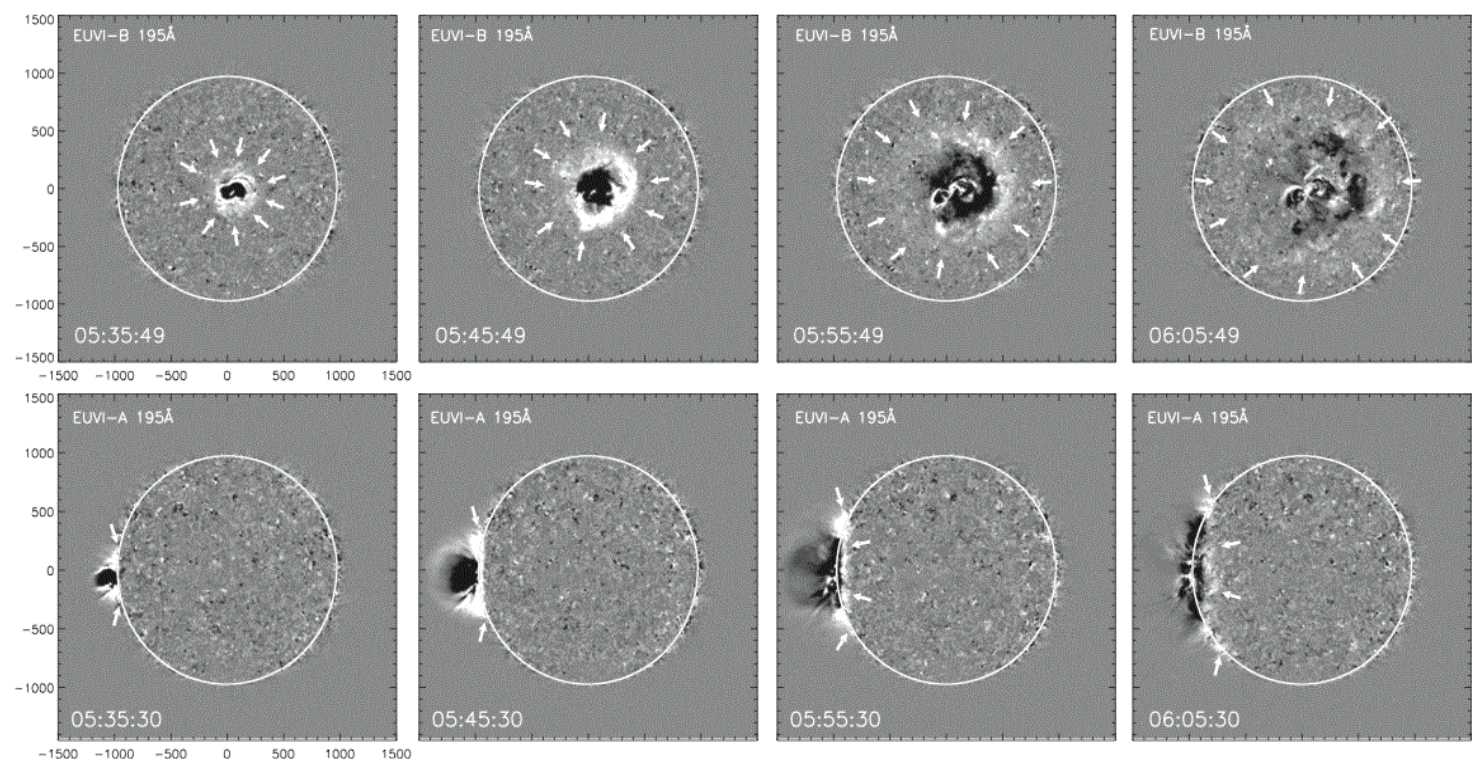

Figure 50: Sequence of median-filtered running difference images recorded in the EUVI $195 \AA$ channel with a cadence of 10 minutes. The coronal wave (outlined by arrows) is observed on-disk in STEREO/B (top) and on the limb in STEREO/A (bottom) (from Kienreich et al., 2009).

The kinematics of global coronal waves were measured with STEREO/EUVI in all four passbands $(304,171,195,284 \AA)$ and it was found that the difference in speeds are partly due to different cadences, but are essentially consistent with an impulsively generated fast-mode magnetoacoustic wave (Long et al., 2008). High-cadence EUVI observations of the 2007 May 19 event 
reveal a deceleration that is indicative of a freely propagating large-amplitude MHD wave (Veronig et al., 2008). The reflection of a propagating EUV disturbance off a coronal hole boundary was considered as a confirmation of its true wave nature (Gopalswamy et al., 2009), and has been numerically simulated with an MHD code (Schmidt and Ofman, 2010). Hinode/XRT and STEREO observations of a diffuse coronal wave observed near the limb show that the core coronal dimmings map to the core of the CME, secondary coronal dimmings map to the CME cavity, and the diffuse coronal wave maps to the outermost edge of the expanding CME shell (Attrill et al., 2009). The numerical simulations of a CME dimming and global wave event observed with STEREO in quadrature confirmed that the global wave front is made of a mass density (rather than a temperature) enhancement and propagates at a height of $\approx 1.1 R_{\odot}$ (Cohen et al., 2009). Ideal STEREO quadrature observations of a wave's initiation at disk center for STEREO/B and exactly at the limb for STEREO-A show the 3D structure of global wave most clearly (Figure 50), with a constant propagation velocity of $\approx 236 \pm 16 \mathrm{~km} \mathrm{~s}^{-1}$ (close to the fast magnetosonic speed in the quite corona) at a height of $\approx 80-100 \mathrm{Mm}\left(\approx 1.11-1.14 R_{\odot}\right)$ (Kienreich et al., 2009). Confusion in tracking and identifying a global coronal wave arises also from cospatial projection effects (of the column depth) of the expanding CME bubble, which may expand at a different speed in the upper corona than the global wave in the lower corona, especially for halo CMEs (Ma et al., 2009). Therefore, STEREO quadrature observations are the most unambiguous method to separate the CME structure from the EUV wave and confirms the fast-mode MHD wave interpretation (Patsourakos and Vourlidas, 2009; Patsourakos et al., 2009). However, the global EUV wave is not always restricted to a low coronal altitude. In some cases a clear dome-shaped 3D surface can be seen (Veronig et al., 2010), which probably coincides with the leading edge of the CME (Figure 51). Arguments against the fast-mode MHD wave interpretation was mounted by STEREO observations of strongly variable propagation speeds (Zhukov et al., 2009), while a unified scenario was proposed that includes both a wave-like component moving at the fast magnetosonic speed and a coherent driven compression front related to the eruptive CME bubble (Downs et al., 2011). 

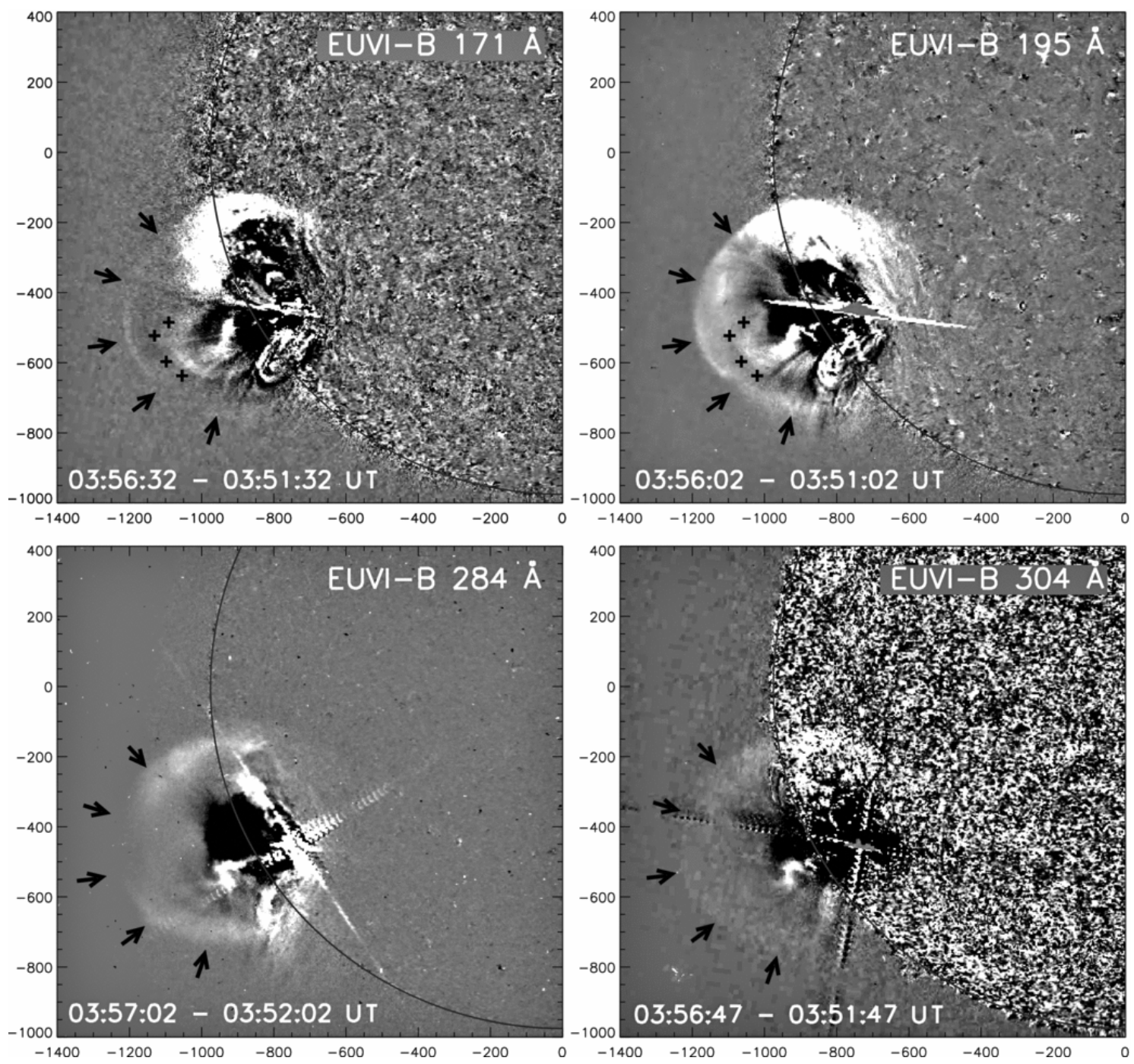

Figure 51: Running difference images (in 5-min intervals) of the dome-shaped EUV wave front as observed with the EUVI/B channels in the 171, 195, 284, and $304 \AA$ wavelengths. Arrows outline the wave dome, crosses indicate the erupting CME loops inside the dome (from Veronig et al., 2010). 


\section{Summary}

Most physical models of solar phenomena require the knowledge of the 3D geometry of the object, which instigates reliable 3D reconstruction methods, such as stereoscopy and tomography. Stereoscopy requires a view from two different vantage points, which is now most suitably provided by the dual STEREO spacecraft, but has also been applied to other multi-spacecraft observations before, or using the solar rotation for quasi-static phenomena. Tomography is a technique to combine imaging informations from multiple slices (with different aspect angles) into a 3D density distribution, which can only be carried out in a minimal way in the solar case, by using two or three spacecraft views, unless the solar rotation is used and the object remains quasi-stationary during the time interval of $3 \mathrm{D}$ reconstruction. We see that both the stereoscopy and tomography method share the trade-offs of (i) stationarity for solar rotation based methods, or (ii) minimal aspect angle coverage for simultaneous observations, due to the low number of available spacecraft. Nevertheless, stereoscopic and tomographic 3D reconstruction methods became more and more refined by taking stock of auxiliary geometric information from magnetic field models and opacity models, methods that also called "magnetic stereoscopy" or "frequency tomography". Also solar rotation based methods became more sophisticated by incorporating the dynamics of solar phenomena to first order, such as the "dynamic stereoscopy" or "Kalman filter tomography" methods. Of course, the biggest boost came from the launch of the STEREO mission in 2006, which produced a tenfold increase of publications dealing with solar stereoscopy and tomography. In this review here we focused on stereoscopic and tomographic reconstruction of phenomena in the solar corona, but omitted applications in the heliosphere (interplanetary CMEs) or in the solar interior (helioseismic tomography).

The stereoscopic and tomographic 3D reconstruction methods are wavelength-dependent and require a proper treatment of the opacity in each wavelength. Observations in EUV and soft X-rays involve optically thin free-free emission and have an additive characteristic of the squared electron density integrated along each line-of-sight. Observations in white-light are governed by Thompson scattering and scale in brightness proportional to the (scattering) electron distribution along a lineof-sight. Observations at radio wavelengths can be dominated by free-free emission in the opticallythin regime, or by gyroresonance emission in the optically-thick regime and, thus, requires a 3D density and temperature model for each line-of-sight. Classical stereoscopy represents a simple geometric triangulation from two view points to obtain the $3 \mathrm{D}$ coordinates of a point-like or curvilinear structure, but solar applications are somewhat more complicated, since the intersection point is defined by the emission measure-weighted brightness distribution for optically-thin emission, or by an opaque surface for optically-thick emission.

Let us summarize the basic physical results that have been or could be obtained for coronal observations by means of stereoscopic or tomographic analysis methods:

1. Large-scale corona: Tomography yields the $3 \mathrm{D}$ density distribution $n_{e}(l, b, r)$ in the corona in a typical height range of $r \approx 1.0-2.5 R_{\odot}$, which can be rendered as a synoptic map $n_{e}(l, b)$ at a given height level $r$, or in form of radial density scale heights, $n_{e}(r)=n_{0} \exp (-h / \lambda)$, for every heliographic position $(l, b)$. Synoptic density maps are useful to characterize the source function of the solar wind, while local density scale heights can verify hydrostatic gravitational stratification, or in deviating cases, serve as diagnostic of non-equilibrium hydrodynamic processes.

2. Coronal streamers: Tomography of the large-scale corona usually reveals (long-lived) streamers as most prominent features. Detailed 3D reconstructions of the streamer belt may reveal the folds of the interplanetary current sheet, double plasma sheets and triple current sheets and, thus, provides essential information on the lower boundary for global magnetic field models in the corona and interplanetary space.

Living Reviews in Solar Physics

http://www. livingreviews . org/lrsp-2011-5 
3. Active regions: The $3 \mathrm{D}$ density $n_{e}(l, b, h)$ and temperature distribution $T_{e}(l, b, h)$ could be reconstructed with tomography using the two STEREO spacecraft with triple EUV temperature filters, applying stereoscopic triangulation and differential emission measure (DEM) modeling to a skeleton of some 100 loops, and interpolating the 3D magnetic field in a spacefilling volume. Such a 3D model can provide the DEM distribution in any subvolume of an active region, which can be used to localize and track the heating energy input as a function of space and time.

4. Coronal loops: Many coronal loops can be stereoscopically triangulated, yielding the 3D coordinates $[x(s), y(s), z(s)]$ along the loop length coordinate $s$. DEM modeling of the loops can then be carried out straightforwardly after suitable background subtraction, yielding the density $n_{e}(s)$ and temperature profile $T_{e}(s)$ along the loop. This information can be used (i) to determine the hydrostatic density scale height, which depends on the loop plane inclination angle; (ii) to model the 1D hydrodynamic evolution of loops, as a function of the spatio-temporal heating function and conductive and radiative cooling processes, yielding diagnostics of (RTV) equilibrium and dynamic heating processes; and (iii) to test theoretical (potential, linear, and nonlinear force-free) magnetic field models.

5. MHD loop oscillations: MHD fast kink-mode oscillations show a lateral displacement of the loop positions $[x(s, t), y(s, t), z(s, t)]$ that can be stereoscopically triangulated as a function of the loop length coordinate $s$ and time $t$, yielding geometric information on the orientation of the oscillation loop plane (from horizontal to vertical), the coplanarity, the circularity, and helicity of oscillating loops. This dynamic spatio-temporal information constrains the polarization of the kink mode, torsional modes, and asymmetries and the time dependence of the exciter.

6. MHD waves in loops: Stereoscopic triangulation of loops or fans that contain propagating waves, such as MHD (acoustic) slow-mode waves, yields the absolute spatial direction of the propagating waves and, thus, allows us to correct the observed (projected) wave speeds. This method permitted to measure the true phase speed of sound waves and the temperature of the wave guide, independent of DEM modeling with multi-temperature filters.

7. Erupting filaments and prominences: The 3D trajectory of erupting filaments can be stereoscopically triangulated, which yields information on the asymmetry and timing of the magnetic destabilization process, the speed, acceleration, and driving forces of the erupting filament.

8. Bright points, jets, and plumes: Stereoscopic triangulation of features in small-scale phenomena such as bright points, jets, and plumes, reveals their height in the solar corona, the topology of the magnetic reconnection process (i.e., dipolar, tripolar, quadrupolar, nullpoint, fan surface, fan separatrix, helical, or twisted configuration). The verification of their spatial location at the bottom of the solar corona puts also solid constraints on the spatial distribution of the coronal heating function. Moreover, the importance of small-scale (such as nanoflares) versus large-scale phenomena (such as large flares and CMEs) can only be decided based on the occurrence frequency distribution of their energies, which requires accurate measurements of their $3 \mathrm{D}$ volume.

9. Solar flares: Stereoscopic triangulation during solar flares is somewhat more difficult due to high brightness contrast, saturation, and diffraction pattern effects in EUV and soft X-ray images, but is feasible for a part of the flare loops. The brightest structures during flares turned out to be low-lying non-eruptive filaments in confined flares, or high-lying, expanding postflare loops in eruptive flares, which are two types of flares that can be distinguished 
by stereoscopic triangulation. Stereoscopy plays also an important role in partially occulted flares, where the flaring emission can be differentiated as a function of the altitude (e.g., coronal hard X-ray sources are often only visible when the bright footpoint emission is occulted). Stereoscopically-aided 3D reconstruction of flare regions should yield accurate measurements of the thermal energy (which scales with the flare volume). Flare energies are the most relevant quantity to establish physical scaling laws and the powerlaw-like occurrence frequency distributions, which are the hallmark of complex nonlinear dissipative systems in the state of self-organized criticality.

10. CME source regions and EUV dimming: The most prominent manifestation of CMEs in the lower corona is the EUV dimming, whose location constraints the propagation direction of the CME, whose footprint area yields the volume and mass of CMEs, and whose temporal evolution yields the speed, acceleration, and force that drives the CME. Spatio-temporal 4D modeling of the expanding CME structure (e.g., bubble, ice-cone, flux rope) reveals the magnetic topology of the instability (e.g., tether-cutting, shearing, break-out, kink, or torus instability) and, thus, can reveal the physical mechanism that leads to a CME and/or flare.

11. Global coronal waves: Stereoscopic observations, especially in quadrature, reveal the spatial relationship between the expanding CME structure and the associated global coronal waves that propagate concentrically over the solar surface. The geometric height of the propagating wave, as well as the magnetic field along the trajectories, can verify the physical nature in terms of the local magneto-acoustic phase speed of the wave.

Stereoscopy and tomography are just two special types of 3D reconstruction methods, but we can envision more general methods based on parameterized $3 \mathrm{D}$ or $4 \mathrm{D}$ physical models that can be forward-fitted to observations. Forward-fitting of such physical models to observations from multiple spacecraft, and using multiple temperature filters, yields even more powerful constraints, and represents an even cleaner way to deal with the underlying opacity effects in each wavelength than simple inversions of stereoscopically triangulated depth or line-of-sight coordinates. Forwardfitting of parameterized physical models should also yield more accurate 3D density models of the solar corona than direct tomographic inversion methods, which are extremely under-constrained (with only two or three slices) in the case of solar observations. We anticipate that forward-fitting of $3 \mathrm{D}$ or $4 \mathrm{D}$ physical models to multiple spacecraft data will still be called "stereoscopic" or "tomographic" methods in future, but we should be aware that the meaning of these terms progressively deviates from the original definition of simple stereoscopic triangulation and tomographic inversion. We anticipate powerful results from combined modeling of triple spacecraft data from STEREO and SDO/AIA. 


\section{Acknowledgements}

The author thanks Jean-Pierre Wuelser, Nariaki Nitta, Jim Lemen, and Anne Sandman from the SECCHI/EUVI team for constructive discussions and support. We acknowledge also constructive and helpful comments by two anonymous referees that helped to improve the paper. This research has made extensive use of NASA's Astrophysics Data System (ADS) Bibliographic Services. This work is supported by the NASA STEREO under NRL contract N00173-02-C-2035. The STEREO/ SECCHI data used here are produced by an international consortium of the Naval Research Laboratory (USA), Lockheed Martin Solar and Astrophysics Lab (USA), NASA Goddard Space Flight Center (USA), Rutherford Appleton Laboratory (UK), University of Birmingham (UK), Max-Planck-Institut für Sonnensystemforschung (Germany), Centre Spatiale de Liège (Belgium), Institut d'Optique Théorique et Applique (France), and Institute d'Astrophysique Spatiale (France). The USA institutions were funded by NASA; the UK institutions by the Science \& Technology Facility Council (which used to be the Particle Physics and Astronomy Research Council, PPARC); the German institutions by Deutsches Zentrum für Luft- und Raumfahrt e.V. (DLR); the Belgian institutions by Belgian Science Policy Office; the French institutions by Centre National d'Etudes Spatiales (CNES), and the Centre National de la Recherche Scientifique (CNRS). The NRL effort was also supported by the USAF Space Test Program and the Office of Naval Research. 


\section{References}

Abramenko, V., Yurchyshyn, V., Linker, J., Mikić, Z., Luhmann, J. and Lee, C.O., 2010, "LowLatitude Coronal Holes at the Minimum of the 23rd Solar Cycle", Astrophys. J., 712, 813-818. [DOI], [ADS], [arXiv:1002.1685 [astro-ph.SR]] (Cited on page 28.)

Airapetian, V., Ofman, L., Sittler, E.C. and Kramar, M., 2011, "Probing the Thermodynamics and Kinematics of Solar Coronal Streamers", Astrophys. J., 728, 67. [DOI], [ADS] (Cited on page 32.)

Alexander, D., Gary, G.A. and Thompson, B.J., 1998, "Analysis of Active Regions via 3D Rendering Techniques", in Three-Dimensional Structure of Solar Active Regions, 2nd Advances in Solar Physics Euroconference, (Eds.) Alissandrakis, C.E., Schmieder, B., vol. 155 of ASP Conference Series, pp. 100-104, Astronomical Society of the Pacific, San Francisco. [ADS] (Cited on page 26.)

Alissandrakis, C.E., Gontikakis, C. and Dara, H.C., 2008, "Determination of the True Shape of Coronal Loops", Solar Phys., 252, 73-87. [DOI], [ADS] (Cited on page 26.)

Altschuler, M.D., 1979, "Reconstruction of the global-scale three-dimensional solar corona", in Image reconstruction from projections: Implementation and applications, (Ed.) Herman, G.T., vol. 32 of Topics in Applied Physics, pp. 105-145, Springer, Berlin; New York. [DOI], [ADS] (Cited on pages 6, 15, and 31.)

Artzner, G.E., Gosain, S. and Schmieder, B., 2010, "A Technique for Removing Background Features in SECCHI - EUVI He II $304 \AA$ Filtergrams: Application to the Filament Eruption of 22 May 2008", Solar Phys., 262, 437-447. [DOI], [ADS], [arXiv:1001.4884 [astro-ph.SR]] (Cited on page 58.)

Aschwanden, M.J., 1995, "Imaging, Stereoscopy, and Tomography of the Solar Corona in Soft XRays and Radio", in Coronal Magnetic Energy Releases, Proceedings of the CESRA Workshop held in Caputh/Potsdam, Germany, 16 - 20 May 1994, (Eds.) Benz, A.O., Krüger, A., vol. 444 of Lecture Notes in Physics, pp. 13-34, Springer, Berlin; New York. [DOI], [ADS] (Cited on pages 9,17 , and 33.)

Aschwanden, M.J., 2005, Physics of the Solar Corona: An Introduction with Problems and Solutions, Springer-Praxis Books in Geophysical Sciences, Springer; Praxis, Berlin; New York; Chichester, 2nd edn. [ADS], [Google Books] (Cited on pages 15, 37, 38, and 41.)

Aschwanden, M.J., 2009a, "Hydrodynamic Modeling of Coronal Loops with Hinode and STEREO", in The Second Hinode Workshop Science Meeting: Discovery - Toward Understanding, Proceedings of a meeting at the National Center for Atmospheric Research, Boulder, Colorado, USA, held 29 September - 3 October 2008, (Eds.) Lites, B., Cheung, M., Magara, T., Mariska, J., Reeves, K., vol. 415 of ASP Conference Series, pp. 234-240, Astronomical Society of the Pacific, San Francisco (Cited on page 43.)

Aschwanden, M.J., 2009b, "4-D modeling of CME expansion and EUV dimming observed with STEREO/EUVI", Ann. Geophys., 27, 3275-3286. [DOI], [ADS], [arXiv:0908.1913 [astro-ph.SR]] (Cited on page 68.)

Aschwanden, M.J., 2009c, "The 3D Geometry, Motion, and Hydrodynamic Aspects of Oscillating Coronal Loops", Space Sci. Rev., 149, 31-64. [DOI], [ADS] (Cited on pages 22, 48, 49, and 53.)

Aschwanden, M.J., 2010, "A Code for Automated Tracing of Coronal Loops Approaching Visual Perception", Solar Phys., 262, 399-423. [DOI], [ADS] (Cited on page 38.)

Living Reviews in Solar Physics

http://www. livingreviews.org/lrsp-2011-5 
Aschwanden, M.J., 2011, Self-Organized Criticality in Astrophysics: The Statistics of Nonlinear Processes in the Universe, Springer; Praxis, Berlin; New York; Chichester. [ADS], [Google Books] (Cited on page 66.)

Aschwanden, M.J. and Acton, L.W., 2001, "Temperature Tomography of the Soft X-Ray Corona: Measurements of Electron Densities, Tempuratures, and Differential Emission Measure Distributions above the Limb", Astrophys. J., 550, 475-492. [DOI], [ADS] (Cited on page 29.)

Aschwanden, M.J. and Bastian, T.S., 1994a, "VLA stereoscopy of solar active regions. I. Method and tests", Astrophys. J., 426, 425-433. [DOI], [ADS] (Cited on pages 6, 9, and 32.)

Aschwanden, M.J. and Bastian, T.S., 1994b, "VLA stereoscopy of solar active regions. II. Altitude, relative motion, and center-to-limb darkening of 20 centimeter emission", Astrophys. J., 426, 434-448. [DOI], [ADS] (Cited on pages 6, 9, 10, 32, and 33.)

Aschwanden, M.J. and Boerner, P., 2011, "Solar Corona Loop Studies with AIA: I. Cross-Sectional Temperature Structure", Astrophys. J., 732, 81. [DOI], [ADS], [arXiv:1103.0228 [astro-ph.SR]] (Cited on page 35.)

Aschwanden, M.J. and Sandman, A.W., 2010, "Bootstrapping the Coronal Magnetic Field with STEREO: Unipolar Potential Field Modeling", Astron. J., 140, 723-734. [DOI], [ADS], [arXiv:1004.4920 [astro-ph.SR]] (Cited on pages 22, 25, 45, 46, and 47.)

Aschwanden, M.J. and Tsiklauri, D., 2009, "The Hydrodynamic Evolution of Impulsively Heated Coronal Loops: Explicit Analytical Approximations", Astrophys. J. Suppl. Ser., 185, 171-185. [DOI], [ADS] (Cited on page 40.)

Aschwanden, M.J. and Wülser, J.-P., 2011, "3-D reconstruction of active regions with STEREO", J. Atmos. Sol.-Terr. Phys., 73, 1082-1095. [DOI] (Cited on pages 5 and 33.)

Aschwanden, M.J., Bastian, T.S. and White, S.M., 1992, "3D reconstruction methods of coronal structures by radio observations", in Coronal Streamers, Coronal Loops, and Coronal and Solar Wind Composition, Proceedings of the First SOHO Workshop, held in Annapolis, MD, 25-28 August 1992, (Ed.) Mattok, C., vol. 348 of ESA Conference Proceedings, pp. 217-220, ESA Publications Division, Noordwijk. [ADS] (Cited on pages 6, 9, and 17.)

Aschwanden, M.J., Lim, J., Gary, D.E. and Klimchuk, J.A., 1995, "Solar Rotation Stereoscopy in Microwaves", Astrophys. J., 454, 512-521. [DOI], [ADS] (Cited on pages 6, 11, and 22.)

Aschwanden, M.J., Newmark, J.S., Delaboudinière, J.-P., Neupert, W.M., Klimchuk, J.A., Gary, G.A., Portier-Fozzani, F. and Zucker, A., 1999, "Three-dimensional Stereoscopic Analysis of Solar Active Region Loops. I. SOHO/EIT Observations at Temperatures of $(1.0-1.5) \times 10^{6}$ K", Astrophys. J., 515, 842-867. [DOI], [ADS] (Cited on pages 7, 11, 12, 33, 38, and 40.)

Aschwanden, M.J., Alexander, D., Hurlburt, N., Newmark, J.S., Neupert, W.M., Klimchuk, J.A. and Gary, G.A., 2000, "Three-dimensional Stereoscopic Analysis of Solar Active Region Loops. II. SOHO/EIT Observations at Temperatures of 1.5-2.5 MK", Astrophys. J., 531, 1129-1149. [DOI], [ADS] (Cited on pages $7,11,33,38,40$, and 43.)

Aschwanden, M.J., Alexander, D. and DeRosa, M.L., 2004, "Tomographic 3D-Modeling of the Solar Corona with FASR", in Solar and Space Weather Radiophysics: Current Status and Future Developments, (Eds.) Gary, D.E., Keller, C.U., vol. 314 of Astrophysics and Space Science Library, pp. 243-264, Kluwer Academic Publishers, Dordrecht. [Google Books] (Cited on page 17.) 
Aschwanden, M.J., Burlaga, L.F., Kaiser, M.L., Ng, C.K., Reames, D.V., Reiner, M.J., Gombosi, T.I., Lugaz, N., Manchester IV, W.B., Roussev, I.I., Zurbuchen, T.H., Farrugia, C.J., Galvin, A.B., Lee, M.A., Linker, J.A., Mikić, Z., Riley, P., Alexander, D., Sandman, A.W., Cook, J.W., Howard, R.A., Odstrčil, D., Pizzo, V.J., Kóta, J., Liewer, P.C., Luhmann, J.G., Inhester, B., Schwenn, R.W., Solanki, S.K., Vasyliunas, V.M., Wiegelmann, T., Blush, L., Bochsler, P., Cairns, I.H., Robinson, P.A., Bothmer, V., Kecskemety, K., Llebaria, A., Maksimovic, M., Scholer, M. and Wimmer-Schweingruber, R.F., 2008a, "Theoretical modeling for the STEREO mission", Space Sci. Rev., 136, 565-604. [DOI], [ADS] (Cited on page 5.)

Aschwanden, M.J., Nitta, N.V., Wülser, J.-P. and Lemen, J.R., 2008b, "First 3D Reconstructions of Coronal Loops with the STEREO A+B Spacecraft. II. Electron Density and Temperature Measurements", Astrophys. J., 680, 1477-1495. [DOI], [ADS] (Cited on pages 21, 33, 40, 42, and 43.)

Aschwanden, M.J., Wülser, J.-P., Nitta, N.V. and Lemen, J.R., 2008c, "First Three-Dimensional Reconstructions of Coronal Loops with the STEREO A and B Spacecraft. I. Geometry", Astrophys. J., 679, 827-842. [DOI], [ADS] (Cited on pages 22, 33, and 38.)

Aschwanden, M.J., Nitta, N.V., Wülser, J.-P., Lemen, J.R., Sandman, A., Vourlidas, A. and Colaninno, R.C., 2009a, "First Measurements of the Mass of Coronal Mass Ejections from the EUV Dimming Observed with STEREO EUVI A+B Spacecraft", Astrophys. J., 706, 376-392. [DOI], [ADS] (Cited on page 69.)

Aschwanden, M.J., Wülser, J.-P., Nitta, N.V. and Lemen, J.R., 2009b, "Solar Flare and CME Observations with STEREO/EUVI", Solar Phys., 256, 3-40. [DOI], [ADS] (Cited on pages 48, $51,59,65,68,69$, and 70.$)$

Aschwanden, M.J., Wülser, J.-P., Nitta, N.V., Lemen, J.R. and Sandman, A., 2009c, "First ThreeDimensional Reconstructions of Coronal Loops with the STEREO A+B Spacecraft. III. Instant Stereoscopic Tomography of Active Regions", Astrophys. J., 695, 12-29. [DOI], [ADS] (Cited on pages $25,26,33,34,35,36$, and 39 .)

Aschwanden, M.J., Boerner, P., Schrijver, C.J. and Malanushenko, A., 2011, "Automated temperature and emission measure analysis of coronal loops and active regions observed with AIA/SDO", The First LWS/SDO Workshop: The Many Spectra of Solar Activity, Squaw Creek, CA, May $1-5,2011$, conference paper (Cited on page 35.)

Attrill, G.D.R. and Wills-Davey, M.J., 2010, "Automatic Detection and Extraction of Coronal Dimmings from SDO/AIA Data", Solar Phys., 262, 461-480. [DOI], [ADS] (Cited on page 71.)

Attrill, G.D.R., Harra, L.K., van Driel-Gesztelyi, L. and Démoulin, P., 2007, "Coronal 'Wave': Magnetic Footprint of a Coronal Mass Ejection?", Astrophys. J. Lett., 656, L101-L104. [DOI], [ADS] (Cited on page 71.)

Attrill, G.D.R., Engell, A.J., Wills-Davey, M.J., Grigis, P. and Testa, P., 2009, "Hinode/XRT and STEREO Observations of a Diffuse Coronal 'Wave'-Coronal Mass Ejection-Dimming Event", Astrophys. J., 704, 1296-1308. [DOI], [ADS] (Cited on page 72.)

Barbey, N., Auchère, F., Rodet, T. and Vial, J.-C., 2008, "A Time-Evolving 3D Method Dedicated to the Reconstruction of Solar Plumes and Results Using Extreme Ultraviolet Data", Solar Phys., 248, 409-423. [DOI], [ADS], [arXiv:0802.0113] (Cited on pages 26 and 62.)

Barbey, N., Guennou, C. and Auchère, F., 2011, "TomograPy: A fast, instrument-independent, solar tomography software", arXiv, e-print. [ADS], [arXiv:1103.5904] (Cited on page 15.) 
Batchelor, D., 1994, "Quasi-stereoscopic imaging of the solar X-ray corona", Solar Phys., 155, 57-61. [DOI], [ADS] (Cited on page 7.)

Bemporad, A., 2009, "Stereoscopic Reconstruction from STEREO/EUV Imagers Data of the Three-dimensional Shape and Expansion of an Erupting Prominence", Astrophys. J., 701, 298305. [DOI], [ADS] (Cited on pages 22 and 59.)

Bemporad, A., Del Zanna, G., Andretta, V., Poletto, G. and Magrí, M., 2009, "Multispacecraft observations of a prominence eruption", Ann. Geophys., 27, 3841-3851. [DOI], [ADS] (Cited on page 59.)

Berton, R. and Sakurai, T., 1985, "Stereoscopic determination of the three-dimensional geometry of coronal magnetic loops", Solar Phys., 96, 93-111. [DOI], [ADS] (Cited on pages 7, 9, and 38.)

Bewsher, D., Harrison, R.A. and Brown, D.S., 2008, "The relationship between EUV dimming and coronal mass ejections. I. Statistical study and probability model", Astron. Astrophys., 478, 897-906. [DOI], [ADS] (Cited on page 67.)

Billings, D.E., 1966, A Guide to the Solar Corona, Academic Press, New York; London. [ADS] (Cited on page 14.)

Bogod, V.M. and Grebinskij, A.S., 1997, "Large-Scale Structure of the Atmosphere of the Quiet Sun, Coronal Holes, and Plages as Deduced by Tomography Study", Solar Phys., 176, 67-86. [DOI], [ADS] (Cited on pages 6 and 17.)

Bone, L.A., van Driel-Gesztelyi, L., Culhane, J.L., Aulanier, G. and Liewer, P., 2009, "Formation, Interaction and Merger of an Active Region and a Quiescent Filament Prior to Their Eruption on 19 May 2007", Solar Phys., 259, 31-47. [DOI], [ADS] (Cited on page 59.)

Bothmer, V., Bougeret, J.-L., Cargill, P., Davila, J., Delaboudiniere, J.-P., Harrison, R., Koutchmy, S., Liewer, P., Maltby, P., Rust, D. and Schwenn, R., 1998, "European Plans for the Solar STEREO Mission - brief summary of the European STEREO steering team (ESST) -", in A Crossroads for European Solar and Heliospheric Physics: Recent Achievements and Future Mission Possibilities, Proceedings of a conference held at Puerto de la Cruz, Tenerife, Canary Islands, Spain, March 23-27, 1998, (Eds.) Priest, E.R., Moreno-Insertis, F., Harris, R.A., vol. 417 of ESA Conference Proceedings, pp. 145-151, ESA Publications Division, Noordwijk. [ADS] (Cited on page 8.)

Bougeret, J.L., Goetz, K., Kaiser, M.L., Bale, S.D., Kellogg, P.J., Maksimovic, M., Monge, N., Monson, S.J., Astier, P.L., Davy, S., Dekkali, M., Hinze, J.J., Manning, R.E., Aguilar-Rodriguez, E., Bonnin, X., Briand, C., Cairns, I.H., Cattell, C.A., Cecconi, B., Eastwood, J., Ergun, R.E., Fainberg, J., Hoang, S., Huttunen, K.E.J., Krucker, S., Lecacheux, A., MacDowall, R.J., Macher, W., Mangeney, A., Meetre, C.A., Moussas, X., Nguyen, Q.N., Oswald, T.H., Pulupa, M., Reiner, M.J., Robinson, P.A., Rucker, H., Salem, C., Santolik, O., Silvis, J.M., Ullrich, R., Zarka, P. and Zouganelis, I., 2008, "S/WAVES: The Radio and Plasma Wave Investigation on the STEREO Mission", Space Sci. Rev., 136, 487-528. [DOI], [ADS] (Cited on page 8.)

Brosius, J.W., Davila, J.M., Thomas, R.J. and Monsignori-Fossi, B.C., 1996, "Measuring Active and Quiet-Sun Coronal Plasma Properties with Extreme-Ultraviolet Spectra from SERTS", Astrophys. J. Suppl. Ser., 106, 143-164. [DOI], [ADS] (Cited on page 35.)

Brown, D.S., 2009, "Our explosive sun", Phys. Educ., 44, 20-26. [DOI], [ADS] (Cited on page 5.) 
Butala, M.D., Frazin, R.A. and Kamalabadi, F., 2005, "Three-dimensional estimates of the coronal electron density at times of extreme solar activity", J. Geophys. Res., 110, A09S09. [DOI], [ADS] (Cited on page 15.)

Butala, M.D., Hewett, R.J., Frazin, R.A. and Kamalabadi, F., 2010, "Dynamic Three-Dimensional Tomography of the Solar Corona", Solar Phys., 262, 495-509. [DOI], [ADS] (Cited on page 29.)

Caroubalos, C. and Steinberg, J.L., 1974, "Evidence of Solar Burst Directivity at $169 \mathrm{MHz}$ from Simultaneous Ground Based and Deep Space Observations (STEREO-1 Preliminary Results)", Astron. Astrophys., 32, 245-253. [ADS] (Cited on page 7.)

Chen, J. and Kunkel, V., 2010, "Temporal and Physical Connection Between Coronal Mass Ejections and Flares", Astrophys. J., 717, 1105-1122. [DOI], [ADS] (Cited on page 66.)

Chen, P.F., 2011, "Coronal Mass Ejections: Models and Their Observational Basis", Living Rev. Solar Phys., 8, lrsp-2011-1. [ADS]. URL (accessed 9 May 2011): http://www.livingreviews.org/lrsp-2011-1 (Cited on pages 67 and 71.)

Cheng, X., Ding, M.D. and Zhang, J., 2010, "A Study of the Build-up, Initiation, and Acceleration of 2008 April 26 Coronal Mass Ejection Observed by STEREO", Astrophys. J., 712, 1302-1310. [DOI], [ADS], [arXiv:1002.4962 [astro-ph.SR]] (Cited on page 67.)

Chifor, C., Isobe, H., Mason, H.E., Hannah, I.G., Young, P.R., Del Zanna, G., Krucker, S., Ichimoto, K., Katsukawa, Y. and Yokoyama, T., 2008, "Magnetic flux cancellation associated with a recurring solar jet observed with Hinode, RHESSI, and STEREO/EUVI", Astron. Astrophys., 491, 279-288. [DOI], [ADS] (Cited on page 62.)

Cohen, O., Attrill, G.D.R., Manchester IV, W.B. and Wills-Davey, M.J., 2009, "Numerical Simulation of an EUV Coronal Wave Based on the 2009 February 13 CME Event Observed by STEREO", Astrophys. J., 705, 587-602. [DOI], [ADS], [arXiv:0909.3095 [astro-ph.SR]] (Cited on pages 71 and 72 .)

Colaninno, R.C. and Vourlidas, A., 2009, "First Determination of the True Mass of Coronal Mass Ejections: A Novel Approach to Using the Two STEREO Viewpoints", Astrophys. J., 698, 852-858. [DOI], [ADS], [arXiv:0903.4344 [astro-ph.SR]] (Cited on page 69.)

Conlon, P.A. and Gallagher, P.T., 2010, "Constraining Three-Dimensional Magnetic Field Extrapolations Using the Twin Perspectives of STEREO", Astrophys. J., 715, 59-65. [DOI], [ADS], [arXiv:1004.1330 [astro-ph.SR]] (Cited on pages 22 and 45.)

Culhane, J.L., Bone, L., Hara, H., Farrugia, C., Galvin, A., van Driel-Gesztelyi, L., Popecki, M., Luhmann, J.G. and Veronig, A., 2008, "Flare and Erupting Filament of 19th May, 2007 Sources of a Magnetic Cloud Observed by Stereo", 37th COSPAR Scientific Assembly, Montréal, Canada, 13 - 20 July 2008, conference paper. [ADS] (Cited on page 59.)

Curdt, W., Wilhelm, K., Feng, L. and Kamio, S., 2008, "Multi-spacecraft observations of polar coronal plumes", Astron. Astrophys., 481, L61-L64. [DOI], [ADS] (Cited on page 62.)

Davila, J.M., 1994, "Solar Tomography", Astrophys. J., 423, 871-877. [DOI], [ADS] (Cited on pages 25 and 26.)

Davila, J.M., 1996, "Tomography of the solar corona and the inner heliosphere: the solar imaging of coronal extended structures (SLICES)", in Missions to the Sun, Denver, CO, USA, 8 August 1996, (Ed.) Rust, D.M., vol. 2804 of Proc. SPIE, pp. 62-68, SPIE, Bellingham, WA. [DOI], [ADS] (Cited on page 25.) 
Davila, J.M., 1998, "Observing the inner heliosphere from new perspectives", Adv. Space Res., 21, 319-323. [DOI], [ADS] (Cited on pages 5, 8, and 25.)

Davila, J.M. and Thompson, W.T., 1992, "A rotating tomographic imager for solar extremeultraviolet/soft X-ray emission", Astrophys. J. Lett., 389, L91-L93. [DOI], [ADS] (Cited on pages 14 and 25.)

Davila, J.M., Rust, D.M., Pizzo, V.J. and Liewer, P.C., 1996, "Solar Terrestrial Relations Observatory (STEREO)", in Missions to the Sun, Denver, CO, USA, 8 August 1996, (Ed.) Rust, D.M., vol. 2804 of Proc. SPIE, pp. 34-38, SPIE, Bellingham, WA. [DOI], [ADS] (Cited on page 8.)

Delouille, V., Chainais, P. and Hochedez, J.-F., 2008, "Spatial and Temporal Noise in Solar EUV Observations", Solar Phys., 248, 441-455. [DOI], [ADS] (Cited on page 8.)

Démoulin, P. and Aulanier, G., 2010, "Criteria for Flux Rope Eruption: Non-equilibrium Versus Torus Instability", Astrophys. J., 718, 1388-1399. [DOI], [ADS], [arXiv:1006.1785 [astro-ph.SR]] (Cited on page 67.)

Dere, K.P., Wang, D. and Howard, R.A., 2005, "Three-dimensional Structure of Coronal Mass Ejections from LASCO Polarization Measurements", Astrophys. J. Lett., 620, L119-L122. [DOI], [ADS] (Cited on page 7.)

DeRosa, M.L., Schrijver, C.J., Barnes, G., Leka, K.D., Lites, B.W., Aschwanden, M.J., Amari, T., Canou, A., McTiernan, J.M., Régnier, S., Thalmann, J.K., Valori, G., Wheatland, M.S., Wiegelmann, T., Cheung, M.C.M., Conlon, P.A., Fuhrmann, M., Inhester, B. and Tadesse, T., 2009, "A Critical Assessment of Nonlinear Force-Free Field Modeling of the Solar Corona for Active Region 10953", Astrophys. J., 696, 1780-1791. [DOI], [ADS], [arXiv:0902.1007 [astro-ph.SR]] (Cited on pages 22, 24, 44, and 46.)

Downs, C., Roussev, I.I., van der Holst, B., Lugaz, N., Sokolov, I.V. and Gombosi, T.I., 2011, "Studying Extreme Ultraviolet Wave Transients with a Digital Laboratory: Direct Comparison of Extreme Ultraviolet Wave Observations to Global Magnetohydrodynamic Simulations", Astrophys. J., 728, 2. [DOI], [ADS] (Cited on page 72.)

Driesman, A., Hynes, S. and Cancro, G., 2008, "The STEREO Observatory", Space Sci. Rev., 136, 17-44. [DOI], [ADS] (Cited on page 8.)

Feng, L., Inhester, B., Solanki, S.K., Wiegelmann, T., Podlipnik, B., Howard, R.A. and Wülser, J.P., 2007a, "First Stereoscopic Coronal Loop Reconstructions from STEREO SECCHI Images", Astrophys. J. Lett., 671, L205-L208. [DOI], [ADS], [arXiv:0802.0773] (Cited on pages 22, 38, and 44.)

Feng, L., Wiegelmann, T., Inhester, B., Solanki, S., Gan, W.Q. and Ruan, P., 2007b, "Magnetic Stereoscopy of Coronal Loops in NOAA 8891", Solar Phys., 241, 235-249. [DOI], [ADS] (Cited on pages 22 and 44.)

Feng, L., Inhester, B., Solanki, S.K., Wilhelm, K., Wiegelmann, T., Podlipnik, B., Howard, R.A., Plunkett, S.P., Wülser, J.-P. and Gan, W.Q., 2009, "Stereoscopic Polar Plume Reconstructions from STEREO/SECCHI Images", Astrophys. J., 700, 292-301. [DOI], [ADS], [arXiv:0908.2365 [astro-ph.SR]] (Cited on pages 22 and 62.)

Forbes, T.G., Linker, J.A., Chen, J., Cid, C., Kóta, J., Lee, M.A., Mann, G., Mikić, Z., Potgieter, M.S., Schmidt, J.M., Siscoe, G.L., Vainio, R., Antiochos, S.K. and Riley, P., 2006, "CME Theory and Models", Space Sci. Rev., 123, 251-302. [DOI], [ADS] (Cited on page 67.) 
Foullon, C., 2003, "Automated detection and 3D reconstruction of EUV prominences", in Solar Variability as an Input to the Earth's Environment, International Solar Cycle Studies (ISCS) Symposium held in Tatranská Lomnica, Slovak Republic, 23-28 June 2003, (Ed.) Wilson, A., vol. 535 of ESA Conference Proceedings, pp. 477-482, ESA Publications Division, Noordwijk. [ADS] (Cited on pages 7 and 58.)

Frazin, R.A., 2000, "Tomography of the Solar Corona. I. A Robust, Regularized, Positive Estimation Method", Astrophys. J., 530, 1026-1035. [DOI], [ADS] (Cited on pages 13 and 15.)

Frazin, R.A. and Janzen, P., 2002, "Tomography of the Solar Corona. II. Robust, Regularized, Positive Estimation of the Three-dimensional Electron Density Distribution from LASCO-C2 Polarized White-Light Images", Astrophys. J., 570, 408-422. [DOI], [ADS] (Cited on pages 13 and 15.)

Frazin, R.A. and Kamalabadi, F., 2005a, "On the Use of Total Brightness Measurements for Tomography of the Solar Corona", Astrophys. J., 628, 1061-1069. [DOI], [ADS] (Cited on page 15.)

Frazin, R.A. and Kamalabadi, F., 2005b, "Rotational Tomography for 3D Reconstruction of the White-Light and EUV Corona in the Post-SOHO Era", Solar Phys., 228, 219-237. [DOI], [ADS] (Cited on pages 13 and 15.)

Frazin, R.A., Butala, M.D., Kemball, A. and Kamalabadi, F., 2005a, "Time-dependent Reconstruction of Nonstationary Objects with Tomographic or Interferometric Measurements", Astrophys. J. Lett., 635, L197-L200. [DOI], [ADS], [arXiv:astro-ph/0509472] (Cited on pages 13 and 29.)

Frazin, R.A., Kamalabadi, F. and Weber, M.A., 2005b, "On the Combination of Differential Emission Measure Analysis and Rotational Tomography for Three-dimensional Solar EUV Imaging", Astrophys. J., 628, 1070-1080. [DOI], [ADS] (Cited on page 13.)

Frazin, R.A., Vásquez, A.M., Kamalabadi, F. and Park, H., 2007, "Three-dimensional Tomographic Analysis of a High-Cadence LASCO-C2 Polarized Brightness Sequence", Astrophys. J. Lett., 671, L201-L204. [DOI], [ADS] (Cited on pages 7, 28, and 31.)

Frazin, R.A., Jacob, M., Manchester IV, W.B., Morgan, H. and Wakin, M.B., 2009a, "Toward Reconstruction of Coronal Mass Ejection Density from Only Three Points of View", Astrophys. J., 695, 636-641. [DOI], [ADS] (Cited on page 26.)

Frazin, R.A., Vásquez, A.M. and Kamalabadi, F., 2009b, "Quantitative, Three-dimensional Analysis of the Global Corona with Multi-spacecraft Differential Emission Measure Tomography", Astrophys. J., 701, 547-560. [DOI], [ADS] (Cited on pages 13, 28, and 29.)

Frazin, R.A., Lamy, P., Llebaria, A. and Vásquez, A.M., 2010, "Three-Dimensional Electron Density from Tomographic Analysis of LASCO-C2 Images of the K-Corona Total Brightness", Solar Phys., 265, 19-30. [DOI], [ADS] (Cited on pages 28 and 29.)

Galvin, A.B., Kistler, L.M., Popecki, M.A., Farrugia, C.J., Simunac, K.D.C., Ellis, L., Möbius, E., Lee, M.A., Boehm, M., Carroll, J., Crawshaw, A., Conti, M., Demaine, P., Ellis, S., Gaidos, J.A., Googins, J., Granoff, M., Gustafson, A., Heirtzler, D., King, B., Knauss, U., Levasseur, J., Longworth, S., Singer, K., Turco, S., Vachon, P., Vosbury, M., Widholm, M., Blush, L.M., Karrer, R., Bochsler, P., Daoudi, H., Etter, A., Fischer, J., Jost, J., Opitz, A., Sigrist, M., Wurz, P., Klecker, B., Ertl, M., Seidenschwang, E., Wimmer-Schweingruber, R.F., Koeten, M., Thompson, B. and Steinfeld, D., 2008, "The Plasma and Suprathermal Ion Composition (PLASTIC) Investigation on the STEREO Observatories", Space Sci. Rev., 136, 437-486. [DOI], [ADS] (Cited on page 8.) 
Gary, G.A., 1997, "Rendering Three-Dimensional Solar Coronal Structures", Solar Phys., 174, 241-263. [DOI], [ADS] (Cited on pages 26 and 27.)

Gary, G.A. and Alexander, D., 1999, "Constructing the Coronal Magnetic Field By Correlating Parameterized Magnetic Field Lines With Observed Coronal Plasma Structures", Solar Phys., 186, 123-139. [DOI], [ADS] (Cited on page 40.)

Gary, G.A., Davis, J.M. and Moore, R., 1998, "On Analysis of Dual Spacecraft Stereoscopic Observations to Determine the Three-Dimensional Morphology and Plasma Properties of Solar Coronal Flux Tubes", Solar Phys., 183, 45-76. [DOI], [ADS] (Cited on page 40.)

Gelfreikh, G.B., 1998, "Three-dimensional Structure of the Magnetospheres of Solar Active Regions from Radio Observations (Invited review)", in Three-Dimensional Structure of Solar Active Regions, 2nd Advances in Solar Physics Euroconference, (Eds.) Alissandrakis, C.E., Schmieder, B., vol. 155 of ASP Conference Series, pp. 110-129, Astronomical Society of the Pacific, San Francisco. [ADS] (Cited on pages 6 and 17.)

Gibson, S.E., Kucera, T.A., Rastawicki, D., Dove, J., de Toma, G., Hao, J., Hill, S., Hudson, H.S., Marqué, C., McIntosh, P.S., Rachmeler, L., Reeves, K.K., Schmieder, B., Schmit, D.J., Seaton, D.B., Sterling, A.C., Tripathi, D., Williams, D.R. and Zhang, M., 2010, "Three-dimensional Morphology of a Coronal Prominence Cavity", Astrophys. J., 724, 1133-1146. [DOI], [ADS] (Cited on page 59.)

Gissot, S.F., Hochedez, J.-F., Chainais, P. and Antoine, J.-P., 2008, "3D Reconstruction from SECCHI-EUVI Images Using an Optical-Flow Algorithm: Method Description and Observation of an Erupting Filament", Solar Phys., 252, 397-408. [DOI], [ADS] (Cited on pages 26 and 59.)

Gopalswamy, N., Yashiro, S., Temmer, M., Davila, J., Thompson, W.T., Jones, S., McAteer, R.T.J., Wülser, J.-P., Freeland, S. and Howard, R.A., 2009, "EUV Wave Reflection from a Coronal Hole", Astrophys. J. Lett., 691, L123-L127. [DOI], [ADS] (Cited on page 72.)

Gosain, S. and Schmieder, B., 2010, "Estimation of width and inclination of a filament sheet using He II $304 \AA$ Å observations by STEREO/EUVI", Ann. Geophys., 28, 149-153. [DOI], [ADS], [arXiv:1001.3004 [astro-ph.SR]] (Cited on page 58.)

Gosain, S., Schmieder, B., Venkatakrishnan, P., Chandra, R. and Artzner, G.E., 2009, "3D Evolution of a Filament Disappearance Event Observed by STEREO", Solar Phys., 259, 13-30. [DOI], [ADS], [arXiv:0910.0786 [astro-ph.SR]] (Cited on page 59.)

Grebinskij, A., Bogod, V., Gelfreikh, G., Urpo, S., Pohjolainen, S. and Shibasaki, K., 2000, "Microwave tomography of solar magnetic fields", Astron. Astrophys. Suppl., 144, 169-180. [DOI], [ADS] (Cited on pages 6 and 17.)

Grigoryev, V.M., 1993, "A space-borne solar stereoscope experiment in solar physics", Solar Phys., 148, 389-391. [DOI], [ADS] (Cited on page 8.)

Harrison, R.A., Davies, J.A., Rouillard, A.P., Davis, C.J., Eyles, C.J., Bewsher, D., Crothers, S.R., Howard, R.A., Sheeley, N.R., Vourlidas, A., Webb, D.F., Brown, D.S. and Dorrian, G.D., 2009, "Two Years of the STEREO Heliospheric Imagers: Invited Review", Solar Phys., 256, 219-237. [DOI], [ADS] (Cited on pages 5 and 8.)

He, J.-S., Marsch, E., Curdt, W., Tian, H., Tu, C.-Y., Xia, L.-D. and Kamio, S., 2010, "Magnetic and spectroscopic properties of supergranular-scale coronal jets and erupting loops in a polar coronal hole", Astron. Astrophys., 519, A49. [DOI], [ADS] (Cited on page 62.) 
Howard, R.A., Moses, J.D., Socker, D.G., Dere, K.P. and Cook, J.W., 2002, "Sun earth connection coronal and heliospheric investigation (SECCHI)", Adv. Space Res., 29, 2017-2026. [DOI], [ADS] (Cited on page 8.)

Howard, R.A., Moses, J.D., Vourlidas, A., Newmark, J.S., Socker, D.G., Plunkett, S.P., Korendyke, C.M., Cook, J.W., Hurley, A., Davila, J.M., Thompson, W.T., St Cyr, O.C., Mentzell, E., Mehalick, K., Lemen, J.R., Wülser, J.-P., Duncan, D.W., Tarbell, T.D., Wolfson, C.J., Moore, A., Harrison, R.A., Waltham, N.R., Lang, J., Davis, C.J., Eyles, C.J., Mapson-Menard, H., Simnett, G.M., Halain, J.P., Defise, J.M., Mazy, E., Rochus, P., Mercier, R., Ravet, M.-F., Delmotte, F., Auchere, F., Delaboudinière, J.P., Bothmer, V., Deutsch, W., Wang, D., Rich, N., Cooper, S., Stephens, V., Maahs, G., Baugh, R., McMullin, D. and Carter, T., 2008, "Sun Earth Connection Coronal and Heliospheric Investigation (SECCHI)", Space Sci. Rev., 136, 67-115. [DOI], [ADS] (Cited on page 8.)

Howard, T.A. and Tappin, S.J., 2009a, "Interplanetary Coronal Mass Ejections Observed in the Heliosphere: 1. Review of Theory", Space Sci. Rev., 147, 31-54. [DOI], [ADS] (Cited on pages 5 and 14.)

Howard, T.A. and Tappin, S.J., 2009b, "Interplanetary Coronal Mass Ejections Observed in the Heliosphere: 3. Physical Implications", Space Sci. Rev., 147, 89-110. [DOI], [ADS] (Cited on page 5.)

Hurlburt, N.E., Martens, P.C.H., Slater, G.L. and Jaffey, S.M., 1994, "Volume Reconstruction of Magnetic Fields using Solar Imagery", in Solar Active Region Evolution: Comparing Models with Observations, Proceedings of the 14th International Summer Workshop, NSO / Sac Peak, Sunspot, New Mexico, USA, 30 August-3 September 1993, (Eds.) Balasubramaniam, K.S., Simon, G.W., vol. 68 of ASP Conference Series, pp. 30-36, Astronomical Society of the Pacific, San Francisco. [ADS] (Cited on pages 7 and 13.)

Inhester, B., 2006, "Stereoscopy basics for the STEREO mission", e-print. [ADS], [astro-ph/0612649] (Cited on pages 18, 19, and 20.)

Inhester, B., Feng, L. and Wiegelmann, T., 2008, "Segmentation of Loops from Coronal EUV Images", Solar Phys., 248, 379-393. [DOI], [ADS], [arXiv:0801.3240] (Cited on page 38.)

Innes, D.E., McIntosh, S.W. and Pietarila, A., 2010, "STEREO quadrature observations of coronal dimming at the onset of mini-CMEs", Astron. Astrophys., 517, L7. [DOI], [ADS], [arXiv:1005.2097 [astro-ph.SR]] (Cited on page 71.)

Jackson, B.V. and Froehling, H.R., 1995, "Three-dimensional reconstruction of a coronal mass ejection", Astron. Astrophys., 299, 885-892. [ADS] (Cited on page 6.)

Jackson, B.V. and Hick, P.P., 2002, "Corotational Tomography of Heliospheric Features Using Global Thomson Scattering Data", Solar Phys., 211, 345-356. [DOI], [ADS] (Cited on page 7.)

Jackson, B.V. and Hick, P.P., 2004, "Three-dimensional tomography of interplanetary disturbances", in Solar and Space Weather Radiophysics: Current Status and Future Developments, (Eds.) Gary, D.E., Keller, C.U., vol. 314 of Astrophysics and Space Science Library, pp. 355-386, Kluwer Academic Publishers, Dordrecht. [Google Books] (Cited on page 7.)

Jackson, B.V., Buffington, A., Hick, P.P., Altrock, R.C., Figueroa, S., Holladay, P.E., Johnston, J.C., Kahler, S.W., Mozer, J.B., Price, S., Radick, R.R., Sagalyn, R., Sinclair, D., Simnett, G.M., Eyles, C.J., Cooke, M.P., Tappin, S.J., Kuchar, T., Mizuno, D., Webb, D.F., Anderson, P.A., Keil, S.L., Gold, R.E. and Waltham, N.R., 2004, "The Solar Mass-Ejection Imager (SMEI) Mission", Solar Phys., 225, 177-207. [DOI], [ADS] (Cited on page 7.)

Living Reviews in Solar Physics

http://www. livingreviews . org/lrsp-2011-5 
Jackson, J.D., 1962, Classical Electrodynamics, John Wiley \& Sons, New York (Cited on page 14.)

Jing, J., Tan, C., Yuan, Y., Wang, B., Wiegelmann, T., Xu, Y. and Wang, H., 2010, "Free Magnetic Energy and Flare Productivity of Active Regions", Astrophys. J., 713, 440-449. [DOI], [ADS] (Cited on page 45.)

Kaiser, M.L., 2005, "The STEREO mission: an overview", Adv. Space Res., 36, 1483-1488. [DOI], [ADS] (Cited on pages 5 and 8.)

Kaiser, M.L., Kucera, T.A., Davila, J.M., St Cyr, O.C., Guhathakurta, M. and Christian, E., 2008, "The STEREO Mission: An Introduction", Space Sci. Rev., 136, 5-16. [DOI], [ADS] (Cited on pages 5 and 8 .)

Kamio, S., Curdt, W., Teriaca, L., Inhester, B. and Solanki, S.K., 2010, "Observations of a rotating macrospicule associated with an X-ray jet", Astron. Astrophys., 510, L1. [DOI], [ADS], [arXiv:1001.1924 [astro-ph.SR]] (Cited on page 62.)

Kane, S.R., 1981, "Multi-spacecraft observations of solar hard X-ray bursts", Astrophys. Space Sci., 75, 163-179. [DOI], [ADS] (Cited on page 7.)

Kane, S.R., McTiernan, J., Loran, J., Fenimore, E.E., Klebesadel, R.W. and Laros, J.G., 1992, "Stereoscopic observations of a solar flare hard X-ray source in the high corona", Astrophys. J., 390, 687-702. [DOI], [ADS] (Cited on page 7.)

Kane, S.R., Hurley, K., McTiernan, J.M., Boer, M., Niel, M., Kosugi, T. and Yoshimori, M., 1998, "Stereoscopic Observations of Solar Hard X-Ray Flares Made by ULYSSES and YOHKOH", Astrophys. J., 500, 1003-1008. [DOI], [ADS] (Cited on page 7.)

Khodachenko, M.L. and Rucker, H.O., 2005, "Solar plasma theoretical models for STEREO and Solar-B", Adv. Space Res., 36, 1561-1571. [DOI], [ADS] (Cited on page 5.)

Kienreich, I.W., Temmer, M. and Veronig, A.M., 2009, "STEREO Quadrature Observations of the Three-Dimensional Structure and Driver of a Global Coronal Wave", Astrophys. J. Lett., 703, L118-L122. [DOI], [ADS], [arXiv:0908.3571 [astro-ph.SR]] (Cited on pages 71 and 72.)

Kontar, E.P. and Brown, J.C., 2006, "Stereoscopic Electron Spectroscopy of Solar Hard X-Ray Flares with a Single Spacecraft", Astrophys. J. Lett., 653, L149-L152. [DOI], [ADS], [arXiv:astroph/0611170] (Cited on page 65.)

Koutchmy, S. and Molodenskii, M.M., 1992, "Three-dimensional image of the solar corona from white-light observations of the 1991 eclipse", Nature, 360, 717-719. [DOI], [ADS] (Cited on page 6.)

Koutchmy, S., Molodenskii, M.M., Starkova, L.I., Kutvitskii, V.A., Ershov, A.V., Ivanov, A.A., Petrov, B.B. and Sidorov, C.C., 1997, "The heliospheric layer and the coronal structure on November 3, 1994", Astron. Lett., 23, 818-826. [ADS] (Cited on page 6.)

Kramar, M., Jones, S., Davila, J., Inhester, B. and Mierla, M., 2009, "On the Tomographic Reconstruction of the 3D Electron Density for the Solar Corona from STEREO COR1 Data", Solar Phys., 259, 109-121. [DOI], [ADS] (Cited on pages 15 and 32.)

Krucker, S., Saint-Hilaire, P., Christe, S., White, S.M., Chavier, A.D., Bale, S.D. and Lin, R.P., 2008, "Coronal Hard X-Ray Emission Associated with Radio Type III Bursts", Astrophys. J., 681, 644-649. [DOI], [ADS] (Cited on page 65.) 
Krucker, S., Hudson, H.S., Glesener, L., White, S.M., Masuda, S., Wülser, J.-P. and Lin, R.P., 2010, "Measurements of the Coronal Acceleration Region of a Solar Flare", Astrophys. J., 714, 1108-1119. [DOI], [ADS] (Cited on page 66.)

Kumar, P., Srivastava, A.K., Filippov, B. and Uddin, W., 2010, "Multiwavelength Study of the M8.9/3B Solar Flare from AR NOAA 10960", Solar Phys., 266, 39-58. [DOI], [ADS], [arXiv:1005.5244 [astro-ph.SR]] (Cited on page 66.)

Kwon, R.-Y., Chae, J. and Zhang, J., 2010, "Stereoscopic Determination of Heights of Extreme Ultraviolet Bright Points Using Data Taken by SECCHI/EUVI Aboard STEREO", Astrophys. J., 714, 130-137. [DOI], [ADS] (Cited on page 62.)

Labrosse, N., Dalla, S. and Marshall, S., 2010a, "Automatic Detection of Limb Prominences in 304 Å EUV Images", Solar Phys., 262, 449-460. [DOI], [ADS], [arXiv:0912.1099 [astro-ph.SR]] (Cited on page 58.)

Labrosse, N., Heinzel, P., Vial, J.-C., Kucera, T., Parenti, S., Gunár, S., Schmieder, B. and Kilper, G., 2010b, "Physics of Solar Prominences: I. Spectral Diagnostics and Non-LTE Modelling", Space Sci. Rev., 151, 243-332. [DOI], [ADS], [arXiv:1001.1620 [astro-ph.SR]] (Cited on page 58.)

Landi, E., Miralles, M.P., Curdt, W. and Hara, H., 2009, "Physical Properties of Cooling Plasma in Quiescent Active Region Loops", Astrophys. J., 695, 221-237. [DOI], [ADS] (Cited on page 43.)

Landi, E., Raymond, J.C., Miralles, M.P. and Hara, H., 2010, "Physical Conditions in a Coronal Mass Ejection from Hinode, Stereo, and SOHO Observations", Astrophys. J., 711, 75-98. [DOI], [ADS] (Cited on page 69.)

Lee, J., White, S.M., Kundu, M.R., Mikić, Z. and McClymont, A.N., 1999, "A Test for Coronal Magnetic Field Extrapolations", Astrophys. J., 510, 413-421. [DOI], [ADS] (Cited on page 22.)

Lenz, D.D., Deluca, E.E., Golub, L., Rosner, R. and Bookbinder, J.A., 1999, "Temperature and Emission-Measure Profiles along Long-lived Solar Coronal Loops Observed with the Transition Region and Coronal Explorer", Astrophys. J. Lett., 517, L155-L158. [DOI], [ADS], [arXiv:astroph/9903491] (Cited on page 43.)

Li, T., Zhang, J., Zhao, H. and Yang, S., 2010a, "Three-dimensional Shape and Evolution of Two Eruptive Filaments", Astrophys. J., 720, 144-149. [DOI], [ADS] (Cited on pages 59 and 61.)

Li, Y., Lynch, B.J., Stenborg, G., Luhmann, J.G., Huttunen, K.E.J., Welsch, B.T., Liewer, P.C. and Vourlidas, A., 2008, "The Solar Magnetic Field and Coronal Dynamics of the Eruption on 2007 May 19", Astrophys. J. Lett., 681, L37-L40. [DOI], [ADS] (Cited on page 59.)

Li, Y., Lynch, B.J., Welsch, B.T., Stenborg, G.A., Luhmann, J.G., Fisher, G.H., Liu, Y. and Nightingale, R.W., 2010b, "Sequential Coronal Mass Ejections from AR8038 in May 1997", Solar Phys., 264, 149-164. [DOI], [ADS] (Cited on page 71.)

Liewer, P.C., Davis, J.M., de Jong, E.M., Gary, G.A., Klimchuk, J.A. and Reinert, R.P., 1998, "Report on new mission concept study: Stereo X-Ray Corona Imager mission", in Missions to the Sun II, San Diego, CA, USA, 22 July 1998, (Ed.) Korendyke, C.M., vol. 3442 of Proc. SPIE, pp. 53-64, SPIE, Bellingham, WA. [DOI], [ADS] (Cited on page 8.)

Liewer, P.C., Hall, J.R., De Jong, M., Socker, D.G., Howard, R.A., Crane, P.C., Reiser, P., Rich, N. and Vourlidas, A., 2001, "Determination of three-dimensional structure of coronal streamers and relationship to the solar magnetic field", J. Geophys. Res., 106, 15,903-15,916. [DOI], [ADS] (Cited on page 31.)

Living Reviews in Solar Physics

http://www. livingreviews.org/lrsp-2011-5 
Liewer, P.C., Luhmann, J.G., Huttunen, E., Li, Y., Howard, R.A., Thompson, W.T., Dejong, E.M., Hall, J.R., Falconer, D.A. and Vourlidas, A., 2008, "Stereoscopic Analysis of STEREO/EUVI Observations of May 19, 2007 Erupting Filament", 37th COSPAR Scientific Assembly, Montréal, Canada, 13-20 July 2008, conference paper. [ADS] (Cited on page 59.)

Liewer, P.C., de Jong, E.M., Hall, J.R., Howard, R.A., Thompson, W.T., Culhane, J.L., Bone, L. and van Driel-Gesztelyi, L., 2009, "Stereoscopic Analysis of the 19 May 2007 Erupting Filament", Solar Phys., 256, 57-72. [DOI], [ADS], [arXiv:0904.1055 [astro-ph.SR]] (Cited on pages 22, 59, and 60.)

Long, D.M., Gallagher, P.T., McAteer, R.T.J. and Bloomfield, D.S., 2008, "The Kinematics of a Globally Propagating Disturbance in the Solar Corona", Astrophys. J. Lett., 680, L81-L84. [DOI], [ADS], [arXiv:0805.2023] (Cited on page 71.)

Luhmann, J.G., Curtis, D.W., Schroeder, P., McCauley, J., Lin, R.P., Larson, D.E., Bale, S.D., Sauvaud, J.-A., Aoustin, C., Mewaldt, R.A., Cummings, A.C., Stone, E.C., Davis, A.J., Cook, W.R., Kecman, B., Wiedenbeck, M.E., von Rosenvinge, T., Acuna, M.H., Reichenthal, L.S., Shuman, S., Wortman, K.A., Reames, D.V., Mueller-Mellin, R., Kunow, H., Mason, G.M., Walpole, P., Korth, A., Sanderson, T.R., Russell, C.T. and Gosling, J.T., 2008, "STEREO IMPACT Investigation Goals, Measurements, and Data Products Overview", Space Sci. Rev., 136, 117-184. [DOI], [ADS] (Cited on page 8.)

Lundquist, L.L., Fisher, G.H. and McTiernan, J.M., 2008a, "Forward Modeling of Active Region Coronal Emissions. I. Methods and Testing", Astrophys. J. Suppl. Ser., 179, 509-533. [DOI], [ADS] (Cited on page 26.)

Lundquist, L.L., Fisher, G.H., Metcalf, T.R., Leka, K.D. and McTiernan, J.M., 2008b, "Forward Modeling of Active Region Coronal Emissions. II. Implications for Coronal Heating", Astrophys. J., 689, 1388-1405. [DOI], [ADS] (Cited on page 26.)

Lynch, B.J., Li, Y., Thernisien, A.F.R., Robbrecht, E., Fisher, G.H., Luhmann, J.G. and Vourlidas, A., 2010, "Sun to $1 \mathrm{AU}$ propagation and evolution of a slow streamer-blowout coronal mass ejection", J. Geophys. Res., 115, A07106. [DOI], [ADS] (Cited on page 32.)

Ma, S., Wills-Davey, M.J., Lin, J., Chen, P.F., Attrill, G.D.R., Chen, H., Zhao, S., Li, Q. and Golub, L., 2009, "A New View of Coronal Waves from STEREO", Astrophys. J., 707, 503-509. [DOI], [ADS] (Cited on page 72.)

Ma, S., Attrill, G.D.R., Golub, L. and Lin, J., 2010, "Statistical Study of Coronal Mass Ejections With and Without Distinct Low Coronal Signatures", Astrophys. J., 722, 289-301. [DOI], [ADS] (Cited on page 67.)

Madjarska, M.S., 2011, "Dynamics and plasma properties of an X-ray jet from SUMER, EIS, XRT, and EUVI A and B simultaneous observations", Astron. Astrophys., 526, A19. [DOI], [ADS], [arXiv:1008.3983 [astro-ph.SR]] (Cited on page 62.)

Marsh, M.S. and Walsh, R.W., 2009, "Using HINODE/Extreme-Ultraviolet Imaging Spectrometer to Confirm a Seismologically Inferred Coronal Temperature", Astrophys. J. Lett., 706, L76-L79. [DOI], [ADS], [arXiv:0910.3823 [astro-ph.SR]] (Cited on page 57.)

Marsh, M.S., Walsh, R.W. and Plunkett, S., 2009, "Three-dimensional Coronal Slow Modes: Toward Three-dimensional Seismology", Astrophys. J., 697, 1674-1680. [DOI], [ADS], [arXiv:0903.4039 [astro-ph.SR]] (Cited on pages 57 and 58.) 
Matthews, S.A. and Culhane, J.L., 2007, "Magnetic coupling of the Sun-Earth system - The view from STEREO", Adv. Space Res., 39, 1791-1803. [DOI], [ADS] (Cited on page 5.)

McIntosh, S.W., Innes, D.E., de Pontieu, B. and Leamon, R.J., 2010, "STEREO observations of quasi-periodically driven high velocity outflows in polar plumes", Astron. Astrophys., 510, L2. [DOI], [ADS], [arXiv:1001.3377 [astro-ph.SR]] (Cited on page 62.)

Metcalf, T.R., Jiao, L., McClymont, A.N., Canfield, R.C. and Uitenbroek, H., 1995, "Is the solar chromospheric magnetic field force-free?", Astrophys. J., 439, 474-481. [DOI], [ADS] (Cited on page 25.)

Michels, D.J., 2002, "The SECCHI Solar Plasma Imager for STEREO", in Space Weather Study Using Multipoint Techniques, Proceedings of the COSPAR Colloquium held in Pacific Green Bay, Wanli, Taipei, Taiwan, 27 - 29 September 2000, (Ed.) Lyu, L.-H., vol. 12 of COSPAR Colloquia Series, pp. 49-54, Pergamon, Oxford. [ADS] (Cited on page 8.)

Mierla, M., Inhester, B., Antunes, A., Boursier, Y., Byrne, J.P., Colaninno, R., Davila, J., de Koning, C.A., Gallagher, P.T., Gissot, S., Howard, R.A., Howard, T.A., Kramar, M., Lamy, P., Liewer, P.C., Maloney, S., Marqué, C., McAteer, R.T.J., Moran, T., Rodriguez, L., Srivastava, N., St. Cyr, O.C., Stenborg, G., Temmer, M., Thernisien, A., Vourlidas, A., West, M.J., Wood, B.E. and Zhukov, A.N., 2010, "On the 3-D reconstruction of Coronal Mass Ejections using coronagraph data", Ann. Geophys., 28, 203-215. [DOI], [ADS] (Cited on page 5.)

Milligan, R.O., McAteer, R.T.J., Dennis, B.R. and Young, C.A., 2010, "Evidence of a PlasmoidLooptop Interaction and Magnetic Inflows During a Solar Flare/Coronal Mass Ejection Eruptive Event", Astrophys. J., 713, 1292-1300. [DOI], [ADS], [arXiv:1003.0665 [astro-ph.SR]] (Cited on page 66.)

Minnaert, M., 1930, "On the continuous spectrum of the corona and its polarisation", Z. Astrophys., 1, 209-236. [ADS] (Cited on page 14.)

Mok, Y., Mikić, Z., Lionello, R. and Linker, J.A., 2005, "Calculating the Thermal Structure of Solar Active Regions in Three Dimensions", Astrophys. J., 621, 1098-1108. [DOI], [ADS] (Cited on page 26.)

Moore, R.L., Cirtain, J.W., Sterling, A.C. and Falconer, D.A., 2010, "Dichotomy of Solar Coronal Jets: Standard Jets and Blowout Jets", Astrophys. J., 720, 757-770. [DOI], [ADS] (Cited on pages 62 and 63.)

Moran, T.G. and Davila, J.M., 2004, "Three-Dimensional Polarimetric Imaging of Coronal Mass Ejections", Science, 305, 66-71. [DOI], [ADS] (Cited on page 7.)

Moran, T.G., Davila, J.M. and Thompson, W.T., 2010, "Three-Dimensional Polarimetric Coronal Mass Ejection Localization Tested Through Triangulation", Astrophys. J., 712, 453-458. [DOI], [ADS] (Cited on page 7.)

Morgan, H. and Habbal, S.R., 2010, "Observational Aspects of the Three-dimensional Coronal Structure Over a Solar Activity Cycle", Astrophys. J., 710, 1-15. [DOI], [ADS] (Cited on pages 15,31 , and 32.)

Morgan, H., Habbal, S.R. and Lugaz, N., 2009, "Mapping the Structure of the Corona Using Fourier Backprojection Tomography", Astrophys. J., 690, 1119-1129. [DOI], [ADS] (Cited on pages 15,31 , and 32. ) 
Mueller, J.T., Maldonado, H. and Driesman, A.S., 2003, "STEREO: The challenges", Acta Astronaut., 53, 729-738. [DOI], [ADS] (Cited on page 8.)

Nisticò, G., Bothmer, V., Patsourakos, S. and Zimbardo, G., 2009, "Characteristics of EUV Coronal Jets Observed with STEREO/SECCHI", Solar Phys., 259, 87-108. [DOI], [ADS], [arXiv:0906.4407 [astro-ph.SR]] (Cited on page 62.)

Nitta, N., Van Driel-Gesztelyi, L. and Harra-Murnion, L.K., 1999, "Flare loop geometry", Solar Phys., 189, 181-198. [DOI], [ADS] (Cited on pages 26 and 65.)

Noglik, J.B., Walsh, R.W., Maclean, R.C. and Marsh, M.S., 2009, "Deconstructing Active Region AR10961 Using STEREO, Hinode, TRACE, and SOHO”, Astrophys. J., 703, 1923-1938. [DOI], [ADS], [arXiv:0807.4487] (Cited on page 35.)

Ofman, L., 2009, "Progress, Challenges, and Perspectives of the 3D MHD Numerical Modeling of Oscillations in the Solar Corona", Space Sci. Rev., 149, 153-174. [DOI], [ADS] (Cited on page 54.)

Paraschiv, A.R., Lacatus, D.A., Badescu, T., Lupu, M.G., Simon, S., Sandu, S.G., Mierla, M. and Rusu, M.V., 2010, "Study of Coronal Jets During Solar Minimum Based on STEREO/SECCHI Observations", Solar Phys., 264, 365-375. [DOI], [ADS] (Cited on page 62.)

Pariat, E., Antiochos, S.K. and DeVore, C.R., 2010, "Three-dimensional Modeling of Quasihomologous Solar Jets", Astrophys. J., 714, 1762-1778. [DOI], [ADS] (Cited on pages 62 and 64.)

Patsourakos, S. and Vourlidas, A., 2009, “"Extreme Ultraviolet Waves' are Waves: First Quadrature Observations of an Extreme Ultraviolet Wave from STEREO", Astrophys. J. Lett., 700, L182L186. [DOI], [ADS], [arXiv:0905.2164 [astro-ph.SR]] (Cited on page 72.)

Patsourakos, S., Pariat, E., Vourlidas, A., Antiochos, S.K. and Wülser, J.-P., 2008, "STEREO SECCHI Stereoscopic Observations Constraining the Initiation of Polar Coronal Jets", Astrophys. J. Lett., 680, L73-L76. [DOI], [ADS], [arXiv:0804.4862] (Cited on page 62.)

Patsourakos, S., Vourlidas, A., Wang, Y.M., Stenborg, G. and Thernisien, A., 2009, "What Is the Nature of EUV Waves? First STEREO 3D Observations and Comparison with Theoretical Models", Solar Phys., 259, 49-71. [DOI], [ADS], [arXiv:0905.2189 [astro-ph.SR]] (Cited on page 72.)

Patsourakos, S., Vourlidas, A. and Kliem, B., 2010, "Toward understanding the early stages of an impulsively accelerated coronal mass ejection. SECCHI observations", Astron. Astrophys., 522, A100. [DOI], [ADS], [arXiv:1008.1171 [astro-ph.SR]] (Cited on page 68.)

Petrie, G.J.D., Canou, A. and Amari, T., 2011, "Nonlinear Force-Free and Potential-Field Models of Active-Region and Global Coronal Fields during the Whole Heliosphere Interval", Solar Phys., in press. [DOI], [ADS], [arXiv:1010.6283 [astro-ph.SR]] (Cited on page 22.)

Portier-Fozzani, F. and Inhester, B., 2001, "3D Coronal structures and their evolutions measured by Stereoscopy, consequences for Space Weather and the STEREO mission", Space Sci. Rev., 97, 51-54. [DOI], [ADS] (Cited on page 38.)

Portier-Fozzani, F., Aschwanden, M., Démoulin, P., Neupert, W. and Delaboudinière, J.-P. [EIT Team], 2001, "Measurement of coronal magnetic twists during loop emergence of NOAA 8069", Solar Phys., 203, 289-308. [DOI], [ADS] (Cited on page 38.)

Quémerais, E. and Lamy, P., 2002, "Two-dimensional electron density in the solar corona from inversion of white light images - Application to SOHO/LASCO-C2 observations", Astron. Astrophys., 393, 295-304. [DOI], [ADS] (Cited on pages 7 and 29.) 
Raftery, C.L., Gallagher, P.T., McAteer, R.T.J., Lin, C.-H. and Delahunt, G., 2010, "Evidence for Internal Tether-cutting in a Flare/Coronal Mass Ejection Observed by MESSENGER, RHESSI, and STEREO", Astrophys. J., 721, 1579-1584. [DOI], [ADS] (Cited on page 66.)

Raouafi, N.-E., 2009, "Observational Evidence for Coronal Twisted Flux Rope", Astrophys. J. Lett., 691, L128-L132. [DOI], [ADS], [arXiv:0901.0045 [astro-ph.SR]] (Cited on page 58.)

Raouafi, N.-E., Petrie, G.J.D., Norton, A.A., Henney, C.J. and Solanki, S.K., 2008, "Evidence for Polar Jets as Precursors of Polar Plume Formation", Astrophys. J. Lett., 682, L137-L140. [DOI], [ADS], [arXiv:0806.3045] (Cited on page 62.)

Robbrecht, E. and Wang, Y.-M., 2010, "The Temperature-dependent Nature of Coronal Dimmings", Astrophys. J. Lett., 720, L88-L92. [DOI], [ADS], [arXiv:1007.5191 [astro-ph.SR]] (Cited on page 69.)

Robbrecht, E., Patsourakos, S. and Vourlidas, A., 2009, "No Trace Left Behind: STEREO Observation of a Coronal Mass Ejection Without Low Coronal Signatures", Astrophys. J., 701, 283-291. [DOI], [ADS], [arXiv:0905.2583 [astro-ph.SR]] (Cited on page 67.)

Rodriguez, L., Zhukov, A.N., Gissot, S. and Mierla, M., 2009, "Three-Dimensional Reconstruction of Active Regions", Solar Phys., 256, 41-55. [DOI], [ADS] (Cited on pages 33 and 35.)

Ruan, P., Wiegelmann, T., Inhester, B., Neukirch, T., Solanki, S.K. and Feng, L., 2008, "A first step in reconstructing the solar corona self-consistently with a magnetohydrostatic model during solar activity minimum", Astron. Astrophys., 481, 827-834. [DOI], [ADS] (Cited on page 22.)

Rust, D.M., 1998, "The Solar STEREO Mission", in A Crossroads for European Solar and Heliospheric Physics: Recent Achievements and Future Mission Possibilities, Proceedings of a conference held at Puerto de la Cruz, Tenerife, Canary Islands, Spain, March 23-27, 1998, (Eds.) Priest, E.R., Moreno-Insertis, F., Harris, R.A., vol. 417 of ESA Conference Proceedings, pp. 133-143, ESA Publications Division, Noordwijk. [ADS] (Cited on page 8.)

Ryabov, B.I., Maksimov, V.P., Lesovoi, S.V., Shibasaki, K., Nindos, A. and Pevtsov, A., 2005, "Coronal Magnetography of Solar Active Region 8365 with the SSRT and NoRH Radio Heliographs", Solar Phys., 226, 223-237. [DOI], [ADS] (Cited on page 22.)

Saez, F., Zhukov, A.N., Lamy, P. and Llebaria, A., 2005, "On the 3-dimensional structure of the streamer belt of the solar corona", Astron. Astrophys., 442, 351-358. [DOI], [ADS] (Cited on pages 31 and 32.)

Saez, F., Llebaria, A., Lamy, P. and Vibert, D., 2007, "Three-dimensional reconstruction of the streamer belt and other large-scale structures of the solar corona. I. Method", Astron. Astrophys., 473, 265-277. [DOI], [ADS] (Cited on page 29.)

Sandman, A.W. and Aschwanden, M.J., 2011, "A new method for modeling the coronal magnetic field with STEREO and submerged dipoles", Solar Phys., 270, 503-522. [DOI], [ADS] (Cited on pages 22 and 45.)

Sandman, A.W., Aschwanden, M.J., DeRosa, M.L., Wülser, J.P. and Alexander, D., 2009, "Comparison of STEREO/EUVI Loops with Potential Magnetic Field Models", Solar Phys., 259, 1-11. [DOI], [ADS] (Cited on pages 22, 45, and 46.)

Schmidt, J.M. and Ofman, L., 2010, "Global Simulation of an Extreme Ultraviolet Imaging Telescope Wave", Astrophys. J., 713, 1008-1015. [DOI], [ADS] (Cited on page 72.) 
Schmidt, W.K.H. and Bothmer, V., 1996, "Stereoscopic viewing of solar coronal and interplanetary activity", Adv. Space Res., 17, 369-376. [DOI], [ADS] (Cited on pages 5 and 8.)

Schrijver, C.J., 2009, "Driving major solar flares and eruptions: A review", Adv. Space Res., 43, 739-755. [DOI], [ADS], [arXiv:0811.0787] (Cited on page 67.)

Schrijver, C.J. and Title, A.M., 2011, "Long-range magnetic couplings between solar flares and coronal mass ejections observed by SDO and STEREO", J. Geophys. Res., 116, A04108. [DOI] (Cited on page 22.)

Schrijver, C.J., Sandman, A.W., Aschwanden, M.J. and DeRosa, M.L., 2004, "The Coronal Heating Mechanism as Identified by Full-Sun Visualizations", Astrophys. J., 615, 512-525. [DOI], [ADS] (Cited on page 26.)

Seaton, D.B., Mierla, M., Berghmans, D., Zhukov, A.N. and Dolla, L., 2011, "SWAP-SECCHI Observations of a Mass-loading Type Solar Eruption", Astrophys. J. Lett., 727, L10. [DOI], [ADS], [arXiv:1011.1144 [astro-ph.SR]] (Cited on page 59.)

Sheeley Jr, N.R. and Rouillard, A.P., 2010, "Tracking Streamer Blobs into the Heliosphere", Astrophys. J., 715, 300-309. [DOI], [ADS], [arXiv:1006.5379 [astro-ph.SR]] (Cited on page 32.)

Sheeley Jr, N.R., Lee, D.D.-H., Casto, K.P., Wang, Y.-M. and Rich, N.B., 2009, "The Structure of Streamer Blobs", Astrophys. J., 694, 1471-1480. [DOI], [ADS] (Cited on page 32.)

Socker, D.G., 1998, "Stereographic image potential of a STEREO mission", in Missions to the Sun II, San Diego, CA, USA, 22 July 1998, (Ed.) Korendyke, C.M., vol. 3442 of Proc. SPIE, pp. 44-52, SPIE, Bellingham, WA. [DOI], [ADS] (Cited on page 8.)

Socker, D.G., Antiochos, S.K., Brueckner, G.E., Cook, J.W., Dere, K.P., Howard, R.A., Karpen, J.T., Klimchuk, J.A., Korendyke, C.M., Michels, D.J., Moses, J.D., Prinz, D.K., Sheeley Jr, N.R., Wu, S.T., Buffington, A., Jackson, B.V., Labonte, B., Lamy, P.L., Rosenbauer, H., Schwenn, R., Burlaga, L., Davila, J.M., Davis, J.M., Goldstein, B., Harris, H., Liewer, P.C., Neugebauer, M., Hildner, E., Pizzo, V.J., Moulton, N.E., Linker, J.A. and Mikić, Z., 1996, "STEREO: a solar terrestrial event observer mission concept", in Missions to the Sun, Denver, CO, USA, 8 August 1996, (Ed.) Rust, D.M., vol. 2804 of Proc. SPIE, pp. 50-61, SPIE, Bellingham, WA. [DOI], [ADS] (Cited on page 8.)

Socker, D.G., Howard, R.A., Korendyke, C.M., Simnett, G.M. and Webb, D.F., 2000, "NASA Solar Terrestrial Relations Observatory (STEREO) mission heliospheric imager", in Instrumentation for UV/EUV Astronomy and Solar Missions, San Diego, CA, USA, 30 July 2000, (Eds.) Fineschi, S., Korendyke, C.N., Siegmund, O.H., Woodgate, B.E., vol. 4139 of Proc. SPIE, pp. 284-293, SPIE, Bellingham, WA. [DOI], [ADS] (Cited on page 8.)

Srivastava, A.K., Zaqarashvili, T.V., Kumar, P. and Khodachenko, M.L., 2010, "Observation of Kink Instability During Small B5.0 Solar Flare on 2007 June 4", Astrophys. J., 715, 292-299. [DOI], [ADS], [arXiv:1004.1454 [astro-ph.SR]] (Cited on page 54.)

Srivastava, N., Mierla, M. and Rodriguez, L., 2011, "On three-dimensional aspects of CMEs, their source regions and interplanetary manifestations: Introduction to special issue", J. Atmos. Sol.Terr. Phys., 73, 1077-1081. [DOI], [ADS] (Cited on page 5.)

Staedter, T., 2006, "Twin Spacecraft to Provide Three-Dimensional View of Sun", Space Weather, 4, S08 006. [DOI], [ADS] (Cited on page 5.) 
Sterling, A.C., Chifor, C., Mason, H.E., Moore, R.L. and Young, P.R., 2010, "Evidence for magnetic flux cancelation leading to an ejective solar eruption observed by Hinode, TRACE, STEREO, and SoHO/MDI", Astron. Astrophys., 521, A49. [DOI], [ADS] (Cited on page 59.)

Su, Y., van Ballegooijen, A., Schmieder, B., Berlicki, A., Guo, Y., Golub, L. and Huang, G., 2009, "Flare Energy Build-up in a Decaying Active Region Near a Coronal Hole", Astrophys. J., 704, 341-353. [DOI], [ADS] (Cited on page 66.)

Su, Y., van Ballegooijen, A. and Golub, L., 2010, "Structure and Dynamics of Quiescent Filament Channels Observed by Hinode/XRT and STEREO/EUVI", Astrophys. J., 721, 901-910. [DOI], [ADS] (Cited on page 58.)

Tappin, S.J. and Howard, T.A., 2009, "Interplanetary Coronal Mass Ejections Observed in the Heliosphere: 2. Model and Data Comparison", Space Sci. Rev., 147, 55-87. [DOI], [ADS] (Cited on page 5.)

Temmer, M., Veronig, A.M., Kontar, E.P., Krucker, S. and Vršnak, B., 2010, "Combined STEREO/RHESSI Study of Coronal Mass Ejection Acceleration and Particle Acceleration in Solar Flares", Astrophys. J., 712, 1410-1420. [DOI], [ADS], [arXiv:1002.3080 [astro-ph.SR]] (Cited on page 66.)

Thompson, W.T., 2006, "Coordinate systems for solar image data", Astron. Astrophys., 449, 791-803. [DOI], [ADS] (Cited on page 8.)

Thompson, W.T., 2011, "Strong rotation of an erupting quiescent polar crown prominence", J. Atmos. Sol.-Terr. Phys., 73, 1138-1147. [DOI], [ADS] (Cited on page 22.)

Thompson, W.T. and Reginald, N.L., 2008, "The Radiometric and Pointing Calibration of SECCHI COR1 on STEREO", Solar Phys., 250, 443-454. [DOI], [ADS] (Cited on page 8.)

Thompson, W.T. and Wei, K., 2010, "Use of the FITS World Coordinate System by STEREO/SECCHI", Solar Phys., 261, 215-222. [DOI], [ADS] (Cited on page 8.)

Thompson, W.T., Davila, J.M., Fisher, R.R., Orwig, L.E., Mentzell, J.E., Hetherington, S.E., Derro, R.J., Federline, R.E., Clark, D.C., Chen, P.T.C., Tveekrem, J.L., Martino, A.J., Novello, J., Wesenberg, R.P., St Cyr, O.C., Reginald, N.L., Howard, R.A., Mehalick, K.I., Hersh, M.J., Newman, M.D., Thomas, D.L., Card, G.L. and Elmore, D.F., 2003, "COR1 inner coronagraph for STEREO-SECCHI", in Innovative Telescopes and Instrumentation for Solar Astrophysics, Waikoloa, HI, USA, 24 August 2002, (Eds.) Keil, S.L., Avakyan, S.V., vol. 4853 of Proc. SPIE, pp. 1-11, SPIE, Bellingham, WA. [DOI], [ADS] (Cited on page 8.)

Thompson, W.T., Wei, K., Burkepile, J.T., Davila, J.M. and St Cyr, O.C., 2010, "Background Subtraction for the SECCHI/COR1 Telescope Aboard STEREO", Solar Phys., 262, 213-231. [DOI], [ADS] (Cited on page 8.)

Tian, H., Marsch, E., Curdt, W. and He, J., 2009, "Upflows in Funnel-like Legs of Coronal Magnetic Loops", Astrophys. J., 704, 883-890. [DOI], [ADS], [arXiv:0909.0739 [astro-ph.SR]] (Cited on page 43.)

van de Hulst, H.C., 1950, "The electron density of the solar corona", Bull. Astron. Inst. Neth., XI, 135-150. [ADS] (Cited on page 14.)

Vásquez, A.M., Frazin, R.A. and Kamalabadi, F., 2009, "3D Temperatures and Densities of the Solar Corona via Multi-Spacecraft EUV Tomography: Analysis of Prominence Cavities", Solar Phys., 256, 73-85. [DOI], [ADS] (Cited on page 29.) 
Vásquez, A.M., Frazin, R.A. and Manchester IV, W.B., 2010, "The Solar Minimum Corona from Differential Emission Measure Tomography", Astrophys. J., 715, 1352-1365. [DOI], [ADS] (Cited on pages 29 and 30.)

Vásquez, A.M., Huang, Z., Manchester IV, W.B. and Frazin, R.A., 2011, "The WHI Corona from Differential Emission Measure Tomography", Solar Phys., in press. [DOI], [ADS], [arXiv:1012.5953] (Cited on pages 29 and 31.)

Vedenov, A.A., Koutvitsky, V.A., Koutchmy, S., Molodensky, M.M. and Oraevsky, V.N., 2000, "On Stereoimages of Some Structures in the Solar Atmosphere", Astron. Rep., 44, 112-121. [DOI], [ADS] (Cited on page 6.)

Veronig, A.M., Temmer, M. and Vršnak, B., 2008, "High-Cadence Observations of a Global Coronal Wave by STEREO EUVI", Astrophys. J. Lett., 681, L113-L116. [DOI], [ADS], [arXiv:0806.0710] (Cited on page 72.)

Veronig, A.M., Muhr, N., Kienreich, I.W., Temmer, M. and Vršnak, B., 2010, "First Observations of a Dome-shaped Large-scale Coronal Extreme-ultraviolet Wave", Astrophys. J. Lett., 716, L57-L62. [DOI], [ADS], [arXiv:1005.2060 [astro-ph.SR]] (Cited on pages 72 and 73.)

Verwichte, E., Aschwanden, M.J., Van Doorsselaere, T., Foullon, C. and Nakariakov, V.M., 2009, "Seismology of a Large Solar Coronal Loop from EUVI/STEREO Observations of its Transverse Oscillation", Astrophys. J., 698, 397-404. [DOI], [ADS] (Cited on pages 48, 49, 50, and 52.)

Vourlidas, A. and Howard, R.A., 2006, "The Proper Treatment of Coronal Mass Ejection Brightness: A New Methodology and Implications for Observations", Astrophys. J., 642, 1216-1221. [DOI], [ADS] (Cited on pages 15 and 16.)

Vourlidas, A., Howard, R.A., Esfandiari, E., Patsourakos, S., Yashiro, S. and Michalek, G., 2010, "Comprehensive Analysis of Coronal Mass Ejection Mass and Energy Properties Over a Full Solar Cycle", Astrophys. J., 722, 1522-1538. [DOI], [ADS], [arXiv:1008.3737 [astro-ph.SR]] (Cited on page 69.)

Wang, Y., Cao, H., Chen, J., Zhang, T., Yu, S., Zheng, H., Shen, C., Zhang, J. and Wang, S., 2010, "Solar Limb Prominence Catcher and Tracker (SLIPCAT): An Automated System and its Preliminary Statistical Results", Astrophys. J., 717, 973-986. [DOI], [ADS], [arXiv:1004.4553 [astro-ph.SR]] (Cited on page 58.)

Warren, H.P., Kim, D.M., DeGiorgi, A.M. and Ugarte-Urra, I., 2010, "Modeling Evolving Coronal Loops with Observations from STEREO, Hinode, and TRACE", Astrophys. J., 713, 1095-1107. [DOI], [ADS], [arXiv:0904.3920 [astro-ph.SR]] (Cited on page 43.)

Wiegelmann, T. and Inhester, B., 2003, "Magnetic modeling and tomography: First steps towards a consistent reconstruction of the solar corona", Solar Phys., 214, 287-312. [DOI], [ADS], [arXiv:0802.0687] (Cited on page 22.)

Wiegelmann, T. and Inhester, B., 2006, "Magnetic Stereoscopy", Solar Phys., 236, 25-40. [DOI], [ADS], [arXiv:astro-ph/0612636] (Cited on pages 22 and 44.)

Wiegelmann, T. and Neukirch, T., 2002, "Including stereoscopic information in the reconstruction of coronal magnetic fields", Solar Phys., 208, 233-251. [DOI], [ADS], [arXiv:0801.3234] (Cited on pages 22 and 44.)

Wiegelmann, T., Inhester, B. and Feng, L., 2009, "Solar stereoscopy - where are we and what developments do we require to progress?", Ann. Geophys., 27, 2925-2936. [DOI], [ADS], [arXiv:0912.1267 [astro-ph.SR]] (Cited on pages 5, 22, and 44.) 
Wülser, J.-P., Lemen, J.R., Tarbell, T.D., Wolfson, C.J., Cannon, J.C., Carpenter, B.A., Duncan, D.W., Gradwohl, G.S., Meyer, S.B., Moore, A.S., Navarro, R.L., Pearson, J.D., Rossi, G.R., Springer, L.A., Howard, R.A., Moses, J.D., Newmark, J.S., Delaboudinière, J.-P., Artzner, G.E., Auchère, F., Bougnet, M., Bouyries, P., Bridou, F., Clotaire, J.-Y., Colas, G., Delmotte, F., Jerome, A., Lamare, M., Mercier, R., Mullot, M., Ravet, M.-F., Song, X., Bothmer, V. and Deutsch, W., 2004, "EUVI: the STEREO-SECCHI extreme ultraviolet imager", in Telescopes and Instrumentation for Solar Astrophysics, San Diego, CA, USA, 7 August 2003, (Eds.) Fineschi, S., Gummin, M.A., vol. 5171 of Proc. SPIE, pp. 111-122, SPIE, Bellingham, WA. [DOI], [ADS] (Cited on page 8.)

Xu, Y., Jing, J. and Wang, H., 2010, "Measurements of Filament Height in H $\alpha$ and EUV $304 \AA "$, Solar Phys., 264, 81-91. [DOI], [ADS] (Cited on page 59.)

Young, P.R. and Landi, E., 2009, "Chianti - An Atomic Database for Emission Lines. XI. ExtremeUltraviolet Emission Lines of Fe VII, Fe VIII, and Fe IX Observed by Hinode/EIS", Astrophys. J., 707, 173-192. [DOI], [ADS], [arXiv:0907.3488 [astro-ph.SR]] (Cited on page 8.)

Zhang, J. and Li, L.-P., 2009, "Two types of dynamic cool coronal structures observed with STEREO and Hinode", Res. Astron. Astrophys., 9, 1368-1376. [DOI], [ADS], [arXiv:0909.1864 [physics.space-ph]] (Cited on page 43.)

Zheng, R., Jiang, Y.C., Yang, L.H. and Bi, Y., 2011, "The coronal mass ejection associated with the loop eruption and coronal dimmings on 2009 December 13", Astrophys. Space Sci., 332, 81-89. [DOI], [ADS] (Cited on page 71.)

Zhukov, A.N., Rodriguez, L. and de Patoul, J., 2009, "STEREO/SECCHI Observations on 8 December 2007: Evidence Against the Wave Hypothesis of the EIT Wave Origin", Solar Phys., 259, 73-85. [DOI], [ADS] (Cited on page 72.)

Zidowitz, S., 1997, "The Coronal Structure in August 1996-A Tomographic Reconstruction", in The Corona and Solar Wind Near Minimum Activity, Proceedings of the Fifth SOHO Workshop, held at the University of Oslo, Norway, 17-20 June 1997, (Ed.) Wilson, A., vol. 404 of ESA Conference Proceedings, pp. 757-761, ESA Publications Division, Noordwijk. [ADS] (Cited on page 15.)

Zidowitz, S., 1999, "Coronal structure of the Whole Sun Month: A tomographic reconstruction", J. Geophys. Res., 104, 9727-9734. [DOI], [ADS] (Cited on pages 15, 29, and 31.)

Zidowitz, S., Inhester, B. and Epple, A., 1996, "Tomographic inversion of coronagraph images", in Solar Wind Eight, Proceedings of the Eighth International Solar Wind Conference, Dana Point, CA 1995, (Eds.) Winterhalter, D., Gosling, J.T., Habbal, S.R., Kurth, W.S., Neugebauer, M., vol. 382 of AIP Conference Proceedings, pp. 165-168, American Institute of Physics, Woodbury, NY. [DOI], [ADS] (Cited on page 15.) 